\title{
Recent Developments in Mathematical Quantum Chaos
}

\author{
Steve Zelditch
}

\begin{abstract}
This is a survey of recent results on quantum ergodicity, specifically on the large energy limits of matrix elements relative to eigenfunctions of the Laplacian. It is mainly devoted to QUE (quantum unique ergodicity) results, i.e. results on the possible existence of a sparse subsequence of eigenfunctions with anomalous concentration. We cover the lower bounds on entropies of quantum limit measures due to Anantharaman, Nonnenmacher, and Rivière on compact Riemannian manifolds with Anosov flow. These lower bounds give new constraints on the possible quantum limits. We also cover the non-QUE result of Hassell in the case of the Bunimovich stadium. We include some discussion of Hecke eigenfunctions and recent results of Soundararajan completing Lindenstrauss' QUE result, in the context of matrix elements for Fourier integral operators. Finally, in answer to the potential question "why study matrix elements" it presents an application by the author to the geometry of nodal sets.
\end{abstract}

\section{Contents}

1. Wave equation and geodesic flow

2. Weyl law and local Weyl law

3. Invariant states defined by eigenfunctions and their quantum limits

4. Quantum ergodicity and mixing of eigenfunctions

5. Concentration of eigenfunctions around hyperbolic closed geodesics

6. Boundary quantum ergodicity and quantum ergodic restriction

7. Hassell's scarring result for stadia

8. Matrix elements of Fourier integral operators 161

9. QUE of Hecke eigenfunctions

\footnotetext{
Research partially supported by NSF grant DMS 0904252.
} 
10. Variance estimates: Rate of quantum ergodicity and mixing

11. Entropy of quantum limits on manifolds with Anosov geodesic flow

12. Applications to nodal hypersurfaces of eigenfunctions

Quantum chaos on Riemannian manifolds $(M, g)$ is concerned with the asymptotics of eigenvalues and orthonormal bases of eigenfunctions

$$
\Delta \varphi_{j}=\lambda_{j}^{2} \varphi_{j}, \quad\left\langle\varphi_{j}, \varphi_{k}\right\rangle=\delta_{j k}
$$

of the Laplacian $\Delta$ when the geodesic flow $g^{t}: S_{g}^{*} M \rightarrow S_{g}^{*} M$ is ergodic or Anosov or in some other sense chaotic. Model examples include compact or finite volume hyperbolic surfaces $\mathbf{X}_{\Gamma}=\Gamma \backslash \mathbf{H}$, where $\mathbf{H}$ is the hyperbolic plane and $\Gamma \subset P S L(2, \mathbb{R})$ is a discrete subgroup. Of special interest are the arithmetic quotients when $\Gamma$ is a discrete arithmetic subgroup. Other model example include Euclidean domains with ergodic billiards such as the Bunimovich stadium. The general question is, how does the dynamics of the geodesic (or billiard) flow make itself felt in the eigenvalues and eigenfunctions (or more general solutions of the wave equation), which by the correspondence principle of quantum mechanics must reproduce the classical limit as the eigenvalue tends to infinity?

To avoid confusion, we emphasize that we denote eigenvalues of the Laplacian by $\lambda_{j}^{2}$. They are usually viewed as energies $E_{j}=\lambda_{j}^{2}$. Their square roots $\lambda_{j}$ are called the frequencies.

The most basic quantities testing the asymptotics of eigenfunctions are the matrix elements $\left\langle A \varphi_{j}, \varphi_{j}\right\rangle$ of pseudo-differential operators relative to the orthonormal basis of eigenfunctions. As explained below, these matrix elements measure the expected value of the observable $A$ in the energy state $\varphi_{j}$. Much of the work in quantum ergodicity since the pioneering work of A. I. Schnirelman $[\mathbf{S h . 1}]$ is devoted to the study of the limits of $\left\langle A \varphi_{j}, \varphi_{j}\right\rangle$ as $\lambda_{j} \rightarrow \infty$. Although difficult to determine, these limits are still the most accessible aspects of eigenfunctions. A sequence of eigenfunctions is called ergodic or diffuse if the limit tends to $\int_{S_{g}^{*} M} \sigma_{A} d \mu_{L}$ where $\sigma_{A}$ is the prinicipal symbol of $A$ and $d \mu_{L}$ is the Liouville measure.

The first priority of this survey is to cover the important new results on the so-called scarring or QUE (quantum unique ergodicity) problem. Roughly speaking, the problem is whether every orthonormal basis becomes equidistributed in phase space with respect to Liouville measure as the eigenvalue tends to infinity, i.e.

$$
\left\langle A \varphi_{j}, \varphi_{j}\right\rangle \rightarrow \int_{S_{g}^{*} M} \sigma_{A} d \mu_{L}
$$


or whether there exists a sparse exceptional sequence which has a singular concentration. One series of positive results due to N. Anantharaman $[\mathbf{A}]$, N. Anantharaman-S. Nonnenmacher $[\mathbf{A N}]$ (see also $[\mathbf{A N K}]$ ), and G. Rivière [Riv] flows from the study of entropies of quantum limit measures and are based on a difficult and complex microlocal PDE analysis of the long time behavior of the wave group on manifolds with Anosov geodesic flow. These results give lower bounds on entropies of quantum limit measures which imply that the limit measures must be to some extent diffuse. Another series of results due R. Soundararajan [Sound1] use $L$-function methods to complete the QUE result of E. Lindenstrauss [LIND] for Hecke eigenfunctions in the arithmetic case. In the negative direction, A. Hassell [Has] uses microlocal methods to proved the long-standing conjecture that generic stadia are not QUE but rather have exceptional sequences of bouncing ball type modes which concentrate or scar along a Lagrangian submanifold.

QUE is not the only interesting problem in quantum chaos. Another series of results in the arithmetic case are the remarkably sharp Luo-Sarnak asymptotics $[\mathbf{L S}, \mathbf{L S 2}]$ of the variances

$$
\sum_{j: \lambda_{j} \leq \lambda}\left|\left\langle A \varphi_{j}, \varphi_{j}\right\rangle-\int_{S_{g}^{*} M} \sigma_{A} d \mu_{L}\right|^{2}
$$

for holomorphic Hecke eigenforms and their recent generalization to smooth Maass forms by Zhao $[\mathbf{Z h}]$. Physicists (see e.g. $[\mathbf{F P}]$ ) have speculated that the variance is related to the classical auto-correlation function of the geodesic flow. The Luo-Sarnak-Zhao results partially confirm this conjecture in the special case of arithmetic surfaces and Hecke eigenfunctions, but correct it with an extra arithmetic factor.

A final direction we survey is the applications of quantum ergodicity to problems in nodal geometry. Given the large amount of work on matrix elements $\left\langle A \varphi_{j}, \varphi_{j}\right\rangle$, it is natural for geometric or PDE analysts to ask how one can use such matrix elements to study classical problems on eigenfunctions such as growth and distributions of zeros and critical points. One of our themes is that for real analytic $(M, g)$, logarithms $\log \left|\varphi_{j}^{\mathbb{C}}(\zeta)\right|^{2}$ of analytic continuations of ergodic eigenfunctions are asymptotically "maximal plurisubharmonic functions" in Grauert tubes and as a result their zero sets have a special limit distribution [Z5]. Thus, ergodicity causes maximal oscillation in the real and complex domain and gives rise to special distributions of zeros and (conjecturally) to critical points.

In preparing this survey, we were only too aware of the large number of surveys and expository articles that already exist on the QUE and entropy problems, particularly [AN2, ANK, CV3, LIND2, Sar3] (and for earlier work $[\mathbf{Z 1}, \mathbf{Z 2}, \mathbf{M}, \mathbf{S a r}]$ ). To a large degree we closely follow the original sources, emphasizing intuition over the finer technical details. Although we go over much of the same material in what are now standard ways, we also go over some topics that do not seem as well known, and possibly could be 
improved, and we fill in some gaps in the literature. In particular, most expositions emphasize the quantum entropic uncertainty principle of $[\mathbf{A N}]$ due to its structural nature. So instead we emphasize the ideas of $[\mathbf{A}]$, which are less structural but in some ways more geometric.

One of the novelties is the study in $\S 8$ of matrix elements $\left\langle F \varphi_{j}, \varphi_{j}\right\rangle$ of Fourier integral operators $F$ relative to the eigenfunctions. Hecke operators are examples of such $F$ but restrictions to hypersurfaces provide a different kind of $F$ which have recently come up in quantum ergodic restriction theory [TZ3]. We give a rather simple result on quantum limits for Fourier integral operators which answers the question, "what invariance property do quantum limit measures of Hecke eigenfunctions possess?". Hecke analysts have worked with a partial quasi-invariance principle due to Rudnick-Sarnak, and although the exact invariance principle may not simplify the known proofs of QUE it is of interest to know that one exists. We plan to put complete proofs in a future article.

Another aspect of eigenfunctions is their possible concentration of eigenfunctions around closed hyperbolic geodesic. Mass concentration within thin or shrinking tubes around such geodesics has been studied by S. Nonnenmacher-A.Voros $[\mathbf{N V}]$, Y. Colin de Verdière-B. Parisse $[\mathbf{C V P}]$, J. Toth and the author [TZ2] and outside of such tubes by N. Burq-M. Zworski $[\mathbf{B Z}]$ and H. Christianson [Chr]. Very striking and surprising studies of analogous behavior in the "quantum cat map" setting are in $[\mathbf{F N B}, \mathbf{F N}]$.

A few words on the limitations of this survey. Due to the author's lack of knowledge, the $L$-function or arithmetic methods in QUE will not be discussed in more than a cursory way. Rather we concentrate on the PDE or microlocal aspects of quantum ergodicity. By necessity, microlocal analytical proofs must make direct connections between spectrum and geodesic flow, and cannot by-pass this obstacle by taking a special arithmetic route. Moreover, we restrict the setting to Laplacians on Riemannian manifolds for simplicity of exposition, but it is just a special case of the semi-classical asymptotics (as the Planck constant tends to zero) of spectra of Schrödinger operators $h^{2} \Delta+V$. Schrödinger eigenfunctions are equally relevant to physicists and mathematics. Another important setting is that of quantum "cat maps" on tori and other Kähler manifolds. In this context, the quantum dynamics is defined by quantizations of symplectic maps of Kähler manifolds acting on spaces of holomorphic sections of powers of a positive line bundle. The mathematics is quite similar to the Riemannian setting, and results in the cat map setting often suggest analogues in the Riemannian setting. In particular, the scarring results of Faure-Nonnemacher- de Bièvre [FNB] and the variance results of Kurlberg-Rudnick [KR] and Schubert [Schu3] are very relevant to the scarring and variance results we present in the Riemannian setting.

Although we do not discuss it here, a good portion of the literature of quantum chaos is devoted to numerics and physicists' heuristics. Some classics are $[\mathbf{B e r}, \mathbf{H e}, \mathbf{H e 2}]$. The article of A. Barnett [Bar] (and in the 
cat map case $[\mathbf{F N B}, \mathbf{F N}]$ ) is a relatively recent discussion of the numerical results in quantum ergodicity. There is a wealth of phenomenology standing behind the rigorous results and heuristic proofs in this field. Much of it still lies far beyond the scope of the current mathematical techniques.

Finally, we thank N. Anantharaman, A. Hassell, and S. Nonnenmacher for explanations of important points in their work. We have incorporated many of their clarifications in the survey. We also thank K. Burns, H. Hezari, J. Franks, E. Lindenstrauss, P. Sarnak, J. A. Toth, A. Wilkinson and J. Wunsch for further clarifications and corrections. Of course, responsibility for any errors that remain is the author's.

\section{Wave equation and geodesic flow}

Classical mechanics is the study of Hamiltonians (real valued functions) on phase space (a symplectic manifold) and their Hamiltonian flows. Quantum mechanics is the study of Hermitian operators on Hilbert spaces and the unitary groups they generate. The model quantum Hamiltonians we will discuss are Laplacians $\Delta$ on compact Riemannian manifolds $(M, g)$ (with or without boundary). Throughout we denote the dimension of $M$ by $d=\operatorname{dim} M$.

1.1. Geodesic flow and classical mechanics. The classical phase space in this setting is the cotangent bundle $T^{*} M$ of $M$, equipped with its canonical symplectic form $\sum_{i} d x_{i} \wedge d \xi_{i}$. The metric defines the Hamiltonian $H(x, \xi)=|\xi|_{g}=\sqrt{\sum_{i j=1}^{n} g^{i j}(x) \xi_{i} \xi_{j}}$ on $T^{*} M$, where $g_{i j}=g\left(\frac{\partial}{\partial x_{i}}, \frac{\partial}{\partial x_{j}}\right),\left[g^{i j}\right]$ is the inverse matrix to $\left[g_{i j}\right]$. We denote the volume density of $(M, g)$ by $d V o l$ and the corresponding inner product on $L^{2}(M)$ by $\langle f, g\rangle$. The unit (co-) ball bundle is denoted $B^{*} M=\{(x, \xi):|\xi| \leq 1\}$.

The classical evolution (dynamics) is given by the geodesic flow of $(M, g)$, i.e. the Hamiltonian flow $g^{t}$ of $H$ on $T^{*} M$. Let $X_{H}$ denote the Hamiltonian vector field of $\mathrm{H}$. By definition, $g^{t}(x, \xi)=\left(x_{t}, \xi_{t}\right)$, where $\left(x_{t}, \xi_{t}\right)$ is the terminal tangent vector at time $t$ of the unit speed geodesic starting at $x$ in the direction $\xi$. Here and below, we often identify $T^{*} M$ with the tangent bundle $T M$ using the metric to simplify the geometric description. The geodesic flow preserves the energy surfaces $\{H=E\}$ which are the co-sphere bundles $S_{E}^{*} M$. Due to the homogeneity of $H$, the flow on any energy surface $\{H=E\}$ is equivalent to that on the co-sphere bundle $S^{*} M=\{H=1\}$.

We define the Liouville measure $\mu_{L}$ on $S^{*} M$ to be the surface measure $d \mu_{L}=\frac{d x d \xi}{d H}$ induced by the Hamiltonian $H=|\xi|_{g}$ and by the symplectic volume measure $d x d \xi$ on $T^{*} M$. The geodesic flow (like any Hamiltonian flow) preserves $d x d \xi$. It also preserves Liouville measue on $S^{*} M$.

1.2. Ergodic and Anosov geodesic flows. The geodesic flow is called ergodic if the unitary operator

$$
V^{t} a(\rho)=a\left(g^{t}(\rho)\right)
$$


on $L^{2}\left(S^{*} M, d \mu_{L}\right)$ has no invariant $L^{2}$ functions besides constant functions. Equivalently, any invariant set $E \subset S^{*} M$ has either zero measure or full measure.

A geodesic flow $g^{t}$ is called Anosov on on $S_{g}^{*} M$ if the tangent bundle $T S_{g}^{*} M$ splits into $g^{t}$ invariant sub-bundles $E^{u}(\rho) \oplus E^{s}(\rho) \oplus \mathbb{R} X_{H}(\rho)$ where $E^{u}$ is the unstable subspace and $E^{s}$ the stable subspace. They are defined by

$$
\begin{aligned}
& \left\|d g^{t} v\right\| \leq C e^{-\lambda t}\|v\|, \quad \forall v \in E^{s}, t \geq 0, \\
& \left\|d g^{t} v\right\| \leq C e^{\lambda t}\|v\|, \quad \forall v \in E^{u}, t \leq 0 .
\end{aligned}
$$

The sub-bundles are integrable and give stable, resp. unstable foliations $W^{s}, W^{u}$. The leaves through $x$ are denoted by $W^{s}(x), W^{u}(x)$. Thus, the geodesic flow contracts everything exponentially fast along the stable leaves and expands everything exponentially fast along the unstable leaves. Anosov geodesic flows are ergodic. We refer to $[\mathbf{K l}]$ for background.

The unstable Jacobian $J^{u}(\rho)$ at $\rho$ is defined by

$$
J^{u}(\rho)=\operatorname{det}\left(d g_{\mid E^{u}\left(g^{1} \rho\right)}^{-1}\right) .
$$

Define

$$
\begin{aligned}
\Lambda & =-\sup _{\rho \in S^{*} M} \log \left\|J^{u}(\rho)\right\| \\
& =\inf _{\mu \in \mathcal{M}_{I}}\left|\int_{S^{*} M} \log J^{u}(\rho) d \mu(\rho)\right|>0 .
\end{aligned}
$$

If $(M, g)$ is of dimension $d$ and constant curvature -1 then $\Lambda=d-1$. Here, $\mathcal{M}_{I}$ is the set of invariant probability measures for $g^{t}$.

The maximal expansion rate of the geodesic flow is defined by

$$
\lambda_{\max }=\lim _{t \rightarrow \infty} \frac{1}{t} \log \sup _{\rho \in S^{*} M}\left\|d g_{\rho}^{t}\right\| .
$$

The derivative $d g^{t}$ may be expressed in terms of Jacobi fields (see $[\mathbf{K l}]$, Lemma 3.1.17). In the hyperbolic (Anosov case), the stable/unstable subbundles are spanned by unstable Jacobi fields $Y_{1}^{u}, \ldots, Y_{d-1}^{u}$ whose norms grow exponentially as $t \rightarrow+\infty$ and decay exponentially as $t \rightarrow-\infty$, resp. stable Jacobi fields $Y_{1}^{s}, \ldots, Y_{d-1}^{s}$ whose norms behave in the opposite fashion. The unstable Jacobian is the norm $\left\|Y_{1}^{u} \wedge \cdots \wedge Y_{d-1}^{u}\right\|$.

1.3. Eigenfunctions and eigenvalues of $\Delta$. The quantization of the Hamiltonian $H$ is the square root $\sqrt{\Delta}$ of the positive Laplacian,

$$
\Delta=-\frac{1}{\sqrt{g}} \sum_{i, j=1}^{n} \frac{\partial}{\partial x_{i}} g^{i j} g \frac{\partial}{\partial x_{j}}
$$

of $(M, g)$. Here, $g=\operatorname{det}\left[g_{i j}\right]$. The eigenvalue problem on a compact Riemannian manifold

$$
\Delta \varphi_{j}=\lambda_{j}^{2} \varphi_{j}, \quad\left\langle\varphi_{j}, \varphi_{k}\right\rangle=\delta_{j k}
$$


is dual under the Fourier transform to the wave equation. Here, $\left\{\varphi_{j}\right\}$ is a choice of orthonormal basis of eigenfunctions, which is not unique if the eigenvalues have multiplicities $>1$. The individual eigenfunctions are difficult to study directly, and so one generally forms the spectral projections kernel,

$$
E(\lambda, x, y)=\sum_{j: \lambda_{j} \leq \lambda} \varphi_{j}(x) \varphi_{j}(y)
$$

Semi-classical asymptotics is the study of the $\lambda \rightarrow \infty$ limit of the spectral data $\left\{\varphi_{j}, \lambda_{j}\right\}$ or of $E(\lambda, x, y)$.

1.4. Eigenvalues and Planck's constant. Although it is only a notational issue in this setting, it is conceptually useful to identify inverse frequencies with Planck's constant, i.e. to define

$$
\hbar_{j}=\lambda_{j}^{-1} .
$$

The eigenvalue problem then takes the form of a Schrödinger equation

$$
\hbar_{j}^{2} \Delta \varphi_{j}=\varphi_{j}
$$

Thus the high frequency limit is a special case of the semi-classical limit $\hbar \rightarrow 0$.

1.5. Eigenvalue Multiplicities. The multiplicity of an eigenvalue $\lambda_{j}^{2}$ is the dimension

$$
m\left(\lambda_{j}\right)=\operatorname{dim} \operatorname{ker}\left(\Delta-\lambda_{j}^{2}\right)
$$

of the eigenspace. As will be discussed in $\S 2$, the general Weyl law gives the bound $m\left(\lambda_{j}\right) \leq C \lambda_{j}^{d-1}$ in dimension $d$. In the case of negatively curved manifolds, the bound has been improved by a $\left(\log \lambda_{j}\right)^{-1}$ factor. Thus it is possible for sequences of eigenvalues to have the huge multiplicities $\lambda_{j}^{d-1}\left(\log \lambda_{j}\right)^{-1}$. When such multiplicities occur, it is possible to build up superpositions of eigenfunctions which have special concentration properties (see §5). Hassell's scarring theorem is based on the non-existence of such multiplicities for certain sequences of eigenvalues $(\S 7)$.

In fact such multiplicities do occur in the quantum cap map setting for sparse sequences of eigenvalues and are responsible for the very strange behavior of eigenstates in that case $[\mathbf{F N B}, \mathbf{F N}]$. It is unknown if such sequences exist in the Riemannian Anosov setting. This is one of the fundamental obstacles to understanding whether or how scarring occurs in the Riemannian case. 
1.6. Quantum evolution. Quantum evolution is given by the wave group

$$
U^{t}=e^{i t \sqrt{\Delta}}
$$

which in a rigorous sense is the quantization of the geodesic flow $\Phi^{t}$. It is generated by the pseudo-differential operator $\sqrt{\Delta}$, as defined by the spectral theorem (it has the same eigenfunctions as $\Delta$ and the eigenvalues $\lambda_{j}$ ). The (Schwartz) kernel of the wave group on a compact Riemannian manifold can be represented in terms of the spectral data by

$$
U^{t}(x, y)=\sum_{j} e^{i t \lambda_{j}} \varphi_{j}(x) \varphi_{j}(y),
$$

or equivalently as the Fourier transform $\int_{\mathbb{R}} e^{i t \lambda} d E(\lambda, x, y)$ of the spectral projections. Hence spectral asymptotics is often studied through the large time behavior of the wave group.

Evolution can be studied on the level of "points" or on the level of observables. Evolution of points in classical mechanics gives the orbits or trajectories of the geodesic flow (i.e. the parameterized geodesics). Evolution $\psi_{t}=U^{t} \psi$ of wave functions gives the "Schrödinger picture" of quantum mechanics. Eigenfunctions arise in quantum mechanics as stationary states, i.e. states $\psi$ for which the probability measure $|\psi(t, x)|^{2} d v o l$ is constant where $\psi(t, x)=U^{t} \psi(x)$ is the evolving state. This follows from the fact that

$$
U^{t} \varphi_{k}=e^{i t \lambda_{k}} \varphi_{k}
$$

and that $\left|e^{i t \lambda_{k}}\right|=1$. By unitarity $U^{t *}=U^{-t}$.

As an alternative one could define the quantum evolution as in $[\mathbf{A N}]$ to be the semi-classical Schrödinger propagator

$$
U_{\hbar}^{t}:=e^{-i t \hbar \Delta}
$$

This replaces the homogeneous Fourier integral operator $U^{t}$ by a semiclassical Fourier integral operator. The theories are quite parallel; we mainly keep to the homogeneous theory in this survey (See $[\mathbf{D S j}]$ and $[\mathbf{E Z}]$ ) for background on these notions).

1.7. Observables. In the classical setting, observables are (real-valued) functions on $T^{*} M$ which we usually take to be homogeneous of degree zero (or as zeroth order symbols). In the quantum setting, observables are $\psi D O$ 's (pseudodifferential operators) of all orders; we often restrict to the subalgebra $\Psi^{0}$ of $\psi D O$ 's of order zero. We denote by $\Psi^{m}(M)$ the subspace of pseudodifferential operators of order $m$. The algebra is defined by constructing a quantization $O p$ from an algebra of symbols $a \in S^{m}\left(T^{*} M\right)$ of order $m$ (polyhomogeneous functions on $T^{*} M \backslash 0$ ) to $\Psi^{m}$. A function on $T^{*} M \backslash 0$ 
is called polyhomogeneous or a classical symbol of order $m, a \in S_{p h g}^{m}$, if it admits an asymptotic expansion,

$$
a(x, \xi) \sim \sum_{j=0}^{\infty} a_{m-j}(x, \xi), \quad \text { where } a_{k}(x, \tau \xi)=\tau^{k} a_{k}(x, \xi), \quad(|\xi| \geq 1)
$$

for some $m$ (called its order). The asympotics hold in the sense that

$$
a-\sum_{j=0}^{R} a_{m-j} \in S^{m-R-1},
$$

where $\sigma \in S^{k}$ if $\sup (1+|\xi|)^{j-k}\left|D_{x}^{\alpha} D_{\xi}^{\beta} \sigma(x, \xi)\right|<+\infty$ for all compact set and for all $\alpha, \beta, j$. There is a semi-classical analogue in the $\hbar$ setting where the complete symbol $a_{\hbar}(x, \xi)$ depends on $\hbar$ in a similar poly-homogeneous way,

$$
a_{\hbar} \sim \hbar^{-m} \sum_{j=0}^{R} \hbar^{j} a_{m-j} .
$$

We refer to [DSj, GSt, EZ, Dui] for background on microlocal analysis (or semi-classical analysis), i.e. for the theory of pseudo-differential and Fourier integral operators.

The main idea is that such observables have good classical limits. The high frequency limit is mirrored in the behavior of the symbol as $|\xi| \rightarrow \infty$.

An useful type of observable is a smooth cutoff $\chi_{U}(x, \xi)$ to some open set $U \subset S^{*} M$ (it is homogeneous so it cuts off to the cone through $U$ in $\left.T^{*} M\right)$. That is, a function which is one in a large ball in $U$ and zero outside $U$. Then $O p\left(\chi_{U}\right)$ is called a microlocal cutoff to $U$. Its expectation values in the states $\varphi_{k}$ give the phase space mass of the state in $U$ or equivalently the probability amplitude that the particle is in $U$. (The modulus square of the amplitude is the probability).

There are several standard notations for quantizations of symbols $a$ to operators $A$ that we use in this survey. We use the notation $O p(a)$ or $\hat{a}$ interchangeably.

1.8. Heisenberg uncertainty principle. The Heisenberg uncertainty principle is the heuristic principle that one cannot measure things in regions of phase space where the product of the widths in configuration and momentum directions is $\leq \hbar$.

It is useful to microlocalize to sets which shrink as $\hbar \rightarrow 0$ using $\hbar$ (or $\lambda$ )-dependent cutoffs such as $\chi_{U}\left(\lambda^{\delta}(x, \xi)\right)$. The Heisenberg uncertainty principle is manifested in pseudo-differential calculus in the difficulty (or impossibility) of defining pseudo-differential cut-off operators $O p\left(\chi_{U}\left(\lambda^{\delta}(x, \xi)\right)\right.$ when $\delta>\frac{1}{2}$. That is, such small-scale cutoffs do not obey the usual rules of semi-classical analysis (behavior of symbols under composition). The uncertainty principle allows one to study eigenfunctions by microlocal methods in 
configuration space balls $B\left(x_{0}, \lambda_{j}^{-1}\right)$ of radius $\hbar$ (since there is no constraint on the height) or in a phase space ball of radius $\lambda_{j}^{-1 / 2}$.

1.9. Matrix elements and Wigner distributions. These lectures are mainly concerned with the matrix elements $\left\langle O p(a) \varphi_{j}, \varphi_{k}\right\rangle$ of an observable relative to the eigenfunctions. The diagonal matrix elements

$$
\rho_{j}(O p(a)):=\left\langle O p(a) \varphi_{j}, \varphi_{j}\right\rangle
$$

are interpreted in quantum mechanics as the expected value of the observable $O p(a)$ in the energy state $\varphi_{j}$. The off-diagonal matrix elements are interpreted as transition amplitudes. Here, and below, an amplitude is a complex number whose modulus square is a probability.

If we fix the quantization $a \rightarrow O p(a)$, then the matrix elements can be represented by Wigner distributions. In the diagonal case, we define $W_{k} \in \mathcal{D}^{\prime}\left(S^{*} M\right)$ by

$$
\int_{S^{*} M} a d W_{k}:=\left\langle O p(a) \varphi_{k}, \varphi_{k}\right\rangle
$$

1.10. Evolution of Observables. Evolution of observables is particularly relevant in quantum chaos and is known in physics as the "Heisenberg picture".

The evolution of observables in the Heisenberg picture is defined by

$$
\alpha_{t}(A):=U^{t} A U^{-t}, \quad A \in \Psi^{m}(M) .
$$

and since Dirac's Principles of Quantum Mechanics, it was known to correspond to the classical evolution

$$
V^{t}(a):=a \circ g^{t}
$$

of observables $a \in C^{\infty}\left(S^{*} M\right)$. Egorov's theorem is the rigorous version of this correspondence: it says that $\alpha_{t}$ defines an order-preserving automorphism of $\Psi^{*}(M)$, i.e. $\alpha_{t}(A) \in \Psi^{m}(M)$ if $A \in \Psi^{m}(M)$, and that

$$
\sigma_{U_{t} A U_{t}^{*}}(x, \xi)=\sigma_{A}\left(g^{t}(x, \xi)\right):=V^{t}\left(\sigma_{A}\right), \quad(x, \xi) \in T^{*} M \backslash 0 .
$$

This formula is almost universally taken to be the definition of quantization of a flow or map in the physics literature.

1.11. Hadamard parametrix. The link between spectral theory and geometry, and the source of Egorov's theorem for the wave group, is the construction of a parametrix (or WKB formula) for the wave kernel. For small times $t$, the simplest is the Hadamard parametrix,

$$
U^{t}(x, y) \sim \int_{0}^{\infty} e^{i \theta\left(r^{2}(x, y)-t^{2}\right)} \sum_{k=0}^{\infty} U_{k}(x, y) \theta^{\frac{d-3}{2}-k} d \theta \quad(t<\operatorname{inj}(M, g))
$$


where $r(x, y)$ is the distance between points, $U_{0}(x, y)=\Theta^{-\frac{1}{2}}(x, y)$ is the volume 1/2-density, $\operatorname{inj}(M, g)$ is the injectivity radius, and the higher Hadamard coefficients are obtained by solving transport equations along geodesics. The parametrix is asymptotic to the wave kernel in the sense of smoothness, i.e. the difference of the two sides of (17) is smooth. The relation (17) may be iterated using $\left(U^{t}\right)^{m}=U^{t m}$ to obtain a parametrix for long times. For manifolds without conjugate points, the Hadamard parametrix is globally well defined on the universal cover and can then be summed over the deck transformation group to obtain a parametrix on the quotient.

Egorov's theorem (16) may be proved by explicitly writing out the integral representation for $U^{-t} A U^{t}$ using (17) and by applying the stationary phase method to simplify the integral.

1.12. Ehrenfest time. The aim in quantum chaos is to obtain information about the high energy asymptotics as $\lambda_{j} \rightarrow \infty$ of eigenvalues and eigenfunctions by connecting information about $U^{t}$ and $g^{t}$. The connection comes from comparing (9) and (17), or by using Egorov's theorem (16). But to use the hypothesis that $g^{t}$ is ergodic or chaotic, one needs to exploit the connection as $\hbar \rightarrow 0$ and $t \rightarrow \infty$. The difficulty in quantum chaos is that the approximation of $U^{t}$ by $g^{t}$ is only a good one for $t$ less than the Eherenfest time

$$
T_{E}=\frac{\log |\hbar|}{\lambda_{\max }},
$$

where $\lambda_{\max }$ is defined in (5).

Roughly speaking, the idea is that the evolution of a well constructed "coherent" quantum state or particle is a moving lump that "tracks along" the trajectory of a classical particle up to time $T_{H}$ and then slowly falls apart and stops acting like a classical particle. Numerical studies of long time dynamics of wave packets are given in works of E. J. Heller $[\mathbf{H e}, \mathbf{H e 2}]$ and rigorous treatments are in Bouzouina-Robert [BouR], Combescure-Robert [CR] and Schurbert [Schu3].

The basic result expressed in semi-classical notation is that there exists $\Gamma>0$ such that

$$
\left\|U_{\hbar}^{-t} O p(a) U_{\hbar}^{t}-O p\left(a \circ g^{t}\right)\right\| \leq C \hbar e^{t \Gamma}
$$

Such an estimate has long been known if $\Gamma$ is not specified in terms of the geodesics. It is implicit in $[\mathbf{B e}]$ and used explicitly in $[\mathbf{Z 4}]$, based on long time wave equation estimates of Volovoy. The best constant should be $\Gamma=\lambda_{\max }$.

Thus, one only expects good joint asymptotics as $\hbar \rightarrow 0, T \rightarrow \infty$ for $t \leq T_{E}$. As a result, one can only exploit the approximation of $U^{t}$ by $g^{t}$ for the relatively short time $T_{E}$. 
1.13. Modes, quasi-modes and coherent states. This survey is mainly concerned with eigenfunctions. But one of the main tools for studying eigenfunctions is the construction of quasi-modes or approximate eigenfunctions. Lagrangian and coherent states are closely related to quasi-modes. We refer to [Dui, CV2, B.B, Ra, Ra2] for background.

A quasi-mode $\left\{\psi_{k}\right\}$ of order zero is a sequence of $L^{2}$-normalized functions satisfying

$$
\left\|\left(\Delta-\mu_{k}\right) \psi_{k}\right\|_{L^{2}}=O(1)
$$

for a sequence of quasi-eigenvalues $\mu_{k}$. By the spectral theorem, there must exist true eigenvalues in the interval $\left[\mu_{k}-\delta, \mu_{k}+\delta\right]$ for some $\delta>0$. Moreover, if $E_{k, \delta}$ denotes the spectral projection for the Laplacian corresponding to this interval, then

$$
\left\|E_{k, \delta} \psi_{k}-\psi_{k}\right\|_{L^{2}}=O\left(k^{-1}\right) .
$$

A more general definition is that a quasi-mode of order $s$ is a sequence satisfying

$$
\left\|\left(\Delta-\mu_{k}\right) \psi_{k}\right\|_{L^{2}}=O\left(\mu_{k}^{-s}\right)
$$

Then

$$
\left\|E_{k, \delta} \psi_{k}-\psi_{k}\right\|_{L^{2}}=O\left(k^{-s-1}\right) .
$$

This definition allows for very weak versions of quasi-modes, e.g. quasimodes of order -1 . They carry no information about eigenvalues since there always exist eigenvalues in intervals of width one. However, they do carry some information on eigenfunctions.

In references such as [Dui], quasi-modes are constructed in the form of oscillatory integrals or Lagrangian states,

$$
\psi_{\lambda}(x)=\int_{\mathbb{R}^{k}} e^{i \lambda S(x, \xi)} a(x, \xi) d \xi .
$$

The phase generates the Lagrangian submanifold

$$
\Lambda_{S}=\left\{\left(x, d_{x} S\right): d_{\xi} S(x, \xi)=0\right\} \subset T^{*} M .
$$

If $\Lambda \subset S^{*} M$ is a closed Lagrangian submanifold invariant under the geodesic flow then $\psi_{\lambda}$ is a quasi-mode of order -1 . In favorable circumstances, one can further determine the amplitude so that $\left\|\left(-\Delta-\lambda^{2}\right) \psi_{\lambda}\right\|=O\left(\lambda^{-s}\right)$ and construct a high order quasi-mode. Such a quasi-mode is the "quantization" of $\Lambda$ and the associated sequence concentrates on $\Lambda$.

A coherent state is a special kind of Lagrangian state, or more precisely isotropic state, which quantizes a "point" in phase space rather than a Lagrangian submanifold. The simplest examples are the states $\varphi_{\hbar}^{x_{0}, \xi_{0}}(x)=$ $\hbar^{-n / 2} e^{\frac{i}{\hbar}\left\langle x, \xi_{0}\right\rangle} e^{-\frac{\left|x-x_{0}\right|^{2}}{\hbar}}$ on $\mathbb{R}^{n}$, which are localized to the smallest possible volume in phase space, namely a unit cube of volume $\hbar$ around $\left(x_{0}, \xi_{0}\right)$. They are Lagrangian states with complex phases associated to positive Lagrangian submanifolds of complexified phase space. 
1.14. Riemannian random waves. Eigenfunctions in the chaotic case are never similar to such quasi-modes or Lagrangian states. Rather, they are conjectured to resemble random waves. This heuristic was first proposed by M. V. Berry [Ber], who had in mind random Euclidean plane waves. The specific model discussed here was used by the author to show that eigenfunctions in the ergodic case behave to some degree like random orthonormal bases of spherical harmonics. We use the term Riemannian random waves for this model.

We decompose the spectrum of a compact Riemannian manifold $(M, g)$ into intervals $I_{N}=[N, N+1]$ and define the finite dimensional Hilbert spaces $\mathcal{H}_{I_{N}}$ as the space of linear combinations of eigenfunctions with frequencies in an interval $I_{N}:=[N, N+1]$. The precise decomposition of $\mathbb{R}$ into intervals is not canonical and any choice of intervals of finite width would work as long as the set of closed geodesics of $(M, g)$ has measure zero. We will not discuss the other case since it has no bearing on quantum ergodicity. We denote by $\left\{\varphi_{N j}\right\}_{j=1}^{d_{N}}$ an orthonormal basis of $\mathcal{H}_{I_{N}}$ where $d_{N}=\operatorname{dim} \mathcal{H}_{I_{N}}$. We endow the real vector space $\mathcal{H}_{I_{N}}$ with the Gaussian probability measure $\gamma_{N}$ defined by

$$
\gamma_{N}(s)=\left(\frac{d_{N}}{\pi}\right)^{d_{N} / 2} e^{-d_{N}|c|^{2}} d c, \quad \psi=\sum_{j=1}^{d_{N}} c_{j} \varphi_{N j}, \quad d_{N}=\operatorname{dim} \mathcal{H}_{I_{N}}
$$

Here, $d c$ is $d_{N}$-dimensional real Lebesgue measure. The normalization is chosen so that $\mathbb{E}_{\gamma_{N}}\langle\psi, \psi\rangle=1$, where $\mathbb{E}_{\gamma_{N}}$ is the expected value with respect to $\gamma_{N}$. Equivalently, the $d_{N}$ real variables $c_{j}\left(j=1, \ldots, d_{N}\right)$ are independent identically distributed (i.i.d.) random variables with mean 0 and variance $\frac{1}{2 d_{N}}$; i.e.

$$
\mathbb{E}_{\gamma_{N}} c_{j}=0, \quad \mathbb{E}_{\gamma_{N}} c_{j} c_{k}=\frac{1}{d_{N}} \delta_{j k}
$$

We note that the Gaussian ensemble is equivalent to picking $\psi_{N} \in \mathcal{H}_{I_{N}}$ at random from the unit sphere in $\mathcal{H}_{I_{N}}$ with respect to the $L^{2}$ inner product.

Numerical results confirming some features of the random wave model are given in $[\mathbf{H R}]$.

1.15. Hyperbolic plane. It is useful to have one concrete example in mind, and we will use the hyperbolic plane $\mathcal{H}$ or disc $\mathbf{D}$ as a running model. In the disc model $\mathbf{D}=\{z \in \mathbb{C},|z|<1\}$, the hyperbolic metric has the form $d s^{2}=\frac{4|d z|^{2}}{\left(1-|z|^{2}\right)^{2}}$. Its isometry group is $G=P S U(1,1)$; the stabilizer of 0 is $K \simeq S O(2)$ and thus $\mathbf{D} \simeq G / K$. Without comment we will also identify D with the upper half plane and $G$ with $P S L(2, \mathbb{R})$. In hyperbolic polar coordinates centered at the origin 0 , the (positive) Laplacian is the operator

$$
-\triangle=\frac{\partial^{2}}{\partial r^{2}}+\operatorname{coth} r \frac{\partial}{\partial r}+\frac{1}{\sinh ^{2} r} \frac{\partial^{2}}{\partial \theta^{2}} .
$$


The distance on $\mathbf{D}$ induced by the Riemannian metric will be denoted $d_{\mathbf{D}}$. We denote the volume form by $d(z)$.

The unit tangent bundle $S \mathbf{D}$ of the hyperbolic disc $\mathbf{D}$ may be identifed with the unit cosphere bundle $S^{*} \mathbf{D}$ by means of the metric. We further identify $S \mathbf{D} \equiv P S U(1,1)$, since $P S U(1,1)$ acts freely and transitively on $S$ D. We identify a unit tangent vector $(z, v)$ with a group element $g$ if $g \cdot(0,(1,0))=(z, v)$.

We denote by $B=\{z \in \mathbb{C},|z|=1\}$ the boundary at infinity of $\mathbf{D}$. Then we can also identify $S \mathbf{D} \equiv \mathbf{D} \times B$. Here, we identify $(z, b) \in \mathbf{D} \times B$ with the unit tangent vector $(z, v)$, where $v \in S_{z} \mathbf{D}$ is the vector tangent to the unique geodesic through $z$ ending at $b$.

The geodesic flow $g^{t}$ on $S \mathbf{D}$ is defined by $g^{t}(z, v)=\left(\gamma_{v}(t), \gamma_{v}^{\prime}(t)\right)$ where $\gamma_{v}(t)$ is the unit speed geodesic with initial value $(z, v)$. The space of geodesics is the quotient of $S \mathbf{D}$ by the action of $g^{t}$. Each geodesic has a forward endpoint $b$ and a backward endpoint $b^{\prime}$ in $B$, hence the space of geodesics of $\mathbf{D}$ may be identified with $B \times B \backslash \Delta$, where $\Delta$ denotes the diagonal in $B \times B$ : To $\left(b^{\prime}, b\right) \in B \times B \backslash \Delta$ there corresponds a unique geodesic $\gamma_{b^{\prime}, b}$ whose forward endpoint at infinity equals $b$ and whose backward endpoint equals $b^{\prime}$. We then have the identification

$$
S \mathbf{D} \equiv(B \times B \backslash \Delta) \times \mathbb{R}
$$

The choice of time parameter is defined - for instance - as follows: The point $\left(b^{\prime}, b, 0\right)$ is by definition the closest point to 0 on $\gamma_{b^{\prime}, b}$ and $\left(b^{\prime}, b, t\right)$ denotes the point $t$ units from $\left(b, b^{\prime}, 0\right)$ in signed distance towards $b$.

1.15.1. Dynamics and group theory of $G=S L(2, \mathbb{R})$. The generators of $s l(2, \mathbb{R})$ are denoted by

$$
H=\left(\begin{array}{cc}
1 & 0 \\
0 & -1
\end{array}\right), \quad V=\left(\begin{array}{ll}
0 & 1 \\
1 & 0
\end{array}\right), \quad W=\left(\begin{array}{cc}
0 & -1 \\
1 & 0
\end{array}\right) .
$$

We denote the associated one parameter subgroups by $A, A_{-}, K$. We denote the raising/lowering operators for $K$-weights by

$$
E^{+}=H+i V, \quad E^{-}=H-i V .
$$

The Casimir operator is then given by $4 \Omega=H^{2}+V^{2}-W^{2}$; on $K$-invariant functions, the Casimir operator acts as the laplacian $\triangle$. We also put

$$
X_{+}=\left(\begin{array}{ll}
0 & 1 \\
0 & 0
\end{array}\right), \quad X_{-}=\left(\begin{array}{ll}
0 & 0 \\
1 & 0
\end{array}\right),
$$

and denote the associated subgroups by $N, N_{-}$. In the identification $S \mathbf{D} \equiv$ $P S L(2, \mathbb{R})$ the geodesic flow is given by the right action $g^{t}(g)=g a_{t}$ of $A$, resp. the horocycle flow $\left(h^{u}\right)_{u \in \mathbb{R}}$ is defined by $h^{u}(g)=g n_{u}$, where

$$
a_{t}=\left(\begin{array}{cc}
e^{t / 2} & 0 \\
0 & e^{-t / 2}
\end{array}\right), \quad n_{u}=\left(\begin{array}{cc}
1 & u \\
0 & 1
\end{array}\right) .
$$


The closed orbits of the geodesic flow $g^{t}$ on $\Gamma \backslash G$ are denoted $\{\gamma\}$ and are in one-to-one correspondence with the conjugacy classes of hyperbolic elements of $\Gamma$. We denote by $G_{\gamma}$, respectively $\Gamma_{\gamma}$, the centralizer of $\gamma$ in $G$, respectively $\Gamma$. The group $\Gamma_{\gamma}$ is generated by an element $\gamma_{0}$ which is called a primitive hyperbolic geodesic. The length of $\gamma$ is denoted $L_{\gamma}>0$ and means that $\gamma$ is conjugate, in $G$, to

$$
a_{\gamma}=\left(\begin{array}{cc}
e^{L_{\gamma} / 2} & 0 \\
0 & e^{-L_{\gamma} / 2}
\end{array}\right) .
$$

If $\gamma=\gamma_{0}^{k}$ where $\gamma_{0}$ is primitive, then we call $L_{\gamma_{0}}$ the primitive length of the closed geodesic $\gamma$.

1.15.2. Non-Euclidean Fourier analysis. Following [Hel], we denote by $\langle z, b\rangle$ the signed distance to 0 of the horocycle through the points $z \in \mathbf{D}, b \in$ $B$. Equivalently,

$$
e^{\langle z, b\rangle}=\frac{1-|z|^{2}}{|z-b|^{2}}=P_{\mathbf{D}}(z, b),
$$

where $P_{\mathbf{D}}(z, b)$ is the Poisson kernel of the unit disc. We denote Lebesgue measure on $B$ by $|d b|$. We then introduce the hyperbolic plane waves $e^{\left(\frac{1}{2}+i r\right)\langle z, b\rangle}$, the analogues of the Euclidean plane waves $e^{i\langle x, \xi\rangle}$.

The non-Euclidean Fourier transform $\mathcal{F}: C_{c} \mathbf{D} \rightarrow C\left(B \times \mathbb{R}_{+}\right)$is defined by

$$
\mathcal{F} u(r, b)=\int_{\mathbf{D}} e^{\left(\frac{1}{2}-i r\right)\langle z, b\rangle} u(z) d \operatorname{Vol}(z) .
$$

The inverse Fourier transform is given by

$$
\mathcal{F}^{-1} g(z)=\int_{B} \int_{\mathbb{R}} e^{\left(\frac{1}{2}+i r\right)\langle z, b\rangle} g(r, b) r \tanh (2 \pi r) d r|d b| .
$$

The integration measure is the Plancherel measure. $\mathcal{F}$ extends to an isometry $\mathcal{F}: L^{2}(\mathbf{D}, d V) \rightarrow L^{2}\left(B \times \mathbb{R}_{+}, r \tanh (2 \pi r) d r d b\right)$.

1.15.3. Wave kernel on $\mathbf{D}$ and wave kernel on $\mathbf{X}_{\Gamma}$. The wave group $U^{t}=e^{i t \sqrt{\Delta}}$ of the positive Laplacian on $\mathbf{D}$ is thus given in terms of Fourier analysis by (distribution) integral

$$
U^{t}(z, w)=\int_{B} \int_{\mathbb{R}} e^{i\left(\frac{1}{4}+r^{2}\right) t} e^{\left(\frac{1}{2}+i r\right)\langle z, b\rangle} e^{\left(-\frac{1}{2}+i r\right)\langle w, b\rangle} r \tanh (2 \pi r) d r|d b| .
$$

The integral over $B$ may be eliminated by the stationary phase method to produce an integral over $\mathbb{R}$ alone; it is the Hadamard parametrix (17) in this case.

Now let $\Gamma \subset G$ be a discrete subgroup. In applications to quantum chaos we assume the quotient $\mathbf{X}_{\Gamma}$ is compact or of finite area. 
The wave kernel on the quotient $\mathbf{X}_{\Gamma}$ is obtained by automorphizing the wave kernel on $\mathbf{D}$ :

$$
U_{\Gamma}^{t}(z, w)=\sum_{\gamma \in \Gamma} U_{\Gamma}^{t}(z, \gamma w) .
$$

This representation is one of the inputs into the Selberg trace formula. The elements of $\Gamma$ are grouped into conjugacy classes $[\gamma] \in[\Gamma]$. The conjugacy classes correspond to closed geodesics of $\mathbf{X}_{\Gamma}$, i.e. periodic orbits of $g^{t}$ on $S^{*} \mathbf{X}_{\Gamma}$.

Let

$$
\Theta_{\Gamma}(T)=\#\left\{[\gamma]: L_{\gamma} \leq T\right\} .
$$

The prime geodesic theorem asserts that

$$
\Theta_{\Gamma}(T) \sim \frac{e^{h_{t o p} T}}{T},
$$

where $h_{t o p}$ is the topological entropy of $g^{t}$. In fact, $h_{t o p}=1$ for the hyperbolic case. The exponential growth of the length spectrum reflects the exponential growth of the geodesic flow.

The Ehrenfest time (18) is implicit in the exponential growth rate of $\Theta_{\Gamma}(T)$.

1.15.4. Representation theory of $G$ and spectral theory of $\triangle$. Let $\Gamma \subset G$ be a co-compact discrete subgroup, and let us consider the automorphic eigenvalue problem on $G / K$ :

$$
\left\{\begin{array}{l}
\triangle \varphi=\left(\frac{1}{4}+r^{2}\right) \varphi \\
\varphi(\gamma z)=\varphi(z) \text { for all } \gamma \in \Gamma \text { and for all } z
\end{array}\right.
$$

The solutions are the eigenfunctions of the Laplacian on the compact surface $\mathbf{X}_{\Gamma}=\Gamma \backslash G / K$. A standard notation for the eigenvalues is $\lambda^{2}=s(1-s)$ where $s=\frac{1}{2}+i r$.

Eigenfunctions of the Laplacian are closely connected with the representation theory of $G$ on $G / \Gamma$. We briefly describe the representation theory since the problems in quantum chaos have analogues in the discrete series as well as the unitary prinicipal series.

In the compact case, we have the decomposition into irreducibles,

$$
L^{2}(\Gamma \backslash G)=\bigoplus_{j=1}^{S} \mathcal{C}_{i r_{j}} \oplus \bigoplus_{j=0}^{\infty} \mathcal{P}_{i r_{j}} \oplus \bigoplus_{m=2, m \text { even }}^{\infty} \mu_{\Gamma}(m) \mathcal{D}_{m}^{+} \oplus \bigoplus_{m=2, m \text { even }}^{\infty} \mu_{\Gamma}(m) \mathcal{D}_{m}^{-},
$$

where $\mathcal{C}_{i r_{j}}$ denotes the complementary series representation, respectively $\mathcal{P}_{i r_{j}}$ denotes the unitary principal series representation, in which the Casimir operator $-\Omega=-\left(H^{2}+V^{2}-W^{2}\right)$ equals $s_{j}\left(1-s_{j}\right)=\frac{1}{4}+r_{j}^{2}$. In the complementary series case, $i r_{j} \in \mathbb{R}$ while in the principal series case $i r_{j} \in$ $i \mathbb{R}^{+}$. The irreducibles are indexed by their $K$-invariant vectors $\left\{\varphi_{i r_{j}}\right\}$, which 
is assumed to be the given orthonormal basis of $\triangle$-eigenfunctions. Thus, the multiplicity of $\mathcal{P}_{i r_{j}}$ is the same as the multiplicity of the corresponding eigenvalue of $\triangle$.

Further, $\mathcal{D}_{m}^{ \pm}$denotes the holomorphic (respectively anti-holomorphic) discrete series representation with lowest (respectively highest) weight $m$, and $\mu_{\Gamma}(m)$ denotes its multiplicity; it depends only on the genus of $\mathbf{X}_{\Gamma}$. We denote by $\psi_{m, j}\left(j=1, \ldots, \mu_{\Gamma}(m)\right)$ a choice of orthonormal basis of the lowest weight vectors of $\mu_{\Gamma}(m) \mathcal{D}_{m}^{+}$and write $\mu_{\Gamma}(m) \mathcal{D}_{m}^{+}=\oplus_{j=1}^{\mu_{\Gamma}(m)} \mathcal{D}_{m, j}^{+}$ accordingly.

There is a a direct integral decomposition for co-finite subgroups such as $\Gamma=P S L(2, \mathbb{Z})$ or congruence subgroups. The non-compactness gives rise to a continuous spectral subspace of Eisenstein series and a discrete spectral subspace of cuspidal eigenfunctions (which is only known to be non-trivial in the case of arithmetic $\Gamma$ ).

1.15.5. Helgason Poisson formula. The Fourier transform of an $L^{2}$ function on $\mathbf{D}$ is an $L^{2}$ function on $B \times \mathbb{R}_{+}$. There is an extension of the Fourier transform and the inversion formula to tempered distributions. We only consider the case of $\Gamma$-automorphic eigenfunctions where $\Gamma$ is co-compact. One then has $\mathcal{F} \varphi_{i r_{j}}=d T_{i r_{j}}(b) \otimes \delta_{r_{j}}(r)$, where $d T_{i r_{j}}$ is a distribution on the ideal boundary $B$. The inversion formula is Helgason's Poisson formula,

$$
\varphi_{i r}(z)=\int_{B} e^{\left(\frac{1}{2}+i r\right)\langle z, b\rangle} T_{i r, \varphi_{i r}}(d b),
$$

for all $z \in \mathbf{D}$. The kernel $e^{\left(\frac{1}{2}+i r\right)\langle z, b\rangle}=P_{\mathbf{D}}^{\left(\frac{1}{2}+i r\right)}(z, b)$ is called the generalized Poisson kernel. The distribution

$$
T_{i r, \varphi_{i r}}(d b)=\sum_{n \in \mathbb{Z}} a_{n}(r) b^{n}|d b| .
$$

is called the boundary value of $\varphi_{i r}$ and is obtained from the Fourier expansion

$$
\varphi_{i r}(z)=\sum_{n \in \mathbb{Z}} a_{n}(r) \Phi_{r, n}(z),
$$

of $\varphi_{i r}$ in the disc model in terms of the generalized spherical functions,

$$
e^{\left(\frac{1}{2}+i r\right)\langle z, b\rangle}=\sum_{n \in \mathbb{Z}} \Phi_{r, n}(z) b^{n}, \quad b \in B .
$$

Equivalently, the $\Phi_{r, n}$ are the joint eigenfunctions of $\Delta$ and of $K$.

When $\varphi_{i r_{j}}$ is a $\Gamma$-invariant eigenfunction, the boundary values $T_{i r_{j}}(d b)$ have the following invariance property:

$$
\begin{aligned}
\varphi_{i r_{j}}(\gamma z)=\varphi_{i r_{j}}(z) & \Longrightarrow e^{\left(\frac{1}{2}+i r_{j}\right)\langle\gamma z, \gamma b\rangle} T_{i r_{j}}(d \gamma b)=e^{\left(\frac{1}{2}+i r_{j}\right)\langle z, b\rangle} T_{i r_{j}}(d b) \\
& \Longrightarrow T_{i r_{j}}(d \gamma b)=e^{-\left(\frac{1}{2}+i r_{j}\right)\langle\gamma \cdot 0, \gamma \cdot b\rangle} T_{i r_{j}}(d b)
\end{aligned}
$$


This follows by the identities

$$
\langle g \cdot z, g \cdot b\rangle=\langle z, b\rangle+\langle g \cdot 0, g \cdot b\rangle,
$$

which implies

$$
P_{\mathbf{D}}(g z, g b)|d(g b)|=P_{\mathbf{D}}(z, b)|d b| .
$$

An interesting heuristic related to Berry's random wave hypothesis (see $\S 1.14)$ is the conjecture that the Fourier coefficients $a_{n}(r)$ in (30) should behave like independent Gaussian random variables of mean zero and variance one. This conjecture was stated explicitly and tested numerically by Aurich-Steiner in $[\mathbf{A S}]$. Related tests of the random wave model for hyperbolic quotients are in Hejhal-Rackner [HR].

Otal $[\mathbf{O}]$ and Schmid $[\mathbf{S c h}]$ have shown that $T_{i r_{j}}(d b)$ is the derivative of a Hölder $C^{\frac{1}{2}}$ continuous function $F_{i r_{j}}$ on $B$. Since its zeroth Fourier coefficient is non-zero, $T_{i r_{j}}(d b)$ is not literally the derivative of a periodic function, but it is the derivative of a function $F_{i r_{j}}$ on $\mathbb{R}$ satisfying $F_{i r_{j}}(\theta+2 \pi)=F_{i r_{j}}(\theta)+C_{j}$ for all $\theta \in \mathbb{R}$. Recall that for $0 \leq \delta \leq 1$ we say that a $2 \pi$-periodic function $F: \mathbb{R} \rightarrow \mathbb{C}$ is $\delta$-Hölder if $\left|F(\theta)-F\left(\theta^{\prime}\right)\right| \leq C\left|\theta-\theta^{\prime}\right|^{\delta}$. The smallest constant is denoted $\|F\|_{\delta}$ and $\Lambda_{\delta}$ denotes the Banach space of $\delta$-Hölder functions, up to additive constants.

1.15.6. Boundary values and representation theory $[\mathbf{Z 3}]$. The distributions $d T_{i r_{j}}$ have a natural interpretation in representation theory. We define $e_{i r_{j}} \in \mathcal{D}^{\prime}(\Gamma \backslash P S L(2, \mathbb{R}))$ such that

$$
e^{\left(\frac{1}{2}+i r_{j}\right)\langle z, b\rangle} T_{i r_{j}}(d b)=e_{i r_{j}}(z, b) P(z, b) d b .
$$

The distribution $e_{i r_{j}}$ is horocyclic-invariant and $\Gamma$-invariant. It may be expanded in a $K$-Fourier series,

$$
e_{i r_{j}}=\sum_{n \in \mathbb{Z}} \varphi_{i r_{j}, n}
$$

and it is easily seen (cf. [Z2]) that $\varphi_{i r_{j}, 0}=\varphi_{i r_{j}}$ and that $\varphi_{i r_{j}, n}$ is obtained by applying the $n$th normalized raising or lowering operator (Maass operator) $E^{ \pm}=H \pm i V$ to $\varphi_{i r_{j}}$. More precisely, one applies $\left(E^{ \pm}\right)^{n}$ and multiplies by the normalizing factor $\beta_{2 i r_{j}, n}=\frac{1}{\left(2 i r_{j}+1 \pm 2 n\right) \cdots\left(2 i r_{j}+1 \pm 2\right)}$.

\section{Weyl law and local Weyl law}

We now return to the general case. A basic result in semi-classical asymptotics is Weyl's law on counting eigenvalues. Intuitively, Weyl's law says that the trace of the spectral projector (6) has the asymptotics,

$$
\operatorname{Tr} E_{\lambda} \sim \frac{\operatorname{Vol}\left(|\xi|_{g} \leq \lambda\right)}{(2 \pi)^{d}},
$$

where $d=\operatorname{dim} M$, where $V o l$ is the symplectic volume measure relative to the natural symplectic form $\sum_{j=1}^{d} d x_{j} \wedge d \xi_{j}$ on $T^{*} M$. Thus, the dimension 
of the space where $H=\sqrt{\Delta}$ is $\leq \lambda$ is asymptotically the volume where its symbol $|\xi|_{g} \leq \lambda$. More precisely, on a manifold $M$ (possibly with boundary),

$$
\begin{aligned}
N(\lambda)= & \#\left\{j: \lambda_{j} \leq \lambda\right\} \\
= & \frac{\left|B_{d}\right|}{(2 \pi)^{d}} \operatorname{Vol}(M, g) \lambda^{d}+\frac{\left|B_{d-1}\right|}{(2 \pi)^{d-1}} \operatorname{Vol}(\partial M, g) \lambda^{d-1}+R(\lambda), \\
& \quad \text { where } \quad R(\lambda)=O\left(\lambda^{d-1}\right) .
\end{aligned}
$$

Here, $\left|B_{d}\right|$ is the Euclidean volume of the unit ball and $\operatorname{Vol}(M, g)$ (resp. $\operatorname{Vol}(\partial M, g)$ is the volume of $M$ (resp. $\partial M)$ with respect to the metric $g$. The growth of the remainder term depends on the long time behavior of $g^{t}$. It is of order $\lambda^{d-1}$ on the standard sphere or hemisphere, where all geodesics are periodic. By a classical theorem due to Duistermaat-Guillemin [D.G] (in the boundaryless case) and to Ivrii (in the boundary case),

$$
\begin{aligned}
& R(\lambda)=o\left(\lambda^{d-1}\right), \text { when the set of periodic geodesics } \\
& \text { has Liouville measure zero. }
\end{aligned}
$$

The remainder is then of smaller order than the derivative of the principal term, and in the boundary case, of smaller order than the boundary contribution. In this generic case, one has asymptotics in shorter intervals: (39)

$$
N([\lambda, \lambda+1])=\#\left\{j: \lambda_{j} \in[\lambda, \lambda+1]\right\}=n \frac{\left|B_{d}\right|}{(2 \pi)^{d}} \operatorname{Vol}(M, g) \lambda^{d-1}+o\left(\lambda^{d-1}\right) .
$$

Then the mean spacing between the eigenvalues in this interval is $\sim C_{d} \operatorname{Vol}(M, g)^{-1} \lambda^{-(d-1)}$, where $C_{d}$ is a constant depending on the dimension.

In the case of compact Riemannian manifolds of negative curvature without boundary, a sharper estimate of the remainder is possible:

$$
R(\lambda)=O\left(\frac{\lambda^{d-1}}{\log \lambda}\right) .
$$

This remainder is proved using (25) and the exponential growth rate in (27). The estimate (40) was proved by Selberg in the case of compact hyperbolic quotients and was generalized to all compact Riemannian manifolds without conjugate points by Bérard [Be]. The logarithm in the remainder is a direct outcome of the fact that one can only use the geodesic approximation to $U^{t}$ up to the Ehrenfest time $T_{E}$ (18).

This estimate has not been improved in fifty years, and there are no better results in the constant curvature case than in the general negatively curved case. The remainder estimate does not rule out the implausibleseeming scenario that in the interval $[\lambda, \lambda+1]$ there are only $\log \lambda$ distinct eigenvalues with multiplicities $\frac{\lambda^{d-1}}{\log \lambda}$. In fact, such implausibly large multiplicities do occur for a sparse set of "Planck constants" in the analogous case of quantizations of hyperbolic toral automorphisms (see $[\mathbf{F N B}]$ ). The 
issue of possibly high multiplicity or clustering of eigenvalues is important in Hassell's scarring result as well as the Anantharaman-Nonnenmacher results on entropies.

An important generalization is the local Weyl law concerning the traces $\operatorname{Tr} A E(\lambda)$ where $A \in \Psi^{m}(M)$. When $\partial M=\emptyset$ (which we henceforth assume unless explicitly stating otherwise), it asserts that

$$
\sum_{\lambda_{j} \leq \lambda}\left\langle A \varphi_{j}, \varphi_{j}\right\rangle=\frac{1}{(2 \pi)^{d}}\left(\int_{B^{*} M} \sigma_{A} d x d \xi\right) \lambda^{d}+O\left(\lambda^{d-1}\right) .
$$

There is also a pointwise local Weyl law:

$$
\sum_{\lambda_{j} \leq \lambda}\left|\varphi_{j}(x)\right|^{2}=\frac{1}{(2 \pi)^{d}}\left|B^{d}\right| \lambda^{d}+R(\lambda, x)
$$

where $R(\lambda, x)=O\left(\lambda^{d-1}\right)$ uniformly in $x$. When the periodic geodesics form a set of measure zero in $S^{*} M$, the remainders are of order $o\left(\lambda^{d-1}\right)$, and one could average over the shorter interval $[\lambda, \lambda+1]$. In the negatively curved case,

$$
R(\lambda, x)=O\left(\frac{\lambda^{d-1}}{\log \lambda}\right)
$$

Combining the Weyl and local Weyl law, we find the surface average of $\sigma_{A}$ is a limit of traces:

$$
\begin{aligned}
\omega(A) & :=\frac{1}{\mu_{L}\left(S^{*} M\right)} \int_{S^{*} M} \sigma_{A} d \mu_{L} \\
& =\lim _{\lambda \rightarrow \infty} \frac{1}{N(\lambda)} \sum_{\lambda_{j} \leq \lambda}\left\langle A \varphi_{j}, \varphi_{j}\right\rangle
\end{aligned}
$$

Here, $d \mu_{L}$ is Liouville measure on $S^{*} M(\S 1.1)$.

\section{Invariant states defined by eigenfunctions and their quantum limits}

When speaking of "states" in quantum mechanics, one might refer to a normalized wave function $\psi$ or alternatively to the matrix elements $\rho_{\psi}(A)=$ $\langle A \psi, \psi\rangle$ of an observable in the state. The latter use of "state" is a standard notion in $\mathrm{C}^{*}$ algebras, and is central in quantum ergodicity and mixing. The states evidently have the properties: (i) $\rho_{\psi}\left(A^{*} A\right) \geq 0 ;(i i) \rho_{\psi}(I)=1 ;(i i i) \rho_{\psi}$ is continuous in the norm topology. The classical analogue is a probability measure, viewed as a positive normalized linear functional on $C\left(S^{*} M\right)$. In particular, eigenfunctions define states on $\Psi^{0}$ (more correctly, its closure in the norm topology) as in (12), which we repeat:

$$
\rho_{k}(A)=\left\langle A \varphi_{k}, \varphi_{k}\right\rangle \text {. }
$$


We could (and will) also consider "transition amplitudes" $\rho_{j, k}(A)$ $=\left\langle A \varphi_{j}, \varphi_{k}\right\rangle$.

It is an immediate consequence of the fact that $U^{t} \varphi_{j}=e^{i t \lambda_{j}} \varphi_{j}$ that the diagonal states $\rho_{k}$ are invariant under the automorphism $\alpha_{t}(14)$ :

$$
\rho_{k}\left(U_{t} A U_{t}^{*}\right)=\rho_{k}(A) .
$$

In general, we denote by $\mathcal{E}$ the compact, convex set of states in the vector space of continuous linear functionals on the closure of $\Psi^{0}$ in its norm topology. We denote by

$$
\mathcal{E}_{\mathbb{R}}=\left\{\rho \in \mathcal{E}: \rho \circ \alpha_{t}=\rho\right\}
$$

the compact convex set of invariant states. For simplicity of notation, we continue to denote the closure by $\Psi^{0}$, and refer to $[\mathbf{Z 6}]$ for a detailed exposition.

From a mathematical point of view, such the states $\rho_{k}(A)$ are important because they provide the simplest means of studying eigenfunctions: one tests eigenfunctions against observables by studying the values $\rho_{k}(A)$. Many standard inequalities in PDE (e.g. Carleman estimates) have the form of testing eigenfunctions against well chosen observables. Of course, the high eigenvalue asymptotics is not simple. One would like to know the behavior as $\lambda_{j} \rightarrow \infty$ (or $\hbar \rightarrow 0$ of the diagonal matrix elements $\left\langle A \varphi_{j}, \varphi_{j}\right\rangle$ and the transition amplitudes $\left\langle A \varphi_{i}, \varphi_{j}\right\rangle$ between states for $A \in \Psi^{0}(M)$. One of the principal problems of quantum chaos is the following:

Problem 3.1. Determine the set $\mathcal{Q}$ of "quantum limits", i.e. weak* limit points of the sequence of invariant eigenfunction states $\left\{\rho_{k}\right\}$ or equivalently of the Wigner distributions $\left\{W_{k}\right\}$.

As will be illustrated below in simple examples, weak limits reflect the concentration and oscillation properties of eigenfunctions.

Off-diagonal matrix elements

$$
\rho_{j k}(A)=\left\langle A \varphi_{i}, \varphi_{j}\right\rangle
$$

are also important as transition amplitudes between states. They no longer define states since $\rho_{j k}(I)=0$, are no longer positive, and are no longer invariant. Indeed, $\rho_{j k}\left(U_{t} A U_{t}^{*}\right)=e^{i t\left(\lambda_{j}-\lambda_{k}\right)} \rho_{j k}(A)$, so they are eigenvectors of the automorphism $\alpha_{t}$ of (14). A sequence of such matrix elements cannot have a weak limit unless the spectral gap $\lambda_{j}-\lambda_{k}$ tends to a limit $\tau \in \mathbb{R}$. Problem 3.1 has the following extension to off-diagonal elements:

Problem 3.2. Determine the set $\mathcal{Q}_{\tau}$ of "quantum limits", i.e. weak* limit points of the sequence $\left\{W_{k j}\right\}$ of distributions on the classical phase space $S^{*} M$, defined by

$$
\int_{X} a d W_{k j}:=\left\langle O p(a) \varphi_{k}, \varphi_{j}\right\rangle
$$


where $\lambda_{j}-\lambda_{k}=\tau+o(1)$ and where $a \in C^{\infty}\left(S^{*} M\right)$, or equivalently of the functionals $\rho_{j k}$.

3.1. Simplest properties of quantum limits. The first is that $\left\langle K \varphi_{k}, \varphi_{k}\right\rangle \rightarrow 0$ for any compact operator $K$. Indeed, any orthonormal basis such as $\left\{\varphi_{k}\right\}$ tends to zero weakly in $L^{2}$. Hence $\left\{K \varphi_{k}\right\}$ tends to zero in $L^{2}$ for any compact operator and in particular the diagonal matrix elements tend to zero. It follows that for any $A \in \Psi^{0}(M)$, any limit of a sequence of $\left\langle A \varphi_{k}, \varphi_{k}\right\rangle$ is equally a limit of $\left\langle(A+K) \varphi_{k}, \varphi_{k}\right\rangle$.

Any two choices of $O p$, i.e. of quantizations of homogeneous symbols (of order zero) as pseudo-differential operators, are the same to leading order. Hence their difference is compact. Since a negative order pseudo-differential operator is compact, $\mathcal{Q}$ is independent of the definition of $O p$.

These properties do not use the fact that $\varphi_{j}$ are eigenfunctions. The next property of the $\rho_{k}$ are consequences of the fact $\rho_{k} \in \mathcal{E}_{\mathbb{R}}$.

Proposition 3.3. $\mathcal{Q} \subset \mathcal{M}_{I}$, where $\mathcal{M}_{I}$ is the convex set of invariant probability measures for the geodesic flow. They are also time-reversal invariant.

To see this, we first observe that any weak $*$ limit of of $\left\{\rho_{k}\right\}$ is a positive linear functional on symbols, which we identify with homogeneous functions of order zero on $T^{*} M$ or with smooth functions on $S^{*} M$. Indeed, any limit of $\left\langle A \varphi_{k}, \varphi_{k}\right\rangle$, is bounded by $\inf _{K}\|A+K\|$ (the infimum taken over compact operators), and for any $A \in \Psi^{0},\left\|\sigma_{A}\right\|_{L^{\infty}}=\inf _{K}\|A+K\|$. Hence any weak limit is bounded by a constant times $\left\|\sigma_{A}\right\|_{L^{\infty}}$ and is therefore continuous on $C\left(S^{*} M\right)$. It is a positive functional since each $\rho_{j}$ is and hence any limit is a probability measure. The invariance under $g^{t}$ follows from $\rho_{k} \in \mathcal{E}_{\mathbb{R}}$ by Egorov's theorem: any limit of $\rho_{k}(A)$ is a limit of $\rho_{k}\left(O p\left(\sigma_{A} \circ g^{t}\right)\right)$ and hence the limit measure is $g^{t}$ invariant. Furthermore, the limit measures are time-reversal invariant, i.e. invariant under $(x, \xi) \rightarrow(x,-\xi)$ since the eigenfunctions are real-valued.

Problem 3.1 is thus to identify which invariant measures in $\mathcal{M}_{I}$ show up as weak limits of the functionals $\rho_{k}$ or equivalently of the Wigner distributions $d W_{k}$. The problem is that $\mathcal{M}_{I}$ can be a very large set. Examples of invariant probability measures for the geodesic flow include:

(1) Normalized Liouville measure $d \mu_{L}$. In fact, the functional $\omega$ of (43) is also a state on $\Psi^{0}$ for the reason explained above. A subsequence $\left\{\varphi_{j_{k}}\right\}$ of eigenfunctions is considered diffuse if $\rho_{j_{k}} \rightarrow \omega$.

(2) A periodic orbit measure $\mu_{\gamma}$ defined by $\mu_{\gamma}(A)=\frac{1}{L_{\gamma}} \int_{\gamma} \sigma_{A} d s$ where $L_{\gamma}$ is the length of $\gamma$. A sequence of eigenfunctions for which $\rho_{k_{j}} \rightarrow \mu_{\gamma}$ obviously concentrates (or strongly "scars") on the closed geodesic.

(3) A finite convex combination $\sum_{j=1}^{M} c_{j} d \mu_{\gamma_{j}}$ of periodic orbit measures.

(4) A mixed measure such as $\frac{1}{2} d \mu_{L}+\frac{1}{2} \sum_{j=1}^{M} c_{j} d \mu_{\gamma_{j}}$ 
(5) A delta-function along an invariant Lagrangian manifold $\Lambda \subset S^{*} M$. The associated eigenfunctions are viewed as localizing along $\Lambda$.

(6) There are many additional kinds of singular measures in the Anosov case.

Thus, the constraint in the Proposition is far from enough to determine $\mathcal{Q}$.

To pin down $\mathcal{Q}$, it is necessary to find more constraints on the states $\rho_{k}$ and their weak* limits. We must use the quantum mechanics of $\rho_{k}$ to pin down the possible classical limits.

What possible additional constraints are there on the $\rho_{k}$ ? To date, only two are known.

- Further symmetries of the $\varphi_{j}$. In general there are none. But in special cases they exist. The most significant case is that of Hecke eigenfunctions, which carry an infinite number of further symmetries. See $\S 9$ and [LIND, Sound1].

- Entropies of limit measures. Lower bounds were obtained by N. Anantharaman and S. Nonnemacher $[\mathbf{A}, \mathbf{A N}]$ in the case of $(M, g)$ with Anosov geodesic flow (see also $[\mathbf{A N K}, \mathbf{R i v}]$.)

These constraints are of a very different nature. The Hecke symmetries are special to arithmetic hyperbolic quotients, where one supplement the geodesic flow-wave group connection with the Hecke symmetries and connections to number theory. The entropy bounds are very general and only use the geodesic flow-wave group connection.

3.2. Ergodic sequences of eigenfunctions. A subsequence $\left\{\varphi_{j_{k}}\right\}$ of eigenfunctions is called ergodic if the only weak $*$ limit of the sequence of $\rho_{j_{k}}$ is $d \mu_{L}$ or equivalently the Liouville state $\omega$. If $d W_{k_{j}} \rightarrow \omega$ then in particular, we have

$$
\frac{1}{\operatorname{Vol}(M)} \int_{E}\left|\varphi_{k_{j}}(x)\right|^{2} d \operatorname{Vol} \rightarrow \frac{\operatorname{Vol}(E)}{\operatorname{Vol}(M)}
$$

for any measurable set $E$ whose boundary has measure zero. In the interpretation of $\left|\varphi_{k_{j}}(x)\right|^{2} d V o l$ as the probability density of finding a particle of energy $\lambda_{k}^{2}$ at $x$, this says that the sequence of probabilities tends to uniform measure.

However, $W_{k_{j}} \rightarrow \omega$ is much stronger since it says that the eigenfunctions become diffuse on the energy surface $S^{*} M$ and not just on the configuration space $M$. One can quantize characteristic functions $\mathbf{1}_{E}$ of open sets in $S^{*} M$ whose boundaries have measure zero. Then

$$
\begin{aligned}
\left\langle O p\left(\mathbf{1}_{E}\right) \varphi_{j}, \varphi_{j}\right\rangle= & \text { the amplitude that the particle in energy state } \\
& \lambda_{j}^{2} \text { lies in E. }
\end{aligned}
$$


For an ergodic sequence of eigenfunctions,

$$
\left\langle O p\left(\mathbf{1}_{E}\right) \varphi_{j_{k}}, \varphi_{j_{k}}\right\rangle \rightarrow \frac{\mu_{L}(E)}{\mu_{L}\left(S^{*} M\right)},
$$

so that the particle becomes diffuse, i.e. uniformly distributed on $S^{*} M$. This is the quantum analogue of the property of uniform distribution of typical geodesics of ergodic geodesic flows (Birkhoff's ergodic theorem).

3.3. QUE. The Laplacian $\Delta$ or $(M, g)$ is said to be QUE (quantum uniquely ergodic) if $\mathcal{Q}=\left\{\mu_{L}\right\}$, i.e. the only quantum limit measure for any orthonormal basis of eigenfunctions is Liouville measure.

ConjeCture 3.4. (Rudnick-Sarnak, $[\mathbf{R S}]$ ) Let $(M, g)$ be a negatively curved manifold. Then $\Delta$ is QUE.

In $[\mathbf{R S}]$, the case of arithmetic manifolds is investigated and the role of the Hecke operators is clarified and exploited. In particular the arithmetic QUE Conjecture refers to the limits of Hecke eigen-states, i.e. eigenfunctions of the arithmetic symmetries called Hecke operators. As reviewed in $\S 9$, in this case the conjecture in all its forms is more or less completely solved. E. Lindenstrauss [LIND] (together with the recent final step in the noncompact case by Soundararajan [Sound1]) proves this arithmetic QUE for arithmetic surfaces. If the multiplicity of the eigenvalues are uniformly bounded then one can deduce full QUE from arithmetic QUE, i.e. QUE for any orthonormal basis of eigenfunctions (see $\S 9$ ). Such uniform bounds on multiplicities are however far out of the range of current technology . The analogue of arithmetic QUE for Hecek holomorphic forms on noncompact arithmetic surfaces has recently been settled by Holowinsky and Soundararajan $[\mathbf{H o l}, \mathbf{H S}]$. In this case, the Hecke condition cannot be dropped due to the high multiplicity of such forms. Their methods are entirely arithmetical and we won't discuss them further here.

Although we are not discussing quantum cat maps in detail, it should be emphasized that quantizations of hyperbolic (Anosov) symplectic maps of the torus) are not QUE. For a sparse sequence of Planck constants $\hbar_{k}$, there exist eigenfunctions of the quantum cat map which partly scar on a hyperbolic fixed point (see Faure-Nonnenmacher-de Bièvre $[$ FNB]). The multiplicities of the corresponding eigenvalues are of order $\hbar_{k}^{-1} /|\log \hbar|$. It is unknown if anything analogous can occur in the Riemannian setting, but as yet there is nothing to rule it out.

As pointed out in $[\mathbf{Z 1 0}]$, QUE would follow if one could prove that quantum limits were invariant under a uniquely ergodic flow such as the horocycle flow of a compact hyperbolic quotient. A problem in trying to use this approach is that the horocycle flow is not Hamiltonian with respect to the standard symplectic form on $T^{*} \mathbf{X}_{\Gamma}$, i.e. it cannot be quantized. It is however Hamiltonian with respect to a modified symplectic structure. The modified symplectic structure corresponds to letting the weight of automorphic forms vary with the $\Delta$ eigenvalue. As a result, one does get large 
sequences of automorphic forms which are Liouville distributed. But QUE in the standard sense refers to sequences with fixed weight. It turns out that the weight has to grow so quickly for the QUE sequence that one cannot seem to relate the QUE result to quantum limits of forms with fixed weight [Z10].

3.4. Simplest example: $S^{1}$. The only computable example of ergodic eigenfunctions is the sequence of normalized eigenfunctions $2 \sin k \pi x, 2 \cos k$ $\psi x$ on $S^{1}$. Note that they are real valued; the exponentials $e^{i k x}$ are not quantum ergodic.

The energy surface of $T^{*} S^{1}=S^{1} \times \mathbb{R}_{\xi}$ is the pair of circles $|\xi|= \pm 1$. The geodesic flow has two invariant sets of positive measure (the two components), but the flow is time-reversal invariant under $(x, \xi) \rightarrow(x-\xi)$ and the quotient flow is ergodic. The real eigenfunctions are invariant under complex conjugation and therefore the quotient quantum system is ergodic.

Quantum ergodicity in this case amounts to

$$
\frac{1}{\pi} \int_{0}^{2 \pi} V(x)(\sin k x)^{2} d x \rightarrow \frac{1}{2 \pi} \int_{0}^{2 \pi} V d x .
$$

This is obvious by writing $\sin k x$ in terms of exponentials and using the Riemann-Lebesgue Lemma.

This simple example illustrates an important aspect of quantum limits: They are weak limits which owe to the fast oscillation of the eigenfunction squares. In the limit the oscillating functions tend to their mean values in the weak sense.

In particular, it illustrates why we only consider squares and not other powers of eigenfunctions: weak limits are not preserved under non-linear functionals such as powers. The study of $L^{p}$ norms of chaotic eigenfunctions is very difficult (there are Iwaniec-Sarnak for Hecke eigenfunctions).

3.5. Example of quantum limits: the flat torus. The only examples where one can compute quantum limits directly are the completely integrable ones such as the standard sphere, torus or symmetric spaces. These examples of course lie at the opposite extreme from chaotic or ergodic dynamics. We use the simplest one, the flat torus $\mathbb{R}^{n} / \mathbb{Z}^{n}$, to illustrate the definition of weak* limits.

An orthonormal basis of eigenfunctions is furnished by the standard exponentials $e^{2 \pi i\langle k, x\rangle}$ with $k \in \mathbb{Z}^{n}$. Obviously, $\left|e^{2 \pi i\langle k, x\rangle}\right|^{2}=1$, so the eigenfunctions are diffuse in configuration space. But they are far from diffuse in phase space. For any pseudodifferential operator, $A e^{2 \pi i\langle k, x\rangle}=a(x, k) e^{2 \pi i\langle k, x\rangle}$ where $a(x, k)$ is the complete symbol. Thus,

$$
\left\langle A e^{2 \pi i\langle k, x\rangle}, e^{2 \pi i\langle k, x\rangle}\right\rangle=\int_{\mathbb{R}^{n} / \mathbb{Z}^{n}} a(x, k) d x \sim \int_{\mathbb{R}^{n} / \mathbb{Z}^{n}} \sigma_{A}\left(x, \frac{k}{|k|}\right) d x .
$$


Thus, the Wigner distribution is $\delta_{\xi-k}$. A subsequence $e^{2 \pi i\left\langle k_{j}, x\right\rangle}$ of eigenfunctions has a weak limit if and only if $\frac{k_{j}}{\left|k_{j}\right|}$ tends to a limit vector $\xi_{0}$ in the unit sphere in $\mathbb{R}^{n}$. In this case, the associated weak ${ }^{*}$ limit is $\int_{\mathbb{R}^{n} / \mathbb{Z}^{n}} \sigma_{A}\left(x, \xi_{0}\right) d x$, i.e. the delta-function on the invariant torus $T_{\xi_{0}} \subset S^{*} M$ defined by the constant momentum condition $\xi=\xi_{0}$. The eigenfunctions are said to localize under this invariant torus for $g^{t}$.

The invariant torus is a Lagrangian submanifold of $T^{*} \mathbb{R}^{n} / \mathbb{Z}^{n}$, i.e. a submanifold of dimension $n$ on which the standard symplectic form $d x \wedge d \xi$ restricts to zero. The exponentials are special cases of WKB or Lagrangian states $a_{\hbar} e^{\frac{i}{\hbar} S}$, where $a_{\hbar}$ is a semi-classical symbol $a_{\hbar} \sim a_{j} \hbar^{j}$. The associated Lagrangian submanifold is the graph $(x, d S(x))$ of $S$. Thus, $\langle k, x\rangle$ generates the Lagrangian submanifold $\xi=\frac{k}{|k|}$.

In general, one says that a sequence $\left\{\varphi_{j_{k}}\right\}$ of eigenfunctions concentrates microlocally on a Lagrangian submanifold $\Lambda \subset S^{*} M$ if

$$
\left\langle A \varphi_{j_{k}}, \varphi_{j_{k}}\right\rangle \rightarrow \int_{\Lambda} \sigma_{A} d \nu
$$

for some probability measure $\nu$ on $\Lambda$. Necessarily, $\Lambda$ is invariant under $g^{t}$.

3.6. Hyperbolic case. Using the Helgason Poisson integral formula, the Wigner distributions can be expressed in terms of the $d T_{i r_{h}}$ and $e_{i r_{j}}$ as follows. As in $[\mathbf{Z 3}]$, we define the hyperbolic calculus of pseudo-differential operators $O p(a)$ on $\mathbf{D}$ by

$$
O p(a) e^{\left(\frac{1}{2}+i r\right)\langle z, b\rangle}=a(z, b, r) e^{\left(\frac{1}{2}+i r\right)\langle z, b\rangle} .
$$

We assume that the complete symbol $a$ is a polyhomogeneous function of $r$ in the classical sense that

$$
a(z, b, r) \sim \sum_{j=0}^{\infty} a_{j}(z, b) r^{-j+m}
$$

for some $m$ (called its order). By asymptotics is meant that

$$
a(z, b, r)-\sum_{j=0}^{R} a_{j}(z, b) r^{-j+m} \in S^{m-R-1}
$$

where $\sigma \in S^{k}$ if $\sup (1+r)^{j-k}\left|D_{z}^{\alpha} D_{b}^{\beta} D_{r}^{j} \sigma(z, b, r)\right|<+\infty$ for all compact set and for all $\alpha, \beta, j$.

The non-Euclidean Fourier inversion formula then extends the definition of $O p(a)$ to $C_{c}^{\infty}(\mathbf{D})$ :

$$
O p(a) u(z)=\int_{B} \int_{\mathbb{R}} a(z, b, r) e^{\left(\frac{1}{2}+i r\right)\langle z, b\rangle} \mathcal{F} u(r, b) r \tanh (2 \pi r) d r|d b| .
$$

A key property of $O p$ is that $O p(a)$ commutes with the action of an element $\gamma \in G\left(T_{\gamma} u(z)=u(\gamma z)\right)$ if and only if $a(\gamma z, \gamma b, r)=a(z, b, r)$. 
$\Gamma$-equivariant pseudodifferential operators then define operators on the quotient $\mathbf{X}_{\Gamma}$.

By the Helgason-Poisson formula one has a relative explicit formula for the Wigner distributions $\left.W_{i r_{j}} \in \mathcal{D}^{\prime}\left(S^{*} \mathbf{X}_{\Gamma}\right)\right)$ defined by

$\left\langle a, W_{i r_{j}}\right\rangle=\int_{S^{*} \mathbf{X}_{\Gamma}} a(g) W_{i r_{j}}(d g):=\left\langle O p(a) \varphi_{i r_{j}}, \varphi_{i r_{j}}\right\rangle_{L^{2}\left(\mathbf{X}_{\Gamma}\right)}, \quad a \in C^{\infty}\left(S^{*} \mathbf{X}_{\Gamma}\right)$.

Equivalently we have

$$
W_{i r_{j}}=\varphi_{i r_{j}} e_{i r_{j}}
$$

Thus, the Wigner distributions are far more diffuse in phase space than in the case of the flat torus. But this does not rule out that their weak* limits could have a singular concentration.

3.6.1. Patterson-Sullivan distributions. Egorov's theorem implies that Wigner distributions tend to invariant measures for the geodesic flow. The question arises whether there exist $g^{t}$-invariant distributions constructed from eigenfunctions which are asymptotic to the Wigner distributions. In [AZ] such distributions were constructed on hyperbolic surfaces and termed "Patterson-Sullivan distributions" by analogy with their construction of boundary measures associated to ground states on infinite volume hyperbolic manifolds.

Definition: The Patterson-Sullivan distribution associated to a real eigenfunction $\varphi_{i r_{j}}$ is the distribution on $B \times B \backslash \Delta$ defined by

$$
p s_{i r_{j}}\left(d b^{\prime}, d b\right):=\frac{T_{i r_{j}}(d b) T_{i r_{j}}\left(d b^{\prime}\right)}{\left|b-b^{\prime}\right|^{1+2 i r_{j}}}
$$

If $\varphi_{i r_{j}}$ is $\Gamma$-automorphic, then $p s_{i r_{j}}\left(d b^{\prime}, d b\right)$ is $\Gamma$-invariant and time reversal invariant.

To obtain a $g^{t}$-invariant distribution on $S^{*} \mathbf{X}_{\Gamma}$, we tensor $p s_{i r_{j}}$ with $d t$. We then normalize by dividing by the integral against 1 . The result is an invariant distribution $\hat{P S}{ }_{i r_{j}}$ for $g^{t}$ constructed as a quadratic expression in the eigenfunctions. In $[\mathbf{A Z}]$ Theorem 1.1, it is proved (theorem that

$$
\int_{S^{*} \mathbf{X}_{\Gamma}} a \hat{P S} i_{i r_{j}}=\int_{S^{*} \mathbf{X}_{\Gamma}} a W_{i r_{j}}+O\left(r_{j}^{-1}\right)
$$

Hence the quantum limits problem is equally one of determining the weak* limits of the Patterson-Sullivan distributions. It is shown in $[\mathbf{A Z}]$ that they are residues of dynamical L-functions and hence have a purely classical definition.

In fact, there is an explicit intertwining operator $L_{r_{j}}$ mapping $P S_{i r_{j}} \rightarrow$ $W_{i r_{j}}$ and we have

$$
\left\langle a, W_{i r_{j}}\right\rangle=\left\langle a, \hat{P S} S_{i r_{j}}\right\rangle+r_{j}^{-1}\left\langle L_{2}(a), \hat{P S_{i r_{j}}}\right\rangle+O\left(r_{j}^{-2}\right) .
$$




\section{Quantum ergodicity and mixing of eigenfunctions}

In this section, we review a basic result on quantum ergodicity. We assume that the geodesic flow of $(M, g)$ is ergodic. Ergodicity of $g^{t}$ means that Liouville measure $d \mu_{L}$ is an ergodic measure for $g^{t}$ on $S^{*} M$, i.e. an extreme point of $\mathcal{M}_{I}$. That is, there any $g^{t}$-invariant set has Liouville measure zero or one. Ergodicity is a spectral property of the operator $V^{t}$ of (15) on $L^{2}\left(S^{*} M, d \mu_{L}\right)$, namely that $V^{t}$ has 1 as an eigenvalue of multiplicity one. That is, the only invariant $L^{2}$ functions (with respect to Liouville measure) are the constant functions.

In this case, there is a general result which originated in the work of A. I. Schnirelman and was developed into the following theorem by S. Zelditch, Y. Colin de Verdière on manifolds without boundary and by P. Gérard-E. Leichtnam and S. Zelditch-M. Zworski on manifolds with boundary.

THEOREM 4.1. Let $(M, g)$ be a compact Riemannian manifold (possibly with boundary), and let $\left\{\lambda_{j}, \varphi_{j}\right\}$ be the spectral data of its Laplacian $\Delta$. Then the geodesic flow $G^{t}$ is ergodic on $\left(S^{*} M, d \mu_{L}\right)$ if and only if, for every $A \in \Psi^{o}(M)$, we have:

- (i) $\lim _{\lambda \rightarrow \infty} \frac{1}{N(\lambda)} \sum_{\lambda_{j} \leq \lambda}\left|\left(A \varphi_{j}, \varphi_{j}\right)-\omega(A)\right|^{2}=0$.

- $\left(\right.$ ii) $(\forall \epsilon)(\exists \delta) \lim \sup _{\lambda \rightarrow \infty} \frac{1}{N(\lambda)} \sum_{\substack{j \neq k: \lambda_{j}, \lambda_{k} \leq \lambda \\\left|\lambda_{j}-\lambda_{k}\right|<\delta}}\left|\left(A \varphi_{j}, \varphi_{k}\right)\right|^{2}<\epsilon$

The diagonal result may be interpreted as a variance result for the local Weyl law. Since all the terms are positive, the asymptotic is equivalent to the existence of a s subsequence $\left\{\varphi_{j_{k}}\right\}$ of eigenfunctions whose indices $j_{k}$ have counting density one for which $\left\langle A \varphi_{j_{k}}, \varphi_{j_{k}}\right\rangle \rightarrow \omega(A)$ for any $A \in \Psi^{0}(M)$. As above, such a sequence of eigenfunctions is called ergodic. One can sharpen the results by averaging over eigenvalues in the shorter interval $[\lambda, \lambda+1]$ rather than in $[0, \lambda]$.

The off-diagonal statement was proved in $[\mathbf{Z 9}]$ and the fact that its proof can be reversed to prove the converse direction was observed by Sunada in $[\mathbf{S u}]$. A generalization to finite area hyperbolic surfaces is in $[\mathbf{Z 8}]$.

The first statement (i) is essentially a convexity result. It remains true if one replaces the square by any convex function $F$ on the spectrum of $A$,

$$
\frac{1}{N(E)} \sum_{\lambda_{j} \leq E} F\left(\left\langle A \varphi_{k}, \varphi_{k}\right\rangle-\omega(A)\right) \rightarrow 0 .
$$

4.1. Quantum ergodicity in terms of operator time and space averages. The diagonal variance asymptotics may be interpreted as a relation between operator time and space averages.

Definition: Let $A \in \Psi^{0}$ be an observable and define its time average to be:

$$
\langle A\rangle:=\lim _{T \rightarrow \infty}\langle A\rangle_{T}
$$


where

$$
\langle A\rangle_{T}:=\frac{1}{2 T} \int_{-T}^{T} U^{t} A U^{-t} d t
$$

and its space average to be scalar operator

$$
\omega(A) \cdot I
$$

Then Theorem 4.1 (1) is (almost) equivalent to,

$$
\langle A\rangle=\omega(A) I+K, \quad \text { where } \lim _{\lambda \rightarrow \infty} \omega_{\lambda}\left(K^{*} K\right) \rightarrow 0,
$$

where $\omega_{\lambda}(A)=\frac{1}{N(\lambda)} \operatorname{Tr} E(\lambda) A$. Thus, the time average equals the space average plus a term $K$ which is semi-classically small in the sense that its Hilbert-Schmidt norm square $\left\|E_{\lambda} K\right\|_{H S}^{2}$ in the span of the eigenfunctions of eigenvalue $\leq \lambda$ is $o(N(\lambda))$.

This is not exactly equivalent to Theorem 4.1 (1) since it is independent of the choice of orthonormal basis, while the previous result depends on the choice of basis. However, when all eigenvalues have multiplicity one, then the two are equivalent. To see the equivalence, note that $\langle A\rangle$ commutes with $\sqrt{\Delta}$ and hence is diagonal in the basis $\left\{\varphi_{j}\right\}$ of joint eigenfunctions of $\langle A\rangle$ and of $U_{t}$. Hence $K$ is the diagonal matrix with entries $\left\langle A \varphi_{k}, \varphi_{k}\right\rangle-\omega(A)$. The condition is therefore equivalent to

$$
\lim _{E \rightarrow \infty} \frac{1}{N(E)} \sum_{\lambda_{j} \leq E}\left|\left\langle A \varphi_{k}, \varphi_{k}\right\rangle-\omega(A)\right|^{2}=0 .
$$

4.2. Heuristic proof of Theorem 4.1 (i). There is a simple picture of eigenfunction states which makes Theorem 4.1 seem obvious. Justifying the picture is more difficult than the formal proof below but the reader may find it illuminating and convincing.

First, one should re-formulate the ergodicity of $g^{t}$ as a property of the Liouville measure $d \mu_{L}$ : ergodicity is equivalent to the statement $d \mu_{L}$ is an extreme point of the compact convex set $\mathcal{M}_{I}$. Moreover, it implies that the Liouville state $\omega$ on $\Psi^{0}(M)$ is an extreme point of the compact convex set $\mathcal{E}_{\mathbb{R}}$ of invariant states for $\alpha_{t}$ of (14); see [Ru] for background. But the local Weyl law says that $\omega$ is also the limit of the convex combination $\frac{1}{N(E)} \sum_{\lambda_{j} \leq E} \rho_{j}$. An extreme point cannot be written as a convex combination of other states unless all the states in the combination are equal to it. In our case, $\omega$ is only a limit of convex combinations so it need not (and does not) equal each term. However, almost all terms in the sequence must tend to $\omega$, and that is equivalent to (1).

One could make this argument rigorous by considering whether Liouville measure is an exposed point of $\mathcal{E}_{I}$ and $\mathcal{M}_{I}$. Namely, is there a linear functional $\Lambda$ which is equal to zero at $\omega$ and is $<0$ everywhere else on $\mathcal{E}_{I}$ ? If so, the fact that $\frac{1}{N(E)} \sum_{\lambda_{j} \leq E} \Lambda\left(\rho_{j}\right) \rightarrow 0$ implies that $\Lambda\left(\rho_{j}\right) \rightarrow 0$ for a subsequence of density one. For one gets an obvious contradiction if 
$\Lambda\left(\rho_{j_{k}}\right) \leq-\epsilon<0$ for some $\epsilon>0$ and a subsequence of positive density. But then $\rho_{j_{k}} \rightarrow \omega$ since $\omega$ is the unique state with $\Lambda(\rho)=0$.

In $[\mathbf{J}]$ it is proved that Liouville measure (or any ergodic measure) is exposed in $\mathcal{M}_{I}$. It is stated in the following form: For any ergodic invariant probability measure $\mu$, there exists a continuous function $f$ on $S^{*} M$ so that $\mu$ is the unique $f$-maximizing measure in the sense that

$$
\int f d \mu=\sup \left\{\int f d m: m \in \mathcal{M}_{I}\right\} .
$$

To complete the proof, one would need to show that the extreme point $\omega$ is exposed in $\mathcal{E}_{I}$ for the $\mathrm{C}^{*}$ algebra defined by the norm-closure of $\Psi^{0}(M)$.

4.3. Sketch of proof of Theorem 4.1 (i). We now sketch the proof of (52). By time averaging, we have

$$
\sum_{\lambda_{j} \leq E} F\left(\left\langle A \varphi_{k}, \varphi_{k}\right\rangle-\omega(A)\right)=\sum_{\lambda_{j} \leq E} F\left(\left\langle\langle A\rangle_{T}-\omega(A) \varphi_{k}, \varphi_{k}\right\rangle\right) .
$$

We then apply the Peierls-Bogoliubov inequality

$$
\sum_{j=1}^{n} F\left(\left(B \varphi_{j}, \varphi_{j}\right)\right) \leq \operatorname{Tr} F(B)
$$

with $B=\Pi_{E}\left[\langle A\rangle_{T}-\omega(A)\right] \Pi_{E}$ to get:

$$
\sum_{\lambda_{j} \leq E} F\left(\left\langle\langle A\rangle_{T}-\omega(A) \varphi_{k}, \varphi_{k}\right\rangle\right) \leq \operatorname{Tr} F\left(\Pi_{E}\left[\langle A\rangle_{T}-\omega(A)\right] \Pi_{E}\right) .
$$

Here, $\Pi_{E}$ is the spectral projection for $\hat{H}$ corresponding to the interval $[0, E]$. By the Berezin inequality (if $F(0)=0$ ):

$$
\begin{aligned}
\frac{1}{N(E)} \operatorname{Tr} F\left(\Pi_{E}\left[\langle A\rangle_{T}-\omega(A)\right] \Pi_{E}\right) & \leq \frac{1}{N(E)} \operatorname{Tr} \Pi_{E} F\left(\left[\langle A\rangle_{T}-\omega(A)\right]\right) \Pi_{E} \\
& =\omega_{E}\left(\varphi\left(\langle A\rangle_{T}-\omega(A)\right)\right) .
\end{aligned}
$$

As long as $F$ is smooth, $F\left(\langle A\rangle_{T}-\omega(A)\right)$ is a pseudodifferential operator of order zero with principal symbol $F\left(\left\langle\sigma_{A}\right\rangle_{T}-\omega(A)\right)$. By the assumption that $\omega_{E} \rightarrow \omega$ we get

$$
\lim _{E \rightarrow \infty} \frac{1}{N(E)} \sum_{\lambda_{j} \leq E} F\left(\left\langle A \varphi_{k}, \varphi_{k}\right\rangle-\omega(A)\right) \leq \int_{\{H=1\}} F\left(\left\langle\sigma_{A}\right\rangle_{T}-\omega(A)\right) d \mu_{L} .
$$

As $T \rightarrow \infty$ the right side approaches $\varphi(0)$ by the dominated convergence theorem and by Birkhoff's ergodic theorem. Since the left hand side is independent of $T$, this implies that

$$
\lim _{E \rightarrow \infty} \frac{1}{N(E)} \sum_{\lambda_{j} \leq E} F\left(\left\langle A \varphi_{k}, \varphi_{k}\right\rangle-\omega(A)\right)=0
$$

for any smooth convex $F$ on $\operatorname{Spec}(A)$ with $F(0)=0$. 
This proof can only be used directly for scalar Laplacians on manifolds without boundary, but it still works as a template in more involved situations. For instance, on manifolds with boundary, conjugation by the wave group is not a true automorphism of the observable algebra. In quantum ergodic restriction theorems (see $\S 6$ ), the appropriate conjugation is an endomorphism but not an automorphism. Or when $\Delta$ has continuous spectrum (as in finite area hyperbolic surfaces), one must adapt the proof to states which are not $L^{2}$-normalized [Z8].

4.4. QUE in terms of time and space averages. The quantum unique ergodicity problem (the term is due to Rudnick-Sarnak $[\mathbf{R S}]$ ) is the following:

Problem 4.2. Suppose the geodesic flow $g^{t}$ of $(M, g)$ is ergodic on $S^{*} M$. Is the operator $K$ in

$$
\langle A\rangle=\omega(A)+K
$$

a compact operator? Equivalently is $\mathcal{Q}=\left\{d \mu_{L}\right\}$ ? In this case, $\sqrt{\Delta}$ is said to be QUE (quantum uniquely ergodic)

Compactness of $K$ implies that $\left\langle K \varphi_{k}, \varphi_{k}\right\rangle \rightarrow 0$, hence $\left\langle A \varphi_{k}, \varphi_{k}\right\rangle \rightarrow \omega(A)$ along the entire sequence.

Rudnick-Sarnak conjectured that $\Delta$ of negatively curved manifolds are QUE, i.e. that for any orthonormal basis of eigenfunctions, the Liouville measure is the only quantum limit $[\mathbf{R S}]$.

4.5. Converse QE. So far we have not mentioned Theorem 4.1 (2). An interesting open problem is the extent to which (2) is actually necessary for the equivalence to classical ergodicity.

PRoblem 4.3. Suppose that $\sqrt{\Delta}$ is quantum ergodic in the sense that (1) holds in Theorem 4.1. What are the properties of the geodesic flow $g^{t}$. Is it ergodic (in the generic case)?

In the larger class of Schrödinger operators, there is a simple example of a Hamiltonian system which is quantum ergodic but not classically ergodic: namely, a Schrödinger operator with a symmetric double well potential $W$. That is, $W$ is a $W$ shaped potential with two wells and a $\mathbb{Z}_{2}$ symmetry exchanging the wells. The low energy levels consist of two connected components interchanged by the symmetry, and hence the classical Hamiltonian flow is not ergodic. However, all eigenfunctions of the Schrödinger operator $-\frac{d^{2}}{d x^{2}}+W$ are either even or odd and thus have the same mass in both wells. It is easy to see that the quantum Hamiltonian is quantum ergodic.

Recently, B. Gutkin [Gut] has given a two dimensional example of a domain with boundary which is quantum ergodic but not classically ergodic and which is a two dimensional analogue of a double well potential. The domain is a so-called hippodrome (race-track) stadium. Similarly to the double well potential, there are two invariant sets interchanged by a $\mathbb{Z}_{2}$ 
symmetry. They correspond to the two orientations with which the race could occur. Hence the classical billiard flow on the domain is not ergodic. After dividing by the $\mathbb{Z}^{2}$ symmetry the hippodrome has ergodic billiards, hence by Theorem 4.1, the quotient domain is quantum ergodic. But the The eigenfunctions are again either even or odd. Hence the hippodrome is quantum ergodic but not classically ergodic.

Little is known about converse quantum ergodicity in the abscence of symmetry. It is known that if there exists an open set in $S^{*} M$ filled by periodic orbits, then the Laplacian cannot be quantum ergodic (see [MOZ] for recent results and references). But it is not even known at this time whether KAM systems, which have Cantor-like invariant sets of positive measure, are not quantum ergodic. It is known that there exist a positive proportion of approximate eigenfunctions (quasi-modes) which localize on the invariant tori, but it has not been proved that a positive proportion of actual eigenfunctions have this localization property.

4.6. Quantum weak mixing. There are parallel results on quantizations of weak-mixing geodesic flows. We recall that the geodesic flow of $(M, g)$ is weak mixing if the operator $V^{t}$ has purely continuous spectrum on the orthogonal complement of the constant functions in $L^{2}\left(S^{*} M, d \mu_{L}\right)$.

THEOREM 4.4. [Z8] The geodesic flow $g^{t}$ of $(M, g)$ is weak mixing if and only if the conditions (1)-(2) of Theorem 4.1 hold and additionally, for any $A \in \Psi^{o}(M)$,

$$
(\forall \epsilon)(\exists \delta) \limsup _{\lambda \rightarrow \infty} \frac{1}{N(\lambda)} \sum_{\substack{j \neq k: \lambda_{j}, \lambda_{k} \leq \lambda \\\left|\lambda_{j}-\lambda_{k}-\tau\right|<\delta}}\left|\left(A \varphi_{j}, \varphi_{k}\right)\right|^{2}<\epsilon \quad(\forall \tau \in \mathbb{R})
$$

The restriction $j \neq k$ is of course redundant unless $\tau=0$, in which case the statement coincides with quantum ergodicity. This result follows from the general asymptotic formula, valid for any compact Riemannian manifold $(M, g)$, that

$$
\begin{gathered}
\frac{1}{N(\lambda)} \sum_{i \neq j, \lambda_{i}, \lambda_{j} \leq \lambda}\left|\left\langle A \varphi_{i}, \varphi_{j}\right\rangle\right|^{2}\left|\frac{\sin T\left(\lambda_{i}-\lambda_{j}-\tau\right)}{T\left(\lambda_{i}-\lambda_{j}-\tau\right)}\right|^{2} \\
\sim\left\|\frac{1}{2 T} \int_{-T}^{T} e^{i t \tau} V_{t}\left(\sigma_{A}\right)\right\|_{2}^{2}-\left|\frac{\sin T \tau}{T \tau}\right|^{2} \omega(A)^{2} .
\end{gathered}
$$

In the case of weak-mixing geodesic flows, the right hand side $\rightarrow 0$ as $T \rightarrow \infty$. As with diagonal sums, the sharper result is true where one averages over the short intervals $[\lambda, \lambda+1]$.

Theorem 4.4 is based on expressing the spectral measures of the geodesic flow in terms of matrix elements. The main limit formula is:

$$
\int_{\tau-\varepsilon}^{\tau+\varepsilon} d \mu_{\sigma_{A}}:=\lim _{\lambda \rightarrow \infty} \frac{1}{N(\lambda)} \sum_{i, j: \lambda_{j} \leq \lambda,\left|\lambda_{i}-\lambda_{j}-\tau\right|<\varepsilon}\left|\left\langle A \varphi_{i}, \varphi_{j}\right\rangle\right|^{2},
$$


where $d \mu_{\sigma_{A}}$ is the spectral measure for the geodesic flow corresponding to the principal symbol of $A, \sigma_{A} \in C^{\infty}\left(S^{*} M, d \mu_{L}\right)$. Recall that the spectral measure of $V^{t}$ corresponding to $f \in L^{2}$ is the measure $d \mu_{f}$ defined by

$$
\left\langle V^{t} f, f\right\rangle_{L^{2}\left(S^{*} M\right)}=\int_{\mathbb{R}} e^{\mathrm{it} \tau} d \mu_{f}(\tau) .
$$

4.7. Evolution of Lagrangian states. In this section, we briefly review results on evolution of Lagrangian states and coherent states. We follow in particular the article of R. Schubert [Schu3].

A simple Lagrangian or WKB state has the form $\psi_{\hbar}(x)=a(\hbar, x) e^{\frac{i}{\hbar} S(x)}$ where $a(\hbar, x)$ is a semi-classical symbol $a \sim \sum_{j=0}^{\infty} \hbar^{j} a_{j}(x)$. The phase $S$ generates the Lagrangian submanifold $(x, d S(x)) \subset T^{*} M$.

It is proved in Theorem 1 of [Schu3] that if $g^{t}$ is Anosov and if $\Lambda$ is transversal to the stable foliation $W^{s}$ (except on a set of codimension one), then there exists $C, \tau>0$ so that for every smooth density on $\Lambda$ and every smooth function $a \in C^{\infty}\left(S^{*} M\right)$, the Lagrangian state $\psi$ with symbol $\sigma_{\psi}$ satisfies,

$$
\left.\left|\left\langle U^{t} \psi, A U^{t} \psi\right\rangle-\int_{S^{*} M} \sigma_{A} d \mu_{L} \int_{\Lambda}\right| \sigma_{\psi}\right|^{2} \mid \leq C h e^{\Gamma|t|}+c e^{-t \tau} .
$$

In order that the right side tends to zero as $\hbar \rightarrow 0, t \rightarrow \infty$ it is necessary and sufficient that

$$
t \leq \frac{1-\epsilon}{\Gamma}|\log \hbar| .
$$

\section{Concentration of eigenfunctions around hyperbolic closed geodesics}

As mentioned above, the quantum ergodicity Theorem 4.1 leaves open the possible existence of a sparse (zero density) subsequence of eigenfunctions which "weakly scar" on a hyperbolic orbit $\gamma$ in the sense that its quantum limit $\nu_{0}$ contains a non-trivial multiple of the periodic orbit measure $c \mu_{\gamma}$ as a non-zero ergodic component. The Anantharaman entropy bound shows that when $(M, g)$ is Anosov, there cannot exist such a sequence of eigenfunctions (or even quasi-modes) which tend to $\mu_{\gamma}$ itself, but a quantum limit could have the form $c_{1} \mu_{\gamma}+c_{2} \mu_{L}$ for certain $c_{1}, c_{2}$ satisfying $c_{1}+c_{2}=1$. The question we address in this section is the possible mass profile of such a scarring eigenfunction in a neighborhood of $\gamma$. More precisely, how much mass does $\varphi_{\lambda}$ have in a shrinking $\hbar^{1 / 2-\epsilon}$ tube around a hyperbolic closed geodesic? This question will surface again in $\S 11.21$.

We first note that the existence of a quantum limit of the form $c_{1} \mu_{\gamma}+$ $c_{2} \mu_{L}$ for $(M, g)$ with Anosov geodesic flow is not so implausible. Analogous eigenfunction sequences do exist for the so-called quantum cat map [FNB, FN]. And exceptional sequences "scarring" on a certain 1-parameter family of periodic orbits exists for the Bunimovich stadium [Has]. At this time, there are no known examples of sequences of eigenfunctions or quasi-modes 
for $(M, g)$ with ergodic geodesic flow that "weakly scar" along a hyperbolic closed orbit $\gamma$ and no results prohibiting them.

For simplicity, assume that $(M, g)$ is a Riemannian manifold of dimension 2 with a closed geodesic $\gamma$ of length $L$. We assume $\gamma$ is an embedded (non self-intersecting) curve. If $\varphi_{j}$ is a Laplace eigenfunction, we define the mass profile of $\varphi_{j}$ near $\gamma$ to be the function

$$
M\left(\varphi_{j}\right)(r)=\int_{d(x, \gamma)=r}\left|\varphi_{j}\right|^{2} d S,
$$

where $d S \wedge d r=d V$.

Before considering possible mass profiles of eigenfunctions scarring on hyperbolic closed geodesics, let us recall the opposite and much better known case of scarring of Gaussian beams along elliptic closed geodesics on surfaces [Ra, Ra2, B.B]. When $\gamma$ is an elliptic closed geodesic, then there always exists a sequence of quasi-modes (Gaussian beams) which concentrates on $\gamma$. As the name suggests, Gaussian beams $\psi_{\lambda}$ oscillate like $e^{i \lambda s}$ along $\gamma$ and resemble Gaussians $\sqrt{\lambda} e^{-\lambda\langle A(s) y, y\rangle}$ (for some positive symmetric matrix $A(s))$ in the transverse direction with height $\lambda^{1 / 4}$ and concentrated in a $\frac{1}{\sqrt{\lambda}}$ tube around $\gamma$. Thus, the mass profile is a Gaussian probability measure with mean zero and variance $\lambda^{-1 / 2}$. The local model for such quasi-modes is that of a harmonic oscillator in the fibers of $N_{\gamma}$. One can construct the quasi-mode so that it is of infinite order. Of course, stable elliptic orbits do not exist when $(M, g)$ has ergodic geodesic flow (by the KAM theorem).

Now consider hyperbolic closed orbits. It was pointed out by Duistermaat [Dui] (Section 1.5) that one cannot construct analogous quasi-modes associated to hyperbolic closed geodesics as Lagrangian states. The stable/ unstable manifolds $\Lambda_{ \pm}$of $\gamma$, containing the geodesics which spiral in towards $\gamma$, are invariant Lagrangian submanifolds, but the only invariant half-density on the Lagrangians is the "delta"-density on the closed geodesic.

Further, there are apriori limitations on the degree to which eigenfunctions sequences can concentrate around hyperbolic closed geodesic on any $(M, g)$.

Theorem 5.1. [BZ, Chr, CVP] Let $(M, g)$ be a compact Riemannian manifold, and let $\gamma$ be a hyperbolic closed geodesic. Let $U$ be any tubular neighborhood of $\gamma$ in $M$. Then for any eigenfunction $\varphi_{\lambda}$, there exists a constant $C$ depending only on $U$ such that

$$
\int_{M \backslash U}\left|\varphi_{\lambda}\right|^{2} d V_{g} \geq \frac{C}{\log \lambda}\left\|\varphi_{\lambda}\right\|_{L^{2}}^{2} .
$$

More generally, let $A \in \Psi^{0}(M)$ be a pseudo-differential orbit whose symbol equals one in a neighborhood of $\gamma$ in $S_{g}^{*} M$ and equals zero outside another neighborhood. Then for any eigenfunction $\varphi_{\lambda}\left\|(I-A) \varphi_{\lambda}\right\|_{L^{2}} \geq$ $\frac{C}{\sqrt{\log \lambda}}\left\|\varphi_{\lambda}\right\|_{L^{2}}$. 
This allows scarring sequences along a hyperbolic orbit to occur, it just limits the rate at which the mass concentrates near $\gamma$. It implies that the mass profile of a sequence of eigenfunctions concentrating on a hyperbolic close geodesic has "long tails", i.e. there is a fairly large amount of mass far away from the geodesic, although sequences with the quantum limit $\mu_{\gamma}$ must tend to zero outside of any tube around the closed geodesic. Note that this result makes no dynamical hypotheses. It applies equally to $(M, g)$ with integrable geodesic flow and to those with Anosov geodesic flow. To the author's knowledge, there do not exist more precise results in the Anosov case.

An obvious question at this point is whether there are any examples of $(M, g)$, with any type of geodesic flow, possessing a sequence of eigenfunctions scarring on a closed hyperbolic orbit. The answer to this question is "yes". It is simple to see that such eigenfunctions exist in the opposite extreme of completely integrable systems, for instances surfaces of revolution like peanuts with hyperbolic waists. Examples include joint eigenfunctions of the square root of the Laplacian and rotation on surfaces of revolution with a hyperbolic waist. A truncated hyperbolic cylinder is another example studied in $[\mathbf{C V P}]$. In this case, the joint spectrum fills out the image of the moment map $\left(p_{\theta},|\xi|\right): T^{*} M \rightarrow \mathbb{R}^{2}$, where $p_{\theta}(x, \xi)=\left\langle\xi, \frac{\partial}{\partial \theta}\right\rangle$ is the angular momentum. At critical distances to the axis of rotation, the lattitude circles are closed geodesics and the level set of the moment map becomes singular. If the surface is shaped like a peanut, the waist is a hyperbolic closed geodesic. Joint eigenfunctions whose joint eigenvalues are asymptotic to the singular levels of the moment always exist. The modes concentrate on the level sets of the moment map, and in fact they concentrate on the hyperbolic closed geodesic.

5.1. Mass concentration of special eigenfunctions on hyperbolic orbits in the quantum integrable case. The mass profile of scarring eigenfunctions near a hyperbolic in the completely integrable case is studied in $[\mathbf{C V P}]$ on tubes of fixed radius and in $[\mathbf{N V}, \mathbf{T Z 2}]$ on tubes of shrinking radius. Let $\gamma \subset S^{*} M$ be a closed hyperbolic geodesic of an $(M, g)$ with completely integrable geodesic flow and for which $\Delta_{g}$ is quantum integrable (i.e. commutes with a maximal set of pseudo-differential operators; see [TZ2] for background). We then consider joint eigenfunctions $\Delta_{g}$ and of these operators. It is known (see e.g. [TZ2], Lemma 6) that there exists a special sequence of eigenfunctions concentrating on the momentum level set of $\gamma$. We will call them (in these notes) the $\gamma$-sequence.

Assume for simplicity that the moment level set of $\gamma$ just consists of the orbit together with its stable/unstable manifolds. Then it is proved in [TZ2] that the mass of $\varphi_{\mu}$ in the shrinking tube of radius $h^{\delta}$ around $\gamma$ with $\delta<\frac{1}{2}$ is $\simeq(1-2 \delta)$ (see also $[\mathbf{N V}]$ for a closely related result in two dimensions). Thus, the mass profile of such scarring integrable eigenfunctions only differs by the numerical factor $(1-2 \delta)$ from the mass profile of Gaussian beams. 
The difference is that the "tails" in the hyperbolic case are longer. Also the peak is logarithmically smaller than in the elliptic case (a somewhat weaker statement is proved in $[\mathbf{T Z 2}])$.

Let us state the result precisely and briefly sketch the argument. It makes an interesting comparison to the situation discussed later on of possible scarring in the Anosov case.

We denote by $\pi: S^{*} M \rightarrow M$ the standard projection and let $\pi(\gamma)$ be the image of $\gamma$ in $M$. We denote by $T_{\epsilon}(\pi(\Lambda))$ the tube of radius $\epsilon$ around $\pi(\Lambda)$. For $0<\delta<1 / 2$, we introduce a cutoff $\chi_{1}^{\delta}(x ; \hbar) \in C_{0}^{\infty}(M)$ with $0 \leq \chi_{1}^{\delta} \leq 1$, satisfying

- (i) $\operatorname{supp} \chi_{1}^{\delta} \subset T_{\hbar^{\delta}}(\pi(\gamma))$

- (ii) $\chi_{1}^{\delta}=1$ on $T_{3 / 4 \hbar^{\delta}}(\pi(\gamma))$.

THEOREM 5.2. Let $\gamma$ be a hyperbolic closed orbit in $(M, g)$ with quantum integrable $\Delta_{g}$, and let $\left\{\varphi_{\mu}\right\}$ be an $L^{2}$ normalized $\gamma$-sequence of joint eigenfunctions Then for any $0 \leq \delta<1 / 2, \lim _{\hbar \rightarrow 0}\left(O p_{\hbar}\left(\chi_{1}^{\delta}\right) \varphi_{\mu}, \varphi_{\mu}\right) \geq(1-2 \delta)$.

5.1.1. Outline of proof. For simplicity we assume $\operatorname{dim} M=2$. Let $\chi_{2}^{\delta}(x, \xi ; \hbar) \in C_{0}^{\infty}\left(T^{*} M ;[0,1]\right)$ be a second cutoff supported in a radius $\hbar^{\delta}$ tube, $\Omega(\hbar)$, around $\gamma$ with $\Omega(\hbar) \subset$ supp $\chi_{1}^{\delta}$ and such that $\chi_{1}^{\delta}=1$ on supp $\chi_{2}^{\delta}$. Thus, $\chi_{1}^{\delta}(x, \xi) \geq \chi_{2}^{\delta}(x, \xi)$, for any $(x, \xi) \in T^{*} M$. By the Garding inequality, there exists a constant $C_{1}>0$ such that:

$$
\left(O p_{\hbar}\left(\chi_{1}^{\delta}\right) \varphi_{\mu}, \varphi_{\mu}\right) \geq\left(O p_{\hbar}\left(\chi_{2}^{\delta}\right) \varphi_{\mu}, \varphi_{\mu}\right)-C_{1} \hbar^{1-2 \delta} .
$$

We now conjugate the right side to the model setting of $S^{1} \times \mathbb{R}^{1}$, i.e. the normal bundle $N_{\gamma}$ to $\gamma$. The conjugation is done by $\hbar$ Fourier integral operators and is known as conjugation to quantum Birkhoff normal form. In the model space, the conjugate of $\Delta_{g}$ is a function of $D_{s}=\frac{\partial}{i \partial s}$ along $S^{1}$ and the dilation operator $\hat{I}^{h}:=\hbar\left(D_{y} y+y D_{y}\right)$ along $\mathbb{R}$. By Egorov's theorem

$$
\left(O p_{\hbar}\left(\chi_{2}^{\delta}\right) \varphi_{\mu}, \varphi_{\mu}\right)=|c(\hbar)|^{2}\left(O p_{\hbar}\left(\chi_{2}^{\delta} \circ \kappa\right) u_{\mu}, u_{\mu}\right)-C_{3} \hbar^{1-2 \delta}
$$

where $u_{\mu}(y, s ; \hbar)$ is the model joint eigenfunction of $D_{s}, \hat{I}^{h}$, and $c(\hbar)$ is a normalizing constant. This reduces our problem to estimating the explicit matrix elements $\left(O p_{\hbar}\left(\chi_{2}^{\delta} \circ \kappa\right) u_{\mu}, u_{\mu}\right)$ of the special eigenfunctions in the model setting. The operator $\hat{I}^{h}$ has a continuous spectrum with generalized eigenfunctions $y^{-1 / 2+i \lambda / \hbar}$. The eigenfunctions on the "singular" level $\gamma$ correspond to $\lambda \sim E \hbar$. A caluclation shows that the mass in the model setting is given by

$$
M_{h}=\frac{1}{\log \hbar}\left(\int_{0}^{\infty} \chi\left(\hbar \xi / \hbar^{\delta}\right)\left|\int_{0}^{\infty} e^{-i x} x^{-1 / 2+i \lambda / \hbar} \chi\left(x / \hbar^{\delta} \xi\right) d x\right|^{2} \frac{d \xi}{\xi}\right) .
$$

Analysis of (61) shows that the right side tends to $1-2 \delta$ as $\hbar \rightarrow 0$ if $\lambda \sim E \hbar$ (see $§ 5.3$ for a detailed discussion). 
5.2. Comparison to Anosov case. This large mass profile may be a special feature of integrable systems, reflecting the fact that the stable and unstable manifolds of the hyperbolic closed orbit coincide. A heuristic picture of the mass concentration in this case is as follows: Since the eigenfunction is a stationary state, its mass must be asymptotically invariant under the geodesic flow. Since the flow compresses things exponentially in the stable direction and expands things in the unstable direction, the mass can only concentrate on the fixed closed geodesic and on the unstable manifold $W^{u}$. But $W^{u}$ returns to $\gamma$ as the stable manifold $W^{u}$ (like a figure 8). Hence, the only invariant measure is the one supported on the closed geodesic and the the mass can only concentrate there. Although it does not seem to have been proved in detail yet, it is very plausible that the mass concentration in the integrable case provides an upper bound for any $(M, g)$, i.e. it has "extremal" mass concentration.

In the Anosov case, the stable and unstable manifolds of $\gamma$ are transverse, so the dynamical picture is completely different. First, there is no obvious mechanism as in the integrable case forcing mass of any sequence of eigenfunctions to concentrate on the Lagrangian manifold formed by $\gamma$ and $W^{u}$ in the Anosov case. If mass did concentrate around $\gamma$, it would still be forced to concentrate on $\gamma$ and on $W^{u}$, but $W^{u}$ becomes dense in $S^{*} M$. Hence some of the mass must spread out uniformly over $S^{*} M$ and is lost from a neighborhood of $\gamma$. This makes it plausible that one does not get mass concentration for eigenfunctions around hyperbolic closed orbits of Anosov systems.

Yet, in the "cat map" analogue, there do exist scarring eigenfunctions [FNB, FN] for a special sparse sequence of Planck constants. The multiplicities of the eigenvalues of the cat map for this sequence saturate the bound $\hbar^{-1} /|\log \hbar|$, and therefore one can build up eigenfunctions with very unexpected properties. There is a surprising quantum mechanism forcing concentration of special modes at hyperbolic fixed points which was discovered by Faure-Nonnenmacher-de Bièvre. The eigenfunction amplitude spreads out along $\hbar^{-1 / 2}$ segments of $W^{u}$. These segments are $\hbar^{1 / 2}$ dense and so there is interference between the amplitudes on close pieces of $W^{u}$. The interference is constructive along $W^{s}$ and the mass builds up there and then as in the integrable case gets recycled back to the hyperbolic orbit.

It is not known whether this phenomenon occurs in the Riemannian setting. It is presumably related to the existence of sparse subsequences of eigenvalues with the same large multiplicities.

5.3. Details on $M_{h}$. The proof that (61) tends to $1-2 \delta$ as $\hbar \rightarrow 0$ if $\lambda \sim E \hbar$ is rather sketchy in [TZ2]. In dimension one, the proof in a model case was given in $[\mathbf{N V}]$ (we thank S. Nonnenmacher for a clarifying discussion on this point). We supply the details in the general case here as an addendum to [TZ2], to which we refer for further background. 
To estimate $M_{h}$, we assume $\lambda \sim E \hbar$ and let $\chi \in C_{0}^{\infty}(\mathbb{R} ;[0,1])$ with $\chi(x)=1$ for $|x| \leq 1$ and $\chi(x)=0$ for $|x| \geq 2$. Note that we divide by $|\log \hbar|$ in the model distribution $u_{h}(x)=\frac{1}{\sqrt{|\log \hbar|}} x^{-1 / 2+i E / \hbar} Y(x)$, so that $\left\|O p_{\hbar}(\chi) u_{h}\right\|_{L^{2}} \sim 1$. To estimate the mass on shrinking tubes of size $\hbar^{\delta}$ we write

$$
\begin{aligned}
M_{h}= & |\log \hbar|^{-1} \int_{0}^{\infty} \chi\left(\hbar^{1-\delta} \xi\right)\left|\int_{0}^{\infty} e^{-i x} x^{-1 / 2+i \lambda / \hbar} \chi\left(x / \hbar^{\delta} \xi\right) d x\right|^{2} \frac{d \xi}{\xi} \\
= & |\log \hbar|^{-1} \int_{0}^{\infty} \chi\left(\hbar^{1-\delta} \xi\right)\left|\int_{0}^{\infty} e^{-i x} x^{-1 / 2+i \lambda / \hbar} \chi\left(x / \hbar^{\delta} \xi\right) d x\right|^{2} \frac{d \xi}{\xi} \\
= & |\log \hbar|^{-1} \int_{0}^{\hbar^{\delta-1}} \frac{d \xi}{\xi}\left|\int_{0}^{\infty} e^{-i x} x^{-1 / 2+i \lambda / \hbar} \chi\left(x / \hbar^{\delta} \xi\right) d x\right|^{2} \\
& +\mathcal{O}\left(|\log \hbar|^{-1}\right)(*)
\end{aligned}
$$

The last step follows since

$$
\begin{aligned}
& |\log \hbar|^{-1} \int_{\hbar^{\delta-1}}^{2 \hbar^{\delta-1}} \frac{d \xi}{\xi}\left|\int_{0}^{\infty} e^{-i x} x^{-1 / 2+i \lambda / \hbar} \chi\left(x / \hbar^{\delta} \xi\right) d x\right|^{2} \\
& \quad=|\log \hbar|^{-1} \int_{\hbar^{\delta-1}}^{2 \hbar^{\delta-1}} \frac{d \xi}{\xi}\left|\int_{1}^{\infty} e^{-i x} x^{-1 / 2+i \lambda / \hbar} \chi\left(x / \hbar^{\delta} \xi\right) d x+\mathcal{O}(1)\right|^{2} \\
& \quad=\mathcal{O}\left(|\log \hbar|^{-1}\right) .
\end{aligned}
$$

The last estimate follows by integration by parts, since when $\xi \geq \hbar^{\delta-1}$ we have $\hbar^{\delta} \xi \geq \hbar^{2 \delta-1}$ and we assume that $2 \delta-1<0$ so $D_{x}\left(\chi\left(x / \hbar^{\delta} \xi\right)\right)=$ $\mathcal{O}\left(\hbar^{-\delta} \xi^{-1}\right) \rightarrow 0$. Also, $|\Gamma(1 / 2+i \lambda / \hbar)|^{2}=|\Gamma(1 / 2+i E)|^{2}=\frac{\pi}{\cosh (\pi E)}=O(1)$. To simplify $(*)$ we first make a change of variables $\xi \mapsto \hbar^{\delta} \xi$ and get $M_{h}=|\log \hbar|^{-1} \int_{0}^{\hbar^{2 \delta-1}} \frac{d \eta}{\eta}\left|\int_{0}^{\infty} e^{-i x} x^{-1 / 2+i \lambda / \hbar} \chi(x / \eta) d x\right|^{2}+\mathcal{O}\left(|\log \hbar|^{-1}\right)(* *)$

Next, we get rid of the interval $0 \leq \eta \leq 1$ by observing that when $\eta \in[0,1]$,

$$
\left|\int_{0}^{\infty} e^{-i x} x^{-1 / 2+i \lambda / \hbar} \chi(x / \eta) d x\right| \leq \int_{0}^{2 \eta} x^{-1 / 2} d x=\mathcal{O}\left(\eta^{1 / 2}\right)
$$

and so,

$$
\begin{aligned}
& |\log \hbar|^{-1} \int_{0}^{1} \frac{d \eta}{\eta}\left|\int_{0}^{\infty} e^{-i x} x^{-1 / 2+i \lambda / \hbar} \chi(x / \eta) d x\right|^{2} \ll|\log \hbar|^{-1} \int_{0}^{1} \eta^{-1} \eta d \eta \\
& \quad \ll|\log \hbar|^{-1} .
\end{aligned}
$$

Thus,

$$
M_{h}=|\log \hbar|^{-1} \int_{1}^{\hbar^{2 \delta-1}} \frac{d \eta}{\eta}\left|\int_{0}^{\infty} e^{-i x} x^{-1 / 2+i \lambda / \hbar} \chi(x / \eta) d x\right|^{2}+\mathcal{O}\left(|\log \hbar|^{-1}\right) .
$$


Next, one gets rid of the cutoff $\chi(x / \eta)$ by integrating by parts. When $\eta \geq 1$,

$$
\begin{aligned}
\int_{\eta}^{\infty} e^{-i x} x^{-1 / 2+i \lambda / \hbar} \chi(x / \eta) d x & =\int_{\eta}^{\infty} D_{x}\left(e^{-i x}\right) x^{-1 / 2+i \lambda / \hbar} \chi(x / \eta) d x \\
& =\mathcal{O}\left(\eta^{-1 / 2}\right) .
\end{aligned}
$$

So the $[\eta, \infty]$-range of integration in $x$ gives a contribution to $M_{h}$ that is bounded by

$$
C|\log \hbar|^{-1} \int_{1}^{\hbar^{2 \delta-1}} \frac{d \eta}{\eta} \eta^{-1} \ll|\log \hbar|^{-1} .
$$

The end result is the formula

$$
M_{h}=|\log \hbar|^{-1} \int_{1}^{\hbar^{2 \delta-1}} \frac{d \eta}{\eta}\left|\int_{0}^{\eta} e^{-i x} x^{-1 / 2+i \lambda / \hbar} d x\right|^{2}+\mathcal{O}\left(|\log \hbar|^{-1}\right) .
$$

By contour deformation, for $\eta \geq 1$,

$$
\left|\int_{0}^{\eta} e^{-i x} x^{-1 / 2+i \lambda / \hbar} d x\right|^{2}=\left|\Gamma(1 / 2+i \lambda / \hbar)+\mathcal{O}\left(\eta^{-1 / 2}\right)\right|^{2}(* * *)
$$

Substitution into (64) gives

$$
M_{h}=|\log \hbar|^{-1}|\Gamma(1 / 2+i \lambda / \hbar)|^{2} \int_{1}^{\hbar^{2 \delta-1}} \frac{d \eta}{\eta}+\mathcal{O}\left(|\log \hbar|^{-1}\right) .
$$

This last step follows since the $\mathcal{O}\left(\eta^{-1 / 2}\right)$-terms in $(* * *)$ give a contribution to $M_{\hbar}$ that is $\ll|\log \hbar|^{-1} \int_{1}^{\hbar^{2 \delta-1}} \frac{d \eta}{\eta} \eta^{-1 / 2}=\mathcal{O}\left(|\log \hbar|^{-1}\right)$.

The final formula follows immediately from (65). With $\lambda \sim E \hbar$, one gets

$$
M_{h} \sim|\Gamma(1 / 2+i E)|^{2}(1-2 \delta)
$$

Since $|\Gamma(1 / 2+i E)|^{2}=\frac{\pi}{\cosh \pi E}$, which is bounded away from zero, it follows that (61) tends to $1-2 \delta$ as $\hbar \rightarrow 0$

\section{Boundary quantum ergodicity and quantum ergodic restriction}

In this section, we briefly discuss quantum ergodic restriction theorems. The general question is whether restrictions of quantum ergodic eigenfunctions to hypersurfaces (or microlocally, to cross-sections of the geodesic flow) are quantum ergodic on the hypersurfaces. We only consider here the case where the hypersurface is the boundary of a domain with boundary, which was studied in $[\mathbf{G L}, \mathbf{H Z}, \mathbf{B u}]$. More general quantum ergodic restriction theorems are given in $[\mathbf{T Z 3}]$. The boundary results play an important role in the recent scarring results of A. Hassell for eigenfunctions on the stadium. 
We thus consider the boundary values $u_{j}^{b}$ of interior eigenfunctions

$$
\left\{\begin{array}{l}
\Delta u_{j}=\lambda_{j}^{2} u_{j} \text { in } \Omega, \quad\left\langle u_{j}, u_{k}\right\rangle_{L^{2}(\Omega)}=\delta_{j k}, \\
\left.B u_{j}\right|_{Y}=0, \quad Y=\partial \Omega
\end{array}\right.
$$

of the Euclidean Laplacian $\Delta$ on a compact piecewise smooth domain $\Omega \subset$ $\mathbb{R}^{n}$ and with classically ergodic billiard map $\beta: B^{*} Y \rightarrow B^{*} Y$, where $Y=$ $\partial \Omega$. Here $A_{h}$ is a zeroth order semiclassical pseudodifferential operator on $Y$. The relevant notion of boundary values (i.e. Cauchy data) $u_{j}^{b}$ depends on the boundary condition. We only consider the boundary conditions

$$
B u= \begin{cases}\left.u\right|_{Y}, & \text { Dirichlet } \\ \partial_{\nu} u \mid Y, & \text { Neumann }\end{cases}
$$

Let $\Delta_{B}$ denote the positive Laplacian on $\Omega$ with boundary conditions $B u=$ 0 . Then $\Delta_{B}$ has discrete spectrum $0<\lambda_{1}<\lambda_{2} \leq \cdots \rightarrow \infty$, where we repeat each eigenvalue according to its multiplicity, and for each $\lambda_{j}$ we may choose an $L^{2}$ normalized eigenfunction $u_{j}$.

The algebra of observables in the boundary setting is the algebra $\Psi_{h}^{0}(Y)$ of zeroth order semiclassical pseudodifferential operators on $Y$, depending on the parameter $h \in\left[0, h_{0}\right]$. We denote the symbol of $A=A_{h} \in \Psi_{h}^{0}(Y)$ by $a=a(y, \eta, h)$. Thus $a(y, \eta)=a(y, \eta, 0)$ is a smooth function on $T^{*} Y$.

To each boundary condition $B$ corresponds

- A specific notion of boundary value $u_{j}^{b}$ of the eigenfunctions $u_{j}$. We denote the $L^{2}$-normalized boundary values by $\hat{u}_{j}^{b}=u_{j}^{b} /\left\|u_{j}^{b}\right\|$.

- A specific measure $d \mu_{B}$ on $B^{*}(Y)$.

- A specific state $\omega_{B}$ on the space $\Psi_{h}^{0}(Y)$ of semiclassical pseuodifferential operators of order zero defined by

$$
\omega_{B}(A)=\frac{4}{\operatorname{vol}\left(S^{n-1}\right) \operatorname{vol}(\Omega)} \int_{B^{*} Y} a(y, \eta) d \mu_{B} .
$$

Here is a table of the relevant boundary value notions. In the table, $d \sigma$ is the natural symplectic volume measure on $B^{*} Y$. We also define the function $\gamma(q)$ on $B^{*} Y$ by

$$
\gamma(q)=\sqrt{1-|\eta|^{2}}, \quad q=(y, \eta)
$$

\begin{tabular}{|c|c|c|c|}
\hline \multicolumn{4}{|c|}{ Boundary Values } \\
\hline B & $B u$ & $u^{b}$ & $d \mu_{B}$ \\
\hline Dirichlet & $\left.u\right|_{Y}$ & $\left.\lambda^{-1} \partial_{\nu} u\right|_{Y}$ & $\gamma(q) d \sigma$ \\
\hline Neumann & $\left.\partial_{\nu} u\right|_{Y}$ & $\left.u\right|_{Y}$ & $\gamma(q)^{-1} d \sigma$ \\
\hline
\end{tabular}

The limit states are determined by dictated by the local Weyl law for the boundary condition $B$. 
Lemma 6.1. Let $A_{h}$ be either the identity operator on $Y$ or a zeroth order semiclassical operator on $Y$ with kernel supported away from the singular set. Then for any of the above boundary conditions $B$, we have:

$$
\begin{aligned}
& \lim _{\lambda \rightarrow \infty} \frac{1}{N(\lambda)} \sum_{\lambda_{j} \leq \lambda}\left\langle A_{h_{j}} u_{j}^{b}, u_{j}^{b}\right\rangle=\omega_{B}(A), \quad B=\text { Neumann, } \\
& \lim _{\lambda \rightarrow \infty} \frac{1}{N(\lambda)} \sum_{\lambda_{j} \leq \lambda}\left\langle A_{h_{j}} u_{j}^{b}, u_{j}^{b}\right\rangle=\omega_{B}(A), \quad B=\text { Dirichlet. }
\end{aligned}
$$

The boundary quantum ergodic theorem is:

Theorem 6.2. [HZ, GL, Bu] Let $\Omega \subset \mathbb{R}^{n}$ be a bounded piecewise smooth manifold with ergodic billiard map. Let $\left\{u_{j}^{b}\right\}$ be the boundary values of the eigenfunctions $\left\{u_{j}\right\}$ of $\Delta_{B}$ on $L^{2}(\Omega)$ in the sense of the table above. Let $A_{h}$ be a semiclassical operator of order zero on $Y$. Then there is a subset $S$ of the positive integers, of density one, such that

$$
\begin{aligned}
& \lim _{j \rightarrow \infty, j \in S}\left\langle A_{h_{j}} u_{j}^{b}, u_{j}^{b}\right\rangle=\omega_{B}(A), \quad B=\text { Neumann }, \\
& \lim _{j \rightarrow \infty, j \in S}\left\langle A_{h_{j}} u_{j}^{b}, u_{j}^{b}\right\rangle=\omega_{B}(A), \quad B=\text { Dirichlet },
\end{aligned}
$$

where $h_{j}=\lambda_{j}^{-1}$ and $\omega_{B}$ is as in (68).

In the case $A=I$ and for the Neumann boundary condition, we have

$$
\lim _{j \rightarrow \infty, j \in S}\left\|u_{j}^{b}\right\|_{L^{2}(Y)}^{2}=\frac{2 \operatorname{vol}(Y)}{\operatorname{vol}(\Omega)}
$$

while for the Dirichlet boundary condition,

$$
\lim _{j \rightarrow \infty, j \in S}\left\|u_{j}^{b}\right\|_{L^{2}(Y)}^{2}=\frac{2 \operatorname{vol}(Y)}{n \operatorname{vol}(\Omega)} .
$$

6.1. Sketch of proof. The fact that the quantum limit state $\omega_{B}$ and the corresponding measure $d \mu_{B}$ are not the natural symplectic volume measure $d \sigma$ on $B^{*} Y$ is due to the fact that the quantum dynamics is defined by an endomorphism rather than an automorphism of the observable algebra. In the Neumann case, the dynamics are generated by the operator $F_{h}$ on $Y$ with kernel

$$
\begin{gathered}
F_{h}\left(y, y^{\prime}\right)=2 \frac{\partial}{\partial \nu_{y}} G_{0}\left(y, y^{\prime}, h^{-1}\right), \quad y \neq y^{\prime} \in Y, \text { where } \\
G_{0}\left(y, y^{\prime}, \lambda\right)=\frac{i}{4} \lambda^{d-2}\left(2 \pi \lambda\left|z-z^{\prime}\right|\right)^{-(d-2) / 2} \mathrm{Ha}_{d / 2-1}^{(1)}\left(\lambda\left|z-z^{\prime}\right|\right)
\end{gathered}
$$

is the free outgoing Green function on $\mathbb{R}^{d}$. These are (almost) semi-classical Fourier integral operators whose phase functions, the boundary distance function $d_{b}\left(y, y^{\prime}\right)=\mid y-y^{\prime}\left(y, y^{\prime} \in Y\right)$ generates the billiard map. It is well 
known that this operator leaves the boundary values of Neumann eigenfunctions $u_{j}^{b}$ invariant:

$$
F_{h_{j}} u_{j}^{b}=u_{j}^{b}, \quad j=1,2, \ldots
$$

It follows that the states

$$
\rho_{j}(A):=\left\langle A_{h_{j}} u_{j}^{b}, u_{j}^{b}\right\rangle
$$

are invariant for $F_{h_{j}}$. The family $\left\{F_{h}\right\}$ defines a semiclassical Fourier integral operator associated to the billiard map $\beta$ (for convex $\Omega$ ), plus some negligible terms. The quantum dynamics on $\Psi_{h}^{0}(Y)$ is thus generated by the conjugation

$$
\alpha_{h_{j}}\left(A_{h_{j}}\right)=F_{h_{j}}^{*} A_{h_{j}} F_{h_{j}}
$$

This is analogous to the interior dynamics generated by the wave group $U_{B}^{t}$ with the boundary conditions, but unlike $U_{B}^{t}, F_{h}$ is not unitary or even normal. Indeed, the zeroth order part of $F_{h}^{*} F_{h}$ is a semiclassical pseudodifferential operator with a non-constant symbol.

The Egorov type result for the operator $F_{h}$ is as follows: Let $\beta$ denote the billiard map on $B^{*} Y^{o}$ and let $A_{h}=\mathrm{Op}\left(a_{h}\right)$ be a zeroth order operator whose symbol $a(y, \eta, 0)$. Let $\gamma$ be given by $(69)$. Then

$$
F_{h}^{*} A_{h} F_{h}=\tilde{A}_{h}+S_{h}
$$

where $\tilde{A}_{h}$ is a zeroth order pseudodifferential operator and $\left\|S_{h}\right\|_{L^{2} \rightarrow L^{2}} \leq C h$. The symbol of $\tilde{A}_{h}$ is

$$
\tilde{a}= \begin{cases}\gamma(q) \gamma(\beta(q))^{-1} a(\beta(q)), & q \in B^{*} Y \\ 0, & q \notin B^{*} Y .\end{cases}
$$

This is a rigorous version of the statement that $F_{h}$ quantizes the billiard ball map. This Egorov theorem is relevant to the Neumann boundary problem. In the Dirichlet case, the relevant operator is $F_{h}^{*}$.

The unusual transformation law of the symbol reflects the fact that (75) is not an automorphism. In the spectral theory of dynamical systems, one studies the dynamics of the billiard map $\beta$ on $B^{*} Y$ through the associated "Koopman" operator

$$
\mathcal{U}: L^{2}\left(B^{*} Y, d \sigma\right) \rightarrow L^{2}\left(B^{*} Y, d \sigma\right), \mathcal{U} f(\zeta)=f(\beta(\zeta))
$$

Here, $d \sigma$ denotes the usual $\beta$-invariant symplectic volume measure on $B^{*} Y$. From the symplectic invariance it follows that $\mathcal{U}$ is a unitary operator. When $\beta$ is ergodic, the unique invariant $L^{2}$-normalized eigenfunction is a constant $c$.

However, Egorov's theorem (76) in the boundary reduction involves the positive function $\gamma \in C^{\infty}\left(B^{*} Y\right)$, and the relevant Koopman operator is

$$
T f(\zeta)=\frac{\gamma(\zeta)}{(\gamma(\beta(\zeta)))} f(\beta(\zeta)) .
$$


Then $T$ is not unitary on $L^{2}\left(B^{*} Y, d \sigma\right)$. Instead one has:

- (i) The unique positive $T$-invariant $L^{1}$ function is given by $\gamma$.

- (ii) $T$ is unitary relative to the inner product $\langle\langle\rangle$,$\rangle on B^{*} Y$ defined by the measure $d \nu=\gamma^{-2} d \sigma$.

- (iii) When $\beta$ is ergodic, the orthogonal projection $P$ onto the invariant $L^{2}$-eigenvectors has the form

$$
P(f)=\frac{\langle\langle f, \gamma\rangle\rangle}{\langle\langle\gamma, \gamma\rangle\rangle} \gamma=\frac{1}{\operatorname{vol}\left(B^{*} Y\right)}\left[\int_{B^{*} Y} f \gamma^{-1} d \sigma\right] \gamma=c \omega_{\mathrm{Neu}}(f) \gamma
$$

where $c$ is as in (117).

The proof of Theorem 6.2 then runs along similar lines to that of Theorem 4.1 but adjusted to the fact that that $T$ is not unitary.

\section{Hassell's scarring result for stadia}

This section is an exposition of Hassell's scarring result for the Bunimovich stadium. We follow $[\mathbf{H a s}]$ and $[\mathbf{Z 7}]$.

A stadium is a domain $X=R \cup W \subset \mathbb{R}^{2}$ which is formed by a rectangle $R=[-\alpha, \alpha]_{x} \times[-\beta, \beta]_{y}$ and where $W=W_{-\beta} \cup W_{\beta}$ are half-discs of radius $\beta$ attached at either end. We fix the height $\beta=\pi / 2$ and let $\alpha=t \beta$ with $t \in[1,2]$. The resulting stadium is denoted $X_{t}$.

It has long been suspected that there exist exceptional sequences of eigenfunctions of $X$ which have a singular concentration on the set of "bouncing ball" orbits of $R$. These are the vertical orbits in the central rectangle that repeatedly bounce orthogonally against the flat part of the boundary. The unit tangent vectors to the orbits define an invariant Lagrangian submanifold with boundary $\Lambda \subset S^{*} X$. It is easy to construct approximate eigenfunctions which concentrate microlocally on this Lagrangian submanifold. Namely, let $\chi(x)$ be a smooth cutoff supported in the central rectangle and form $v_{n}=\chi(x) \sin n y$. Then for any pseudo-differential operator $A$ properly supported in $X$,

$$
\left\langle A v_{n}, v_{n}\right\rangle \rightarrow \int_{\Lambda} \sigma_{A} \chi d \nu
$$

where $d \nu$ is the unique normalized invariant measure on $\Lambda$.

Numerical studies suggested that there also existed genuine eigenfunctions with the same limit. Recently, A. Hassell has proved this to be correct for almost all stadia.

TheOREM 7.1. The Laplacian on $X_{t}$ is not QUE for almost every $t \in$ $[1,2]$.

We now sketch the proof and develop related ideas on quantum unique ergodicty. The main idea is that the existence of the scarring bouncing ball quasi-modes implies that either

- There exist actual modes with a similar scarring property, or 
- The spectrum has exceptional clustering around the bouncing ball quasi-eigenvalues $n^{2}$.

Hassell then proves that the second alternative cannot occur for most stadia. We now explain the ideas in more detail.

We first recall that a quasi-mode $\left\{\psi_{k}\right\}$ is a sequence of $L^{2}$-normalized functions which solve

$$
\left\|\left(\Delta-\mu_{k}^{2}\right) \psi_{k}\right\|_{L^{2}}=O(1)
$$

for a sequence of quasi-eigenvalues $\mu_{k}^{2}$. By the spectral theorem it follows that there must exist true eigenvalues in the interval $\left[\mu_{k}^{2}-K, \mu_{k}^{2}+K\right]$ for some $K>0$. Moreover, if $\tilde{E}_{k, K}$ denotes the spectral projection for $\Delta$ corresponding to this interval, then

$$
\left\|\tilde{E}_{k, K} \psi_{k}-\psi_{k}\right\|_{L^{2}}=O\left(K^{-1}\right)
$$

To maintain consistency with (6), i.e. with our use of frequencies $\mu_{k}$ rather than energies $\mu_{k}^{2}$, we re-phrase this in terms of the projection $E_{k, K}$ for $\sqrt{\Delta}$ in the interval $\left[\sqrt{\mu_{k}^{2}-K}, \sqrt{\mu_{k}^{2}+K}\right]$. For fixed $K$, this latter interval has width $\frac{K}{\mu_{k}}$.

Given a quasimode $\left\{\psi_{k}\right\}$, the question arises of how many true eigenfunctions it takes to build the quasi-mode up to a small error.

Definition: We say that a quasimode $\left\{\psi_{k}\right\}$ of order 0 with $\left\|\psi_{k}\right\|_{L^{2}}=1$ has $n(k)$ essential frequencies if

$$
\psi_{k}=\sum_{j=1}^{n(k)} c_{k j} \varphi_{j}+\eta_{k}, \quad\left\|\eta_{k}\right\|_{L^{2}}=o(1) .
$$

To be a quasi-mode of order zero, the frequencies $\lambda_{j}$ of the $\varphi_{j}$ must come from an interval $\left[\mu_{k}-\frac{K}{\mu_{k}}, \mu_{k}+\frac{K}{\mu_{k}}\right]$. Hence the number of essential frequencies is bounded above by the number $n(k) \leq N\left(k, \frac{K}{k}\right)$ of eigenvalues in the interval. Weyl's law for $\sqrt{\Delta}$ allows considerable clustering and only gives $N\left(k, \frac{K}{k}\right)=o(k)$ in the case where periodic orbits have measure zero. For instance, the quasi-eigenvalue might be a true eigenvalue with multiplicity saturating the Weyl bound. But a typical interval has a uniformly bounded number of $\Delta$-eigenvalues in dimension 2 or equivalently a frequency interval of with $O\left(\frac{1}{\mu_{k}}\right)$ has a uniformly bounded number of frequencies. The dichotomy above reflects the dichotomy as to whether exceptional clustering of eigenvalues occurs around the quasi-eigenvalues $n^{2}$ of $\Delta$ or whether there is a uniform bound on $N(k, \delta)$.

Proposition 7.2. If there exists a quasi-mode $\left\{\psi_{k}\right\}$ of order 0 for $\Delta$ with the properties:

- (i) $n(k) \leq C, \forall k$; 
- (ii) $\left\langle A \psi_{k}, \psi_{k}\right\rangle \rightarrow \int_{S^{*} M} \sigma_{A} d \mu$ where $d \mu \neq d \mu_{L}$.

Then $\Delta$ is not QUE.

The proof is based on the following lemma pertaining to near off-diagonal Wigner distributions. It gives an "everywhere" version of the off-diagonal part of Theorem $4.1(2)$.

Lemma 7.3. Suppose that $g^{t}$ is ergodic and $\Delta$ is QUE. Suppose that $\left\{\left(\lambda_{i_{r}}, \lambda_{j_{r}}\right), i_{r} \neq j_{r}\right\}$ is a sequence of pairs of eigenvalues of $\sqrt{\Delta}$ such that $\lambda_{i_{r}}-\lambda_{j_{r}} \rightarrow 0$ as $r \rightarrow \infty$. Then $d W_{i_{r}, j_{r}} \rightarrow 0$.

Proof. Let $\left\{\lambda_{i}, \lambda_{j}\right\}$ be any sequence of pairs with the gap $\lambda_{i}-\lambda_{j} \rightarrow 0$. Then by Egorov's theorem, any weak* limit $d \nu$ of the sequence $\left\{d W_{i, j}\right\}$ is a measure invariant under the geodesic flow. The weak limit is defined by the property that

$$
\left\langle A^{*} A \varphi_{i}, \varphi_{j}\right\rangle \rightarrow \int_{S^{*} M}\left|\sigma_{A}\right|^{2} d \nu
$$

If the eigenfunctions are real, then $d \nu$ is a real (signed) measure.

We now observe that any such weak* limit must be a constant multiple of Liouville measure $d \mu_{L}$. Indeed, we first have:

$$
\left|\left\langle A^{*} A \varphi_{i}, \varphi_{j}\right\rangle\right| \leq\left|\left\langle A^{*} A \varphi_{i}, \varphi_{i}\right\rangle\right|^{1 / 2}\left|\left\langle A^{*} A \varphi_{j}, \varphi_{j}\right\rangle\right|^{1 / 2} .
$$

Taking the limit along the sequence of pairs, we obtain

$$
\left.\left.\left|\int_{S^{*} M}\right| \sigma_{A}\right|^{2} d \nu\left|\leq \int_{S^{*} M}\right| \sigma_{A}\right|^{2} d \mu_{L} .
$$

It follows that $d \nu<<d \mu_{L}$ (absolutely continuous). But $d \mu_{L}$ is an ergodic measure, so if $d \nu=f d \mu_{L}$ is an invariant measure with $f \in L^{1}\left(d \mu_{L}\right)$, then $f$ is constant. Thus,

$$
d \nu=C d \mu_{L}, \quad \text { for some constant } C .
$$

We now observe that $C=0$ if $\varphi_{i} \perp \varphi_{j}$ (i.e. if $i \neq j$ ). This follows if we substitute $A=I$ in (78), use orthogonality and (81).

We now complete the proof of the Proposition by arguing by contradiction. The frequencies must come from a shrinking frequency interval, so the hypothesis of the Proposition is satisfied. If $\Delta$ were QUE, we would have (in the notation of (77):

$$
\begin{aligned}
\left\langle A \psi_{k}, \psi_{k}\right\rangle & =\sum_{i, j=1}^{n(k)} c_{k j} c_{k i}\left\langle A \varphi_{i}, \varphi_{j}\right\rangle+o(1) \\
& =\sum_{j=1}^{n(k)} c_{k j}^{2}\left\langle A \varphi_{j}, \varphi_{j}\right\rangle+\sum_{i \neq j=1}^{n(k)} c_{k j} c_{k i}\left\langle A \varphi_{i}, \varphi_{j}\right\rangle+o(1) \\
& =\int_{S^{*} M} \sigma_{A} d \mu_{L}+o(1),
\end{aligned}
$$

by Proposition 7.3. This contradicts (ii). In the last line we used $\sum_{j=1}^{n(k)}\left|c_{k j}\right|^{2}$ $=1+o(1)$, since $\left\|\psi_{k}\right\|_{L^{2}}=1$.

QED 
7.1. Proof of Hassell's scarring result. We apply and develop this reasoning in the case of the stadium. The quasi-eigenvalues of the Bunimovich stadium corresponding to bouncing ball quasi-modes are $n^{2}$ independently of the diameter $t$ of the inner rectangle.

By the above, it suffices to show that that there exists a sequence $n_{j} \rightarrow$ $\infty$ and a constant $M$ (independent of $j$ ) so that there exist $\leq M$ eigenvalues of $\Delta$ in $\left[n_{j}^{2}-K, n_{j}^{2}+K\right]$. An somewhat different argument is given in [Has] in this case: For each $n_{j}$ there exists a normalized eigenfunction $u_{k_{j}}$ so that $\left\langle u_{k_{j}}, v_{k_{j}}\right\rangle \geq \sqrt{\frac{3}{4} M}$. It suffices to choose the eigenfunction with eigenvalue in the interval with the largest component in the direction of $v_{k_{j}}$. There exists one since

$$
\left\|\tilde{E}_{\left[n^{2}-K, n^{2}+K\right.} v_{n}\right\| \geq \frac{3}{4}
$$

The sequence $\left\{u_{n_{k}}\right\}$ cannot be Liouville distributed. Indeed, for any $\epsilon>0$, let $A$ be a self-adjoint semi-classical pseudo-differential operator properly supported in the rectangle so that $\sigma_{A} \leq 1$ and so that $\left\|(I d-A) v_{n}\right\| \leq \epsilon$ for large enough $n$. Then

$$
\begin{aligned}
\left\langle A^{2} u_{k_{j}}, u_{k_{j}}\right\rangle & =|| A u_{k_{j}} \|^{2} \geq\left|\left\langle A u_{k_{j}}, v_{k_{j}}\right\rangle\right|^{2} \\
& =\left|\left\langle u_{k_{j}}, A v_{k_{j}}\right\rangle\right|^{2} \geq\left(\left|\left\langle u_{k_{j}}, v_{k_{j}}\right\rangle\right|-\epsilon\right)^{2} \geq\left(\sqrt{\frac{3}{4} M}-\epsilon\right)^{2} .
\end{aligned}
$$

Choose a sequence of operators $A$ such that $\left\|(I d-A) v_{n}\right\| \rightarrow 0$ and so that the support of $\sigma_{A}$ shrinks to the set of bouncing ball covectors. Then the mass of any quantum limit of $\left\{u_{n_{k}}\right\}$ must have mass $\geq \frac{3}{4} M$ on $\Lambda$.

Thus, the main point is to eliminate the possibility of exceptional clustering of eigenvalues around the quasi-eigenvalues. In fact, no reason is known why no exceptional clustering should occur. Hassell's idea is that it can however only occur for a measure zero set of diameters of the inner rectangle. The proof is based on Hadamard's variational formula for the variation of Dirichlet or Neumann eigenvalues under a variation of a domain. In the case at hand, the stadium is varied by horizontally (but not vertically) expanding the inner rectangle. In the simplest case of Dirichlet boundary conditions, the eigenvalues are forced to decrease as the rectangle is expanded. The QUE hypothesis forces them to decrease at a uniform rate. But then they can only rarely cluster at the fixed quasi-eigenvalues $n^{2}$. If this ever happened, the cluster would move left of $n^{2}$ and there would not be time for a new cluster to arrive.

Here is a more detailed sketch. Under the variation of $X_{t}$ with infinitesimal variation vector field $\rho_{t}$, Hadamard's variational formula gives,

$$
\frac{d E_{j}(t)}{d t}=\int_{\partial X_{t}} \rho_{t}(s)\left(\partial_{n} u_{j}(t)(s)\right)^{2} d s .
$$


Then

$$
E_{j}^{-1} \frac{d}{d t} E_{j}(t)=-\int_{\partial X_{t}} \rho_{t}(s) u_{j}^{b}(s)^{2} d s .
$$

Let $A(t)$ be the area of $S_{t}$. By Weyl's law, $E_{j}(t) \sim c \frac{j}{A(t)}$. Since the area of $X_{t}$ grows linearly, we have on average $\dot{E}_{j} \sim-C \frac{E_{j}}{A(t)}$. Theorem 6.2 gives the asymptotics individually for almost all eigenvalues. Let

$$
f_{j}(t)=\int_{\partial X_{t}} \rho_{t}(s)\left|u_{j}^{b}(t ; s)\right|^{2} d s .
$$

Then $\dot{E}_{j}=-E_{j} f_{j}$. Then Theorem 6.2 implies that $\left|u_{j}^{b}\right|^{2} \rightarrow \frac{1}{A(t)}$ weakly on the boundary along a subsequence of density one. QUE is the hypothesis that this occurs for the entire sequence, i.e.

$$
f_{j}(t) \rightarrow \frac{k}{A(t)}>0, \quad k:=\int_{\partial S_{t}} \rho_{t}(s) d s .
$$

Hence,

$$
\frac{\dot{E}_{j}}{E}=-k A(t)(1+o(1)), \quad j \rightarrow \infty .
$$

Hence there is a lower bound to the velocity with which eigenvalues decrease as $A(t)$ increases. Eigenvalues can therefore not concentrate in the fixed quasi-mode intervals $\left[n^{2}-K, n^{2}+K\right]$ for all $t$. But then there are only a bounded number of eigenvalues in this interval; so Proposition 7.2 implies QUE for the other $X_{t}$. A more detailed analysis shows that QUE holds for almost all $t$.

\section{Matrix elements of Fourier integral operators}

Our main focus in this survey goal is on the limits of diagonal matrix elements (12) of pseudodifferential operators $A$ or order zero relative to an orthonormal basis $\left\{\varphi_{j}\right\}$ of eigenfunctions. Difficult as it is, the limiting behavior of such matrix elements is one of the more accessible properties of eigenfunctions. To obtain more information, it would be useful to expand the class of operators $A$ for which one can study matrix elements. In $\S 5$, we were essentially expanding the class from classical polyhomogeneous pseudodifferential operators to those in the "small-scale" calculus, i.e. supported in $h^{\delta}$ tubes around closed geodesics. Another way to expand the class of $A$ is to consider matrix elements of Fourier integral operators. Motivation to consider matrix elements of Fourier integral operators comes partly from the fact that Hecke operators are Fourier integral operators, and this section is a preparation for the next section on Hecke operators $\S 9$. Other examples arise in quantum ergodic restriction theorems (see [TZ3]). Details on the claims to follow will be given in a forthcoming paper. 
By an FIO (Fourier integral operator) we mean an operator whose Schwartz kernel of $F$ may be locally represented as a finite sum of oscillatory integrals,

$$
K_{F}(x, y) \sim \int_{\mathbb{R}^{N}} e^{i \varphi(x, y, \theta)} a(x, y, \theta) d \theta
$$

for some homogeneous phase $\varphi$ and amplitude $a$. The only example we have seen so far in this survey is the wave group $U^{t}$ and of course pseudodifferential operators.

The phase is said to generate the canonical relation

$$
C=\left\{\left(x, \varphi_{x}^{\prime}, y,-\varphi_{y}^{\prime}\right): \varphi_{\xi}^{\prime}(x, y, \theta)=0\right\} \subset T^{*} M \times T^{*} M .
$$

The oscillatory integral also determines the principal $\sigma_{F}$ of $F$, a $1 / 2$ density along $C$. The data $\left(C, \sigma_{F}\right)$ determines $F$ up to a compact operator. In the case of the wave group, $C$ is the graph of $g^{t}$ and the symbol is the canonical volume half-density on the graph. In the case of $\psi \mathrm{DO}$ 's, the canonical relation is the diagonal (i.e. the graph of the identity map) and the symbol is the one defined above. We denote by $I^{0}(M, \times M, C)$ the class of Fourier integral operators of order zero and canonical relation $C$.

Hecke operators are also FIO's of a simple kind. As discussed in the next section, in that case $C$ is a local canonical graph, i.e. the projections

$$
\pi_{X}: C \rightarrow T^{*} M, \quad \pi_{Y}: C \rightarrow T^{*} M
$$

are branched covering maps. Equivalently, $C$ is the graph of a finitely many to one correspondence $\chi: T^{*} M \rightarrow T^{*} M$. Simpler examples of the same type include (i) $F=T_{g}$ is translation by an isometry of a Riemannian manifold $(M, g)$ possessing an isometry, or (ii) $T f(x)=\sum_{j=1}^{k} f\left(g_{j} x\right)+f\left(g_{j}^{-1} x\right)$ on $S^{n}$ corresponding to a finite set $\left\{g_{1}, \ldots, g_{k}\right\}$ of isometries. The latter has been studied by Lubotzky-Phillips-Sarnak.

8.1. Matrix elements as linear functionals. The matrix elements

$$
\rho_{j}(F):=\left\langle F \varphi_{j}, \varphi_{j}\right\rangle
$$

define continuous linear functionals

$$
\rho_{j}: I^{0}(M \times M, C) \rightarrow \mathbb{C}
$$

with respect to the operator norm. It is simple to see that any limit $\rho_{\infty}$ of the sequence of functionals $\rho_{j}$ are functionals only of the principal symbol data $\left(C, \sigma_{F}\right)$ which is bounded by the supremum of $\sigma_{F}$. Thus, as in the pseudo-differential case, $\rho_{\infty}$ is a measure on the space $\Omega_{C}^{1 / 2}$ of continuous half-densities on $C$.

The limiting behavior of $\rho_{j}(F)$ depends greatly on whether $[F, \sqrt{\Delta}]=0$ (or is of negative order) or whether it has the same order as $F$. In effect, this is the issue of whether the canonical relation $C$ underlying $F$ is invariant under the geodesic flow or not. For instance, if $C$ is the graph of a canonical 
transformation $\chi$ and if $\chi$ moves the energy surface $S_{g}^{*} M=\left\{(x, \xi):|\xi|_{g}=1\right\}$ to a new surface $\chi\left(S_{g}^{*} M\right)$ disjoint from $S_{g}^{*} M$ then $\left\langle F \varphi_{j}, \varphi_{j}\right\rangle \rightarrow 0$. On the other hand, $\left\langle A e^{i t \sqrt{\Delta}} \varphi_{j}, \varphi_{j}\right\rangle=e^{i t \lambda_{j}}\left\langle A \varphi_{j}, \varphi_{j}\right\rangle$ so one gets the same quantum limits for $F=A e^{i t \sqrt{\Delta}}$ as for $A$ but must pick sparser subsequences to get the limit.

Hecke operators have the property that $[F, \Delta]=0$. This implies that the canonical relation $C$ is invariant under $g^{t}$. In general this is the only condition we need to study weak limits. The eigenfunction linear functionals $\rho_{k}$ are invariant in the the sense that $\rho_{k}\left(U^{-t} F U^{t}\right)=\rho_{k}(F)$.

Proposition 8.1. Let $C \subset T^{*} M \times T^{*} M$ be a local canonical graph, equipped with the pull-back of the symplectic volume measure on $T^{*} M$. Assume $C$ is invariant under $g^{t}$. Let $F \in I^{0}(M \times M, C)$ and identify its symbol with a scalar function relative to the graph half-density. Let $\rho_{\infty}$ be a weak* limit of the functionals $\rho_{j}$ on $I^{0}(M \times N, C)$. Then there exists a complex measure $\nu$ on $S C=C \cap S^{*} M \times S^{*} M$ of mass $\leq 1$ such that

$$
\rho_{\infty}(F)=\int_{S C} \sigma_{F} d \nu
$$

which satisfies $g_{*}^{t} d \nu=d \nu$.

The proof is similar to that in the $\psi \mathrm{DO}$ case.

We note that $I^{0}(M \times M, C)$ is a right and left module over $\Psi^{0}(M)$. Given $F \in I^{0}(M \times M, C)$ we consider the operators $A F, F A \in I^{0}(M \times M, C)$ where $A \in \Psi^{0}(X)$. We obtain a useful improvement on Proposition 8.1.

Proposition 8.2. With the same hypotheses as in Proposition 8.1. Assume further that $F$ is self-adjoint and that $\rho_{j}(A F) \sim \rho_{j}(F A)$. Then we have:

$$
\rho_{\infty}\left(\pi_{X}^{*} a \sigma\right)=\rho_{\infty}\left(\pi_{Y}^{*} a \sigma\right)
$$

This holds since

$\rho_{\infty}\left(\pi_{X}^{*} a \sigma\right) \sim \rho_{j}(A F)=\left\langle A F \varphi_{j}, \varphi_{j}\right\rangle=\overline{\left\langle F^{*} A^{*} \varphi_{j}, \varphi_{j}\right\rangle}=\overline{\left\langle F A^{*} \varphi_{j}, \varphi_{j}\right\rangle} \sim \pi_{Y}^{*} a \sigma$ since $\sigma_{A^{*}} \sim \overline{\sigma_{A}}$ and since $\overline{\sigma_{F}}=\sigma_{F}$.

Let us consider some simple examples. First, suppose that $F=T_{g}+T_{g}^{*}$ where $g$ is an isometry. Then $C$ is the union of the graph of the lift of $g$ to $T^{*} M$ (by its derivative) and the inverse graph. The above Proposition then says that $g_{*} \nu=\nu$. This is obvious by Egorov's theorem, and we can regard the Proposition as a generalization of Egorov's theorem to symplectic correspondences. As a second example, let $F=U^{t}$. Then $\left\langle A F \varphi_{j}, \varphi_{j}\right\rangle=$ $e^{i t \lambda_{j}}\left\langle A \varphi_{j}, \varphi_{j}\right\rangle$, so we see that sequences with unique quantum limits are sparser than in the pseudo-differential case. 


\section{QUE of Hecke eigenfunctions}

Our next topic is Hecke eigenfunctions. A detailed treatment would have to involve adelic dynamics, higher rank measure rigidity in the presence of positive entropy [LIND, LIND2] and L-functions [HS, Sound1]. Both areas are outside the scope of this survey. Fortunately, Lindenstrauss has written an expository article on adelic dynamics and higher rank rigidity for an earlier Current Developments in Mathematics [LIND2], and Soundararajan has recently lectured on his L-function results in a 2009 Clay lecture. Sarnak has recently written exposition of the new results of Soundararajan and Holowinsky-Soundararajan results is [Sar3].

So we continue in the same vein of explaining the microlocal (phase space) features of the Hecke eigenvalue problem. The distinguishing feature of Hecke eigenfunctions is that they have a special type of symmetry. As a result, their quantum limit measures have a special type of symmetry additional to geodesic flow invariance. The analysis of these additional symmetries is a key input into Lindenstrauss' QUE result. The main purpose of this section is to derive the exact symmetry. In fact, it appears that the exact symmetry was not previously determined; only a quasi-invariance condition of $[\mathbf{R S}]$ has been employed.

We begin by recalling the definition of a Hecke operator. Let $\Gamma$ be a co-compact or cofinite discrete subgroup of $G=P S L(2, \mathbb{R})$ and let $\mathbf{X}_{\Gamma}$ be the corresponding compact (or finite area) hyperbolic surface. An element $g \in G, g \notin \Gamma$ is said to be in the commensurator $\operatorname{Comm}(\Gamma)$ if

$$
\Gamma^{\prime}(g):=\Gamma \cap g^{-1} \Gamma g
$$

is of finite index in $\Gamma$ and $g^{-1} \Gamma g$. More precisely,

$$
\Gamma=\bigcup_{j=1}^{d} \Gamma^{\prime}(g) \gamma_{j}, \quad \text { (disjoint) }
$$

or equivalently

$$
\Gamma g \Gamma=\bigcup_{j=1}^{d} \Gamma \alpha_{j}, \text { where } \alpha_{j}=g \gamma_{j} .
$$

It is also possible to choose $\alpha_{j}$ so that $\Gamma g \Gamma=\bigcup_{j=1}^{d} \alpha_{j} \Gamma$. We then have a diagram of finite (non-Galois) covers:

$$
\Gamma^{\prime}(g) \backslash \mathbf{H}
$$

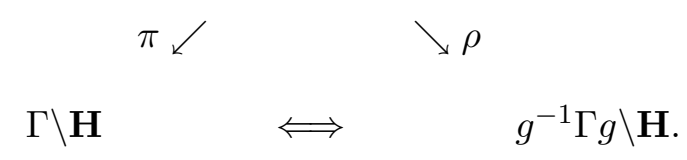

Here,

$$
\pi\left(\Gamma^{\prime}(g) z\right)=\Gamma z, \quad \rho\left(\Gamma^{\prime}(g) z\right)=g \Gamma g^{-1}\left(g \gamma_{j} z\right)=g \Gamma z
$$


where in the definition of $\rho$ any of the $\gamma_{j}$ could be used. The horizontal map is $z \rightarrow g^{-1} z$.

A Hecke operator

$$
T_{g} f(x)=\sum_{j=1}^{d} f\left(g \gamma_{j} x\right): L^{2}\left(\mathbf{X}_{\Gamma}\right) \rightarrow L^{2}\left(\mathbf{X}_{\Gamma}\right)
$$

is the Radon transform $\rho_{*} \pi^{*}$ of the diagram. We note as a result that

$$
T_{g^{-1}} u(z)=u\left(g^{-1} z\right)
$$

takes $\Gamma$-invariant functions to $g^{-1} \Gamma g$-invariant functions. The Hecke operator is thus an averaging operator over orbits of the Hecke correspondence, which is the multi-valued holomorphic map

$$
C_{g}(z)=\left\{\alpha_{1} z, \ldots, \alpha_{d} z\right\}
$$

Its graph

$$
\left.\Gamma_{g}=\left\{\left(z, \alpha_{j} z\right): z \in \mathbf{X}_{\Gamma}\right)\right\}
$$

is an algebraic curve in $\mathbf{X}_{\Gamma} \times g^{-1} X g$.

The Hecke operators $T_{g}$ form a commutative ring as $g$ varies over $\operatorname{Comm}(\Gamma)$. By a Hecke eigenfunction is meant a joint eigenfunction of $\Delta$ and the ring of Hecke operators:

$$
T_{g} u_{j}=\mu_{j}(g) u_{j}, \quad \Delta u_{j}=-\lambda_{j}^{2} u_{j} .
$$

9.1. Quantum limits on $\Gamma^{\prime}(g) \backslash \mathbf{H}$. We now considered symmetries of quantum limits of Hecke eigenfunctions. We assume $u_{j}$ is a Hecke eigenfunction. It is convenient to modify the definitions of the functionals $\rho_{k}$ as follows:

$$
\left\{\begin{array}{l}
\rho_{j}^{\prime}(A)=\frac{\left\langle A \pi^{*} u_{j}, \rho^{*} u_{j}\right\rangle}{\left\langle\pi^{*} u_{j}, \rho^{*} u_{j}\right\rangle}=\frac{\left\langle\rho_{*} A \pi^{*} u_{j}, u_{j}\right\rangle}{\left\langle\pi^{*} u_{j}, \rho^{*} u_{j}\right\rangle} \\
\sigma_{j}^{\prime}(A)=\frac{\left\langle A \pi^{*} u_{j}, \pi^{*} u_{j}\right\rangle}{\left\langle\pi^{*} u_{j}, \rho^{*} u_{j}\right\rangle} \\
\tau_{j}^{\prime}(A)=\frac{\left\langle A \pi^{*} u_{j}, \rho^{*} u_{j}\right\rangle}{\left\langle\rho^{*} u_{j}, \rho^{*} u_{j}\right\rangle}
\end{array}\right.
$$

on $A \in \Psi^{0}\left(\Gamma^{\prime}(g) \backslash \mathbf{H}\right)$. Thus, if $A_{0} \in \Psi^{0}\left(\mathbf{X}_{\Gamma}\right)$ we have,

$$
\frac{\left\langle A_{0} \rho_{*} \pi^{*} u_{j}, u_{j}\right\rangle}{\left\langle\rho_{*} \pi^{*} u_{j}, u_{j}\right\rangle}=\left\langle A_{0} u_{j}, u_{j}\right\rangle .
$$

Since Hecke operators are FIO's, limit measures of $\left\langle A T_{g} u_{j}, u_{j}\right\rangle$ and $\left\langle T_{g} A u_{j}, u_{j}\right\rangle$ live on the canonical relation underlying $T_{g}$, which is the lift 
of the graph of the Hecke correspondence to $T^{*} \mathbf{X}_{\Gamma}$. It is convenient to restate the result by forming a second diagram

$$
\Gamma_{g} \subset \mathbf{X}_{\Gamma} \times g^{-1} \Gamma g \backslash \mathbf{H}
$$

$$
\begin{array}{cl}
\pi_{1} \swarrow & \searrow \rho_{1} \\
\Gamma \backslash \mathbf{H} & \Longleftrightarrow \quad g^{-1} \Gamma g \backslash \mathbf{H} .
\end{array}
$$

Here, $\pi_{1}, \rho_{1}$ are the natural projections. In the case of arithmetic groups such as $S L(2, \mathbb{Z}), \operatorname{Comm}(\Gamma)$ is dense in $G$, i.e. there are many Hecke operators. The map

defined by

$$
\iota: \Gamma^{\prime}(g) \backslash \mathbf{H} \rightarrow \Gamma_{g}
$$

$$
\iota(z)=(\pi(z), \rho(z)),
$$

is a local diffeomorphic parametrization, and $\pi_{1} \iota=\pi, \rho_{1} \iota=\rho$.

The general Proposition 8.2 then specializes to:

Proposition 9.1. If $\omega^{\prime}$ is a weak ${ }^{*}$ limit of $\rho_{j}^{\prime}$, and if $\omega$ is the weak* limit on $\mathbf{X}_{\Gamma}$ then $\pi_{*} \omega^{\prime}=\rho_{*} \omega^{\prime}=\omega$.

We may reformulate the proposition as follows: if we lift measures and functions to the universal cover $\mathbb{H}$, then the data of the Hecke limit measures are a finite set of real signed measures $\left\{d \nu_{k}\right\}$ on the different copies of $S^{*} \mathbb{H}$. The latter must project to the former under both projections, so we get the exact invariance property,

$$
\nu=\sum_{j=1}^{d} \nu_{\alpha_{j}}=\sum_{j=1}^{d} \alpha_{j *} \nu_{\alpha_{j}},
$$

for some complex measures $\nu_{j}$ on the $j$ sheet of the cover. The sum of the masses of the $\nu_{j}$ must add up to one. This is simpler to see for Hecke operators on $S^{2}$. There we also have some number $r$ of copies of $S^{2}$ and $r$ isometries $g_{j}$ and define the Hecke operator (of Lubotzky-Phillips-Sarnak) as in (85). Then a quantum limit measure $\nu_{0}$ on $S^{*} S^{2}$ is the projection of $r$ limit measures on the copies of $S^{*} S^{2}$ under both projections.

Previously, only a quasi-invariance property of quantum limit measures of Hecke eigenfunctions was proved by Rudnick-Sarnak [RS], and used in [BL, W3] and elsewhere. In the notation above, it may be stated as

$$
\nu(E) \leq d \sum_{j}\left|\alpha_{j *} \nu_{\alpha_{j}}(E)\right| .
$$

As a simple application of (89) (which was also clear from the quasiinvariance condition), we sketch a proof that a quantum limit measure $\nu$ of a sequence of Hecke eigenfunctions for $\Gamma=S L(2, \mathbb{Z})$ cannot be a periodic orbit measure $\mu_{\gamma}$. If it were, it would have to be $\sum \nu_{j}$ as above. Each $\nu_{j}$ is an invariant signed measure so by ergodic decomposition, each must have 
the form $c_{j} \mu_{\gamma}+\tau_{j}$ where $\sum c_{j}=1$, where $\tau_{j}$ are singular with respect to $\mu_{\gamma}$ and $\sum_{j} \tau_{j}=0$. But also the images of $\nu_{j}$ under the $\alpha_{j}$ must have the same property. This is only possible if $\alpha_{j}(\gamma)=\gamma$ up to a translation by $\beta \in \Gamma$ whenever $\nu_{j} \neq 0$. At least one of the $c_{j} \neq 0$. At this point, one must actually consider the elements $\alpha_{j}$ in the Hecke operator. In the case of $P S L(2, \mathbb{Z})$ they are parabolic elements plus one elliptic element. But only a hyperbolic element can fix a geodesic (its axis).

It would be interesting to see if one can determine how the $\nu_{\alpha_{j}}$ are related to each other. Lindenstrauss's QUE result (in the next section) implies that that $\left(T_{g}\right)_{*} \nu=\nu$, which appears to say that the $\nu_{\alpha_{j}}$ are all the same. An apriori proof of this would simplify the proof of QUE.

9.2. QUE results of Lindenstrauss, Soundararajan (and Holowinsky-Soundararajan). We now briefly recall the QUE results on Hecke eigenfunctions.

Theorem 9.2. (E. Lindenstrauss [LIND]) Let $\mathbf{X}_{\Gamma}$ be a compact arithmetic hyperbolic surface. Then QUE holds for Hecke eigenfunctions, i.e. the entire sequence of Wigner distributions of Hecke eigenfunctions tends to Liouville measure.

In the non-compact finite area case of $\Gamma=P S L(2, \mathbb{Z})$, Lindenstrauss proved:

TheOREM 9.3. (E. Lindenstrauss $[\mathbf{L I N D}]$ ) Let $\mathbf{X}_{\Gamma}$ be the arithmetic hyperbolic surface defined by a congruence subgroup. Then any weak* limit of the sequence of Wigner distributions of Hecke eigenfunctions is a constant multiple of Liouville measure (the constant may depend on the sequence).

Note that the quantum ergodicity theorem does not apply in this finite area case. In the arithmetic case there is a discrete spectrum corresponding to cuspidal eigenfunctions and a continuous spectrum corresponding to Eisenstein series. The following was proved in $[\mathbf{Z 8}]$ :

TheOREM 9.4. Let $\mathbf{X}_{\Gamma}$ be the arithmetic hyperbolic surface defined by a congruence subgroup, and let $\left\{\varphi_{j}\right\}$ be any orthonormal basis of cuspidal eigenfunctions. Then for any pseudo-differential operator of order zero and with compactly supported symbol,

$$
\frac{1}{N_{c}(\lambda)} \sum_{j: \lambda_{j} \leq \lambda}\left|\left\langle A \varphi_{j}, \varphi_{j}\right\rangle-\omega(A)\right|^{2} \rightarrow 0,
$$

where $N_{c}(\lambda) \sim\left|\mathbf{X}_{\Gamma}\right| \lambda^{2}$ is the number of cuspidal eigenvalues $\leq \lambda$.

Recent work of Soundararajan that the constant equals one, i.e. there is no mass leakage at infinity. Hence the theorem is improved to

Theorem 9.5. (E. Lindenstrauss [LIND], Soundarajan [Sound1]) Let $\mathbf{X}_{\Gamma}$ be the arithmetic hyperbolic surface defined by a congruence subgroup. 
Then any weak* limit of the sequence of Wigner distributions of Hecke eigenfunctions is is normalized Liouville measure.

Holowinsky [Hol] and Holowinsky-Soundararajan [HS] have proven QUE results for holomorphic forms (i.e. for the discrete series; see §1.15.4). However, the methods are entirely different; they are based on the theory of L-functions and Poincaré series. The Hecke property is exploited through multiplicativity of Fourier coefficients.

9.3. Hecke QUE and multiplicities. A natural question (raised at the Clay talk of Soundararajan) is whether QUE holds for any orthonormal basis of eigenfunctions of an arithmetic quotient if it holds for the Hecke eigenbasis and if the discrete spectrum of $\Delta$ has bounded multiplicities. The proof that this is true is a simple modification of Proposition 7.2 and we pause to sketch it now.

Proposition 9.6. Suppose that one orthonormal basis of $\Delta$ is QUE, and that the eigenvalues have uniformly bounded multiplicities. Then all orthonormal bases are QUE.

Proof. As in Proposition 7.2, consider sequences $\left\{\left(\lambda_{i_{r}}, \lambda_{j_{r}}\right), i_{r} \neq j_{r}\right\}$ of pairs of eigenvalues of $\sqrt{\Delta}$ with $\lambda_{i_{r}}=\lambda_{j_{r}}$. We then have that $d W_{i_{r}, j_{r}} \rightarrow 0$.

This has implications for all orthonormal basis as long as eigenvalue multiplicities are uniformly bounded. We run the argument regarding modes and quasi-modes but use the QUE orthonormal basis in the role of modes and any other orthonormal basis $\left\{\psi_{k}\right\}$ in the role of quasi-modes.

Lemma 9.7. If the eigenvalue multiplicities are uniformly bounded and there exists a sequence of eigenfunctions $\left\{\psi_{k}\right\}$

- (i) $n(k) \leq C, \forall k$;

- (ii) $\left\langle A \psi_{k}, \psi_{k}\right\rangle \rightarrow \int_{S^{*} M} \sigma_{A} d \mu$ where $d \mu \neq d \mu_{L}$.

Then no orthonormal basis of eigenfunctions of $\Delta$ is QUE.

Proof. We argue by contradiction. If $\left\{\varphi_{j}\right\}$ were QUE, we would have (in the notation of (77):

$$
\begin{aligned}
\left\langle A \psi_{k}, \psi_{k}\right\rangle & =\sum_{i, j=1}^{n(k)} c_{k j} c_{k i}\left\langle A \varphi_{i}, \varphi_{j}\right\rangle+o(1) \\
& =\sum_{j=1}^{n(k)} c_{k j}^{2}\left\langle A \varphi_{j}, \varphi_{j}\right\rangle+\sum_{i \neq j=1}^{n(k)} c_{k j} c_{k i}\left\langle A \varphi_{i}, \varphi_{j}\right\rangle+o(1) \\
& =\int_{S^{*} M} \sigma_{A} d \mu_{L}+o(1),
\end{aligned}
$$

by Proposition 9.6. This contradicts (ii). In the last line we used $\sum_{j=1}^{n(k)}\left|c_{k j}\right|^{2}$ $=1+o(1)$, since $\left\|\psi_{k}\right\|_{L^{2}}=1$. 


\section{Variance estimates: Rate of quantum ergodicity and mixing}

A quantitative refinement of quantum ergodicity is to ask at what rate the sums in Theorem 4.1(1) tend to zero, i.e. to establish a rate of quantum ergodicity. More generally, we consider "variances" of matrix elements. For diagonal matrix elements, we define:

$$
V_{A}(\lambda):=\frac{1}{N(\lambda)} \sum_{j: \lambda_{j} \leq \lambda}\left|\left\langle A \varphi_{j}, \varphi_{j}\right)-\omega(A)\right|^{2} .
$$

In the off-diagonal case one may view $\left|\left\langle A \varphi_{i}, \varphi_{j}\right\rangle\right|^{2}$ as analogous to $\mid\left\langle A \varphi_{j}, \varphi_{j}\right)$ $-\left.\omega(A)\right|^{2}$. However, the sums in (57) are double sums while those of (91) are single. One may also average over the shorter intervals $[\lambda, \lambda+1]$.

10.1. Quantum chaos conjectures. It is implicitly conjectured by Feingold-Peres in $[\mathbf{F P}](11)$ that

$$
\left|\left\langle A \varphi_{i}, \varphi_{j}\right\rangle\right|^{2} \simeq \frac{C_{A}\left(\frac{\left.E_{i}-E_{j}\right)}{\hbar}\right)}{2 \pi \rho(E)},
$$

where $C_{A}(\tau)=\int_{-\infty}^{\infty} e^{-i \tau t}\left\langle V^{t} \sigma_{A}, \sigma_{A}\right\rangle d t$. In our notation, $\lambda_{j}=\hbar^{-1} E_{j}$ (with $\hbar=\lambda_{j}^{-1}$ and $E_{j}=\lambda_{j}^{2}$ ) and $\rho(E) d E \sim d N(\lambda)$.

On the basis of the analogy between $\left|\left\langle A \varphi_{i}, \varphi_{j}\right\rangle\right|^{2}$ and $\left|\left\langle A \varphi_{j}, \varphi_{j}\right)-\omega(A)\right|^{2}$, it is conjectured in $[\mathbf{F P}]$ that

$$
V_{A}(\lambda) \sim \frac{C_{A-\omega(A) I}(0)}{\lambda^{n-1} \operatorname{vol}(\Omega)} .
$$

The idea is that $\varphi_{ \pm}=\frac{1}{\sqrt{2}}\left(\varphi_{i} \pm \varphi_{j}\right)$ have the same matrix element asymptotics as eigenfunctions when $\lambda_{i}-\lambda_{j}$ is sufficiently small. But then $2\left\langle A \varphi_{+}, \varphi_{-}\right\rangle=$ $\left\langle A \varphi_{i}, \varphi_{i}\right\rangle-\left\langle A \varphi_{j}, \varphi_{j}\right\rangle$ when $A^{*}=A$. Since we are taking a difference, we may replace each matrix element by $\left\langle A \varphi_{i}, \varphi_{i}\right\rangle$ by $\left\langle A \varphi_{i}, \varphi_{i}\right\rangle-\omega(A)$ (and also for $\left.\varphi_{j}\right)$. The conjecture then assumes that $\left\langle A \varphi_{i}, \varphi_{i}\right\rangle-\omega(A)$ has the same order of magnitude as $\left\langle A \varphi_{i}, \varphi_{i}\right\rangle-\left\langle A \varphi_{j}, \varphi_{j}\right\rangle$.

10.2. Rigorous results for Hecke eigenfunctions. Let $\mathbf{X}_{\Gamma}$ be the arithmetic modular surface, with $\Gamma=S L(2, \mathbb{Z})$, and let $T_{n}, n \geqslant 1$ denote the family of Hecke operators. In addition to the Laplace eigenvalue problem (28), the Maass-Hecke eigenforms are eigenfunctions of the Hecke operators,

$$
T_{n} \varphi=\lambda_{\varphi}(n) \varphi .
$$

In the cusp, they have Fourier series expansions

$$
\varphi(z)=\sqrt{y} \sum_{n \neq 0} \rho_{\varphi}(n) K_{i r}(2 \pi|n| y) e(n x)
$$


where $K_{i r}$ is the $K$-Bessel function and $\rho_{\varphi}(n)=\lambda_{\varphi}(n) \rho_{\varphi}(1)$. A special case of the variance sums (91) is the configuration space variance sum,

$$
S_{\psi}(\lambda)=\sum_{\lambda_{j} \leq \lambda}\left|\int \psi \varphi^{2} d V\right|^{2}
$$

Following the notation of $[\mathbf{L S 2}]$ and $[\mathbf{Z h}]$,we put $d \mu_{n}=\varphi_{r_{n}}^{2} d V$.

Let $\psi \in C_{0,0}^{\infty}\left(S^{*} \mathbf{X}_{\Gamma}\right)$ and consider the distribution of

$$
\frac{1}{\sqrt{T}} \int_{0}^{T} \psi\left(g^{t}(x, \xi)\right) d t .
$$

Ratner's central limit theorem for geodesic flows implies that this random variable tends to a Gaussian with mean 0 and the variance given by the non-negative Hermitian form on $C_{0,0}^{\infty}(Y)$ :

$$
V_{\mathrm{C}}(\varphi, \psi)=\int_{-\infty}^{\infty} \int_{\Gamma \backslash S L(2, \mathbf{R})} \varphi\left(g\left(\begin{array}{cc}
e^{\frac{t}{2}} & 0 \\
0 & e^{-\frac{t}{2}}
\end{array}\right)\right) \overline{\psi(g)} d g d t .
$$

Restrict $V_{C}$ to $C_{0,0}^{\infty}(X)$. Different irreducible representations are orthogonal under the quadratic form. Maass cusp forms $\varphi$ are eigenvectors of $V_{C}$. If the Laplace eigenvalue is $\frac{1}{4}+r^{2}$, then the $V_{C}$ eigenvalue is

$$
V_{\mathrm{C}}(\varphi, \varphi)=\frac{\left|\Gamma\left(\frac{1}{4}-\frac{i r}{2}\right)\right|^{4}}{2 \pi\left|\Gamma\left(\frac{1}{2}-i r\right)\right|^{2}}
$$

In [LS, LS2], Luo and Sarnak studied the quantum variance for holomorhic Hecke eigenforms, i.e. holomorphic cusp forms in $S_{k}(\Gamma)$ of even integral weight $k$ for $\Gamma$. In this setting, the weight of the cusp form plays the role of the Laplace eigenvalue. They proved that for $\varphi, \psi \in C_{0,0}^{\infty}(X)$, as the weight $K \rightarrow \infty$,

$$
\sum_{k \leq K} \sum_{f \in H_{k}} L\left(1, \operatorname{Sym}^{2} f\right) \mu_{f}(\varphi) \overline{\mu_{f}(\psi)} \sim B(\varphi, \psi) K,
$$

where $B(\varphi, \psi)$ is a non-negative Hermitian form on $C_{0,0}^{\infty}(X)$. B is diagonalized by the orthonormal basis of Maass-Hecke cusp forms and the eigenvalues of $B$ at $\psi_{j}$ is $\frac{\pi}{2} L\left(\frac{1}{2}, \psi_{j}\right)$. For the notation $L\left(1\right.$, Sym $\left.^{2}\right)$ we refer to $[\mathbf{L S 2}, \mathbf{Z h}$.

In $[\mathbf{Z h}]$, P. Zhao generalized the result in $[\mathbf{L S}, \mathbf{L S 2}]$ to Maass cusp forms. Let $\varphi_{j}(z)$ be the $j$-th Maass-Hecke eigenform, with the Laplacian eigenvalues $\frac{1}{4}+r_{j}^{2}$.

Theorem 10.1. $[\mathbf{Z h}]$ Fix any $\epsilon>0$. Then we have

$$
\begin{aligned}
& \sum_{t_{j}}\left(e^{-\left(\frac{r_{j}-T}{T^{1-\epsilon}}\right)^{2}}+e^{-\left(\frac{r_{j}+T}{T^{1-\epsilon}}\right)^{2}}\right) L\left(1, \operatorname{sym}^{2} \varphi_{j}\right) \mu_{j}(\varphi) \overline{\mu_{j}}(\psi) \\
& \quad=T^{1-\epsilon} V(\varphi, \psi)+O\left(T^{\frac{1}{2}+\epsilon}\right),
\end{aligned}
$$


$V$ is diagonalized by the orthonormal basis $\left\{\varphi_{j}\right\}$ of Maass-Hecke cusp forms and the eigenvalue of $V$ at a Maass-Hecke cusp form $\varphi$ is

$$
L\left(\frac{1}{2}, \varphi\right) V_{\mathrm{C}}(\varphi, \varphi) .
$$

It would be desirable to remove the weights. The unweighted version should be (P. Zhao, personal communication; to appear)

$$
\sum_{\lambda_{j} \leq \lambda} \mu_{j}(\varphi) \bar{\mu}_{j}(\psi) \sim \lambda V(\varphi, \psi)
$$

10.3. General case. The only rigorous result to date which is valid on general Riemannian manifolds with hyperbolic geodesic flow is the logarithmic decay:

Theorem 10.2. [ZZ4] For any $(M, g)$ with hyperbolic geodesic flow,

$$
\frac{1}{N(\lambda)} \sum_{\lambda_{j} \leq \lambda}\left|\left(A \varphi_{j}, \varphi_{j}\right)-\omega(A)\right|^{2 p}=O\left(\frac{1}{(\log \lambda)^{p}}\right) .
$$

It is again based on Ratner's central limit theorem for the geodesic flow. The logarithm reflects the exponential blow up in time of remainder estimates for traces involving the wave group associated to hyperbolic flows and thus the necessity of keeping the time less than the Ehrenfest time (18). It would be surprising if the logarithmic decay is sharp for Laplacians. However, R. Schubert shows in [Schu, Schu2] that the estimate is sharp in the case of two-dimensional hyperbolic quantum cat maps. Hence the estimate cannot be improved by semi-classical arguments that hold in both settings.

10.4. Variance and Patterson-Sullivan distributions. When $\int_{S^{*}} \mathbf{X}_{\Gamma} a d \mu_{L}=0$, the Luo-Sarnak variance results for Hecke eigenfunctions have the form,

$$
\frac{1}{N(\lambda)} \sum_{j:\left|r_{j}\right| \leq \lambda}\left|\left\langle a, d W_{i r_{j}}\right\rangle\right|^{2} \sim \frac{1}{\lambda} V(a, a) .
$$

Even if one knew that asymptotics of this kind should occur, it does not seem apriori obvious that the bilinear form on the right side should be invariant under $g^{t}$ because $V(a, a)$ occurs in the $\frac{1}{\lambda}$ term in the variance asymptotics and only only knows that the Wigner distributions are invariant modulo terms of order $\frac{1}{\lambda_{j}}$. A possible way to see that the bilinear form should be $g^{t}$-invariant is via the Patterson-Sullivan distributions. From (51), we apparently have

$$
\frac{1}{N(\lambda)} \sum_{j:\left|r_{j}\right| \leq \lambda}\left|\left\langle a, d W_{i r_{j}}\right\rangle\right|^{2}=\frac{1}{N(\lambda)} \sum_{j:\left|r_{j}\right| \leq \lambda}\left|\left\langle a, d \hat{P} S_{i r_{j}}\right\rangle\right|^{2}+O\left(\lambda^{-2}\right) .
$$


Indeed, the difference is of the form $\frac{1}{N(\lambda)} \sum_{j:\left|r_{j}\right| \leq \lambda} \frac{2}{r_{j}} \operatorname{Re}\left\langle a, d \hat{P S_{i r_{j}}}\right\rangle$. $\overline{\left\langle L_{2} a, d \hat{\left.P S_{i r_{j}}\right\rangle}\right.}+O\left(\lambda^{-2}\right)$. The sum in the last expression also seems to be of order $O\left(\lambda^{-2}\right)$ (e.g. one can go back and express each factor of $P S$ with one of $W_{i r_{j}}$ and apply the Luo-Sarnak asymptotics again). Since $\hat{P} S_{i r_{j}}$ is $g^{t}$-invariant, it follows that $V(a, a)$ must be $g^{t}$ invariant. This argument is equally valid on any compact hyperbolic surface, but of course there is no proof of such asymptotics except in the arithmetic Hecke case. It would be interesting to draw further relations between $V(a, a)$ and $P S_{i r_{j}}$.

\section{Entropy of quantum limits on manifolds with Anosov geodesic flow}

So far, the results on quantum limits have basically used the symmetry (or invariance) properties of the limits. But generic chaotic systems have no symmetries. What is there to constrain the huge number of possible limits?

The recent results of Anantharaman $[\mathbf{A}]$, Anantharaman-Nonnenmacher $[\mathbf{A N}]$ and Rivière $[\mathbf{R i v}]$, give a very interesting answer to this question (see also $[\mathbf{A N K}])$ for $(M, g)$ with Anosov geodesic flow. They use the quantum mechanics to prove lower bounds on entropies of quantum limit measures. The lower bounds eliminate many of the possible limits, e.g. they disqualify finite sums of periodic orbit measures.

The purpose of this section is to present the results of $[\mathbf{A}]$ and $[\mathbf{A N}]$ in some detail. Both articles are based on a hyperbolic dispersive estimate (called the main estimate in $[\mathbf{A}]$ ) which, roughly speaking, measures the norm of quantized cylinder set operators in terms of $\hbar$ and the length of the cylinder. But they use the estimate in quite different ways. In $[\mathbf{A}]$, it is used in combination with an analysis of certain special covers of $S^{*} M$ by cylinder sets that are adapted to the eigenfunctions. One of the important results is an estimate on the topological entropy $h_{\text {top }}\left(\operatorname{supp}\left(\nu_{0}\right)\right)$ of the support of any quantum limit measure. In $[\mathbf{A N}]$ the key tool is the "entropic uncertainty principle". It leads to a lower bound for the Kolomogorv-Sinai entropy $h_{K S}\left(\nu_{0}\right)$ of the quantum limit.

There now exist several excellent and authoritative expositions of the KS entropy bounds and the entropic uncertainty principle $[\mathbf{A 2}$, ANK, AN2, CV3] in addition to the well written initial articles $[\mathbf{A}, \mathbf{A N}]$ (which take considerable care to give intuitive explanations of technical steps). For this reason, we emphasize the approach in $[\mathbf{A}]$. We closely follow the original references in discussing heuristic reasons for the lower bounds and outlining rigorous proofs. We also discuss earlier entropy lower bounds of BourgainLindenstrauss $[\mathbf{B L}]$ and Wolpert [W3] in the case of Hecke eigenfunctions.

Before stating the results, we review the various notions of entropy.

11.1. KS entropy. We first recall the definition of the KS entropy of an invariant probability measure $\mu$ for the geodesic flow. Roughly speaking, the entropy measures the average complexity of $\mu$-typical orbits. In the 
Kolmogorov-Sinai entropy, one starts with a partition $\mathcal{P}=\left(E_{1}, \ldots, E_{k}\right)$ of $S_{g}^{*} M$ and defines the Shannon entropy of the partition by $h_{\mathcal{P}}(\mu)=$ $\sum_{j=1}^{k} \mu\left(E_{j}\right) \log \mu\left(E_{j}\right)$. Under iterates of the time one map $g$ of the geodesic flow, one refines the partition from the sets $E_{j}$ to the cylinder sets of length $n$ :

$$
\mathcal{P}^{n}:=\left\{\mathcal{P}_{\alpha}^{n}:=E_{\alpha_{0}} \cap g^{-1} E_{\alpha_{1}} \cap \cdots \cap g^{-n+1} E_{\alpha_{n-1}}: \quad\left[\alpha_{0}, \ldots, \alpha_{n-1}\right] \in \mathbf{N}^{n}\right\} .
$$

One defines $h_{n}(\mathcal{P}, \mu)$ to be the Shannon entropy of this partition and then defines $h_{K S}(\mu, \mathcal{P})=\lim _{n \rightarrow \infty} \frac{1}{n} h_{n}(\mu, \mathcal{P})$. Then $h_{K S}(\mu)=\sup _{\mathcal{P}} h_{K S}(\mu, \mathcal{P})$.

The measure $\mu\left(E_{\alpha_{0}} \cap g^{-1} E_{\alpha_{1}} \cap \cdots \cap g^{-n+1} E_{\alpha_{n-1}}\right)$ is the probability with respect to $\mu$ that an orbit successively visits $E_{\alpha_{0}}, E_{\alpha_{1}}, \ldots$ The entropy measures the exponential decay rate of these probabilities for large times.

11.2. Symbolic coding and cylinder sets. In the $(M, g)$ setting, we fix a partition $\left\{M_{k}\right\}$ of $M$ and a corresponding partition $T^{*} M_{k}$ of $T^{*} M$. Let $P_{\alpha_{k}}$ be the characteristic function of $T^{*} M_{k}$. (Later it must to be smoothed out). Let $\Sigma=\{1, \ldots, \ell\}^{\mathbb{Z}}$ where $\ell$ is the number of elements of the partition $\mathcal{P}^{0)}$. To each tangent vector $v \in S^{*} M$, one can associated a unique element $I(v)=\left(\alpha_{k}\right) \in \Sigma$ so that $g^{n} v \in P_{\alpha_{j}}$ for all $n \in \mathbb{Z}$. This gives a symbolic coding map $I: S^{*} M \rightarrow \Sigma$. The time one map $g^{1}$ then conjugates under the coding map to the shift $\sigma\left(\left(\alpha_{j}\right)\right)=\left(\left(\alpha_{j+1}\right)\right)$ on admissible sequences, i.e. sequences in the image of the coding map. An invariant measure $\nu_{0}$ thus corresponds to a shift invariant measure $\mu_{0}$ on $\Sigma$.

A cylinder set $\left[\alpha_{0}, \ldots, \alpha_{n-1}\right] \subset \Sigma$ of length $n$ is the subset of $\Sigma$ formed by sequences with the given initial segment. The set of such cylinder sets of length $n$ is denoted $\Sigma_{n}$. Cylinder sets for $\Sigma$ are not the same as cylinder sets for $g^{1}$, which have the form $P_{\alpha_{0}} \cap g^{-1} P_{\alpha_{1}} \cap \cdots \cap g^{-n} P_{\alpha_{n}}$.

The $\mu_{0}$ measure of a $\Sigma$-cylinder set is by definition,

$$
\mu_{0}\left(\left[\alpha_{0}, \ldots, \alpha_{n}\right]\right)=\nu_{0}\left(P_{\alpha_{0}} \cap g^{-1} P_{\alpha_{1}} \cap \cdots \cap g^{-n+1} P_{\alpha_{n-1}}\right) .
$$

11.3. Bowen balls. Cylinder sets are closely related to Bowen balls, i.e. balls in the Bowen metric. For any smooth map $f: X \rightarrow X$, the Bowen metric at time $n$ is defined by

$$
d_{n}^{f}(x, y)=\max _{0 \leq i \leq n-1} d\left(f^{i} x, f^{i} y\right)
$$

Let $B_{n}^{f}(x, r)$ be the $r$ ball around $x$ in this metric.

In the continuous time case of the geodesic flow $g^{t}$, one defines the $d_{T}$ metric

$$
d_{T}\left(\rho, \rho^{\prime}\right)=\max _{t \in[0 T]} d\left(g^{t}(\rho), g^{t}\left(\rho^{\prime}\right)\right)
$$

The Bowen ball $B_{T}(\rho, r)$ at time $T$ is the $r$ - ball in this metric.

As with cylinder sets, if $\rho, \rho^{\prime}$ lie in $B_{T}(\rho, \epsilon)$ then their orbits are $\epsilon$-close for the interval $[0, T]$. This is not quite the same as running through the same elements of a partition but for large $T$ the balls and cylinders are 
rather similar. This is because the geodesic flow $g^{t}$ stretches everything by a factor of $e^{t}$ in the unstable direction, and contracts everything by $e^{-t}$ in the stable direction; it preserves distances along the geodesic flow. In variable curvature and in higher dimensions, the "cube" becomes a rectangular parallelopiped whose axes are determined by the Lyapunov exponents.

In the case of geodesic flows of compact hyperbolic manifolds of constant curvature -1 , Bowen balls $B_{T}(\rho, r)$ are roughly of radius $r e^{-T}$ in the unstable direction, and $r$ in the stable direction and geodesic flow directions. In the hyperbolic case of $G / \Gamma$ with co-compact $\Gamma$, the tube (in the notation of $\S 1.15 .1)$ is

$$
\left.\left.B_{T}(\rho, \epsilon)\right)=a((-r, r)) n_{-}(r, r)\right) n_{+}\left(\left(-e^{-T} r, e^{-T} r\right)\right) .
$$

To make the ball symmetric with respect to the stable and unstable directions is to make it symmetric with respect to time reversal. One uses the time interval $[-T / 2, T / 2]$ instead of $[0, T]$ and defines the new distance,

$$
d_{T}^{\prime}\left(\rho, \rho^{\prime}\right)=\max _{t \in-[T / 2, T / 2]} d^{\prime}\left(g^{t}(\rho), g^{t}\left(\rho^{\prime}\right)\right),
$$

We then denote the $r$ ball by $B_{T}^{\prime}(\rho, r)$. In the hyperbolic case,

$$
\left.B_{T}^{\prime}(\rho, \epsilon)\right)=a((-\epsilon, \epsilon)) n_{-}\left(\left(-e^{-T / 2} \epsilon, e^{-T / 2} \epsilon\right)\right) n_{+}\left(\left(-e^{-T / 2} \epsilon, e^{-T / 2} \epsilon\right)\right) .
$$

Thus, the Bowen ball in the symmetric case (in constant curvature) is a ball (or cube) of radius $r e^{-T}$ in the transverse direction to the geodesic flow. The length along the flow is not important.

11.4. Brin-Katok local entropy. Kolmogorov-Sinai entropy of an invariant measure is related to the local dimension of the measure on Bowen balls. This is the approach in Bourgain-Lindenstrauss [BL] and in [LIND], and stems from work of Young and Ledrappier-Young.

Define the local entropy on an invariant measure $\mu$ for a map $f$ by

$$
h_{\mu}(f, x)=\lim _{\delta \rightarrow 0} \limsup _{n \rightarrow \infty} \frac{-\log \mu\left(B_{n}^{f}(x, \delta)\right)}{n}=\lim _{\delta \rightarrow 0} \liminf _{n \rightarrow \infty} \frac{-\log \mu\left(B_{n}^{f}(x, \delta)\right)}{n} .
$$

Brin-Katok proved that both limits exist, that the local entropy $h_{\mu}(f, x)$ is $f$ invariant and

$$
h_{\mu}(f)=\int_{X} h_{\mu}(f, x) d \mu(x) .
$$

The definition and result is similar in the geodesic flow case. The local entropy of $\mu$ at $\rho$ is defined by,

$$
\begin{aligned}
\lim _{\epsilon \rightarrow 0} \liminf _{T \rightarrow \infty}-\frac{1}{T} \log \mu\left(B_{T}(\rho, \epsilon)\right) \\
\quad=\lim _{\epsilon \rightarrow 0} \limsup _{T \rightarrow \infty}-\frac{1}{T} \log \mu\left(B_{T}(\rho, \epsilon)\right):=h_{K S}(\mu, \rho) .
\end{aligned}
$$


11.5. Ergodic decomposition. Let $\nu$ be an invariant measure. It may be expressed as a convex combination of ergodic measures, which are extreme points of the compact convex set of invariant measures. There is a concrete formula,

$$
\nu=\int \nu_{x}^{\mathcal{E}} d \nu(x)
$$

for its ergodic decomposition, where $\nu_{x}^{\mathcal{E}}$ is the orbital average through $x \in$ $G / \Gamma$. It is a fact that $\nu_{x}^{\mathcal{E}}$ ergodic for $\nu$-a.e. $x$. Then the local entropy is

$$
h_{a(t)}\left(\tilde{\mu}_{x}^{\mathcal{E}}\right)=\lim _{\epsilon \rightarrow 0} \frac{\tilde{\mu}(T(x, \epsilon))}{\log \epsilon}, \text { a.s. } \tilde{\mu} .
$$

The Brin-Katok theorem states that the global KS entropy is

$$
h(\nu)=\int h\left(\nu_{x}^{\mathcal{E}}\right) d \nu(x) .
$$

11.6. Topological entropy of an invariant subset. Anantharaman's first entropy bound refers to the topological entropy of an invariant set $F$. Then, by definition,

$$
\begin{gathered}
h_{\text {top }}(F) \leq \lambda \Longleftrightarrow \forall \delta>0, \exists C>0: F \text { can be covered by at most } \\
C e^{n(\lambda+\delta)} \text { cylinders of length } n, \forall n .
\end{gathered}
$$

11.7. Bourgain-Lindenstrauss entropy bound. Before discussing the work of Anantharman-Nonnenmacher, we review a simpler entropy bound of Bourgain-Lindenstrauss. After initial work of Wolpert [W3], Bourgain-Lindenstrauss [BL] obtained a strong lower bound on entropies of quantum limit measures associated to Hecke eigenfunctions. It was an ingredient in Lindenstrauss' QUE theorem [LIND]. It is simple to state and illustrates the use of the local entropy and local dimensions to measure the KS entropy.

The setting is a compact or finite area arithmetic hyperbolic quotient. We have not reviewed local entropy in the non-compact finite area case, but proceed by analogy with the compact case. Then

$$
\left.B\left(\epsilon, \tau_{0}\right)=a\left(\left(-\tau_{0}, \tau_{0}\right)\right) n_{-}(\epsilon, \epsilon)\right) n_{+}((-\epsilon, \epsilon))
$$

is a Bowen ball around the identity element and $x B\left(\epsilon, \tau_{0}\right)$ is its left translate by $x \in G$. Here $\epsilon=r e^{-T / 2}$.

TheOREM 11.1. [BL] Let $\Gamma \subset S L(2, \mathbb{Z})$ be a congruence subgroup. Let $\mu$ be a quantum limit. Then for any compact subset $K \subset \Gamma \backslash G$ and any $x \in K$,

$$
\mu\left(x B\left(\epsilon, \tau_{0}\right)\right) \leq \epsilon^{2 / 9} .
$$


In the flow-box notation above,

$$
\mu_{n}\left(B_{T}(\rho, \epsilon)\right) \leq C e^{-T / 9}
$$

This implies that any ergodic component of any quantum limit has entropy $\geq \frac{1}{9}$.

Corollary 11.2. [BL] Almost every component of a quantum limit measure has entropy $\geq 1 / 9$. The Hausdorff dimenson of the support of the limit measure is $\geq 1+1 / 9$.

Theorem 11.1 is a consequence of the following uniform upper bound for masses in small tubes around geodesic segments in configuration space:

TheOREM 11.3. [BL] Let $\Gamma \subset S L(2, \mathbb{Z})$ be a congruence subgroup. Let $\mu$ be a quantum limit. Then for any compact subset $K \subset \Gamma \backslash G$ and any $x \in K$, and for any Hecke eigenfunction $\varphi_{\lambda_{j}}$,

$$
\int_{x B\left(r, \tau_{0}\right)}\left|\varphi_{\lambda_{j}}\right|^{2} d V \leq r^{2 / 9} .
$$

One may rewrite this result in terms of a Riesz-energy of the quantum limit measures:

Corollary 11.4. Let $\mu$ be a quantum limit of a sequence of HeckeMaass eigenfunctions. Then for $\kappa<2 / 9$,

$$
\int_{M} \int_{M} \frac{d \mu(x) d \mu(y)}{d_{M}(x, y)^{\kappa+1}}<\infty .
$$

We briefly sketch the idea of the proof since it makes an interesting contrast to the Anantharaman-Nonnenmacher bound. They prove that there exists a set $W$ of integers of size $\epsilon^{-2 / 9}$ so that for $n \in W$ one has (roughly) that the translates $y B\left(\epsilon, \tau_{0}\right)$ of the small balls by $y$ in the Hecke correspondence for $T_{n}$ are all pairwise disjoint. Since

$$
\left|\varphi_{\lambda}(y)\right|^{2} \leq \sum_{z \in T_{n}(y)}\left|\varphi_{\lambda}(z)\right|^{2}
$$

one has

$$
\begin{aligned}
\int_{x B\left(\epsilon, \tau_{0}\right)}\left|\varphi_{\lambda}(y)\right|^{2} d V & \leq C \int_{x B\left(\epsilon, \tau_{0}\right)} \sum_{z \in T_{n}(y)}\left|\varphi_{\lambda}(z)\right|^{2} d V \\
& =\sum_{z \in T_{n}(x)} \int_{z B\left(\epsilon, \tau_{0}\right)}\left|\varphi_{\lambda}(z)\right|^{2} d V
\end{aligned}
$$


Now sum over $n \in W$ and use the disjointness of the small balls $z B\left(\epsilon, \tau_{0}\right)$ and the $L^{2}$ normalization of the eigenfunction to obtain

$$
\begin{aligned}
\int_{x B\left(\epsilon, \tau_{0}\right)}\left|\varphi_{\lambda}(y)\right|^{2} d V & \leq \frac{1}{|W|} \sum_{n \in W} \sum_{z \in T_{n}(x)} \int_{z B\left(\epsilon, \tau_{0}\right)}\left|\varphi_{\lambda}(z)\right|^{2} d V \\
& \leq C \epsilon^{2 / 9} \int_{X} \mid \varphi_{\lambda}^{2} d V \leq C \epsilon^{2 / 9}
\end{aligned}
$$

\subsection{Anantharman and Anantharaman-Nonnenacher lower} bound. We now turn to the lower bounds on entropy in the general Anosov case given in $[\mathbf{A}]$ and in $[\mathbf{A N}, \mathbf{A N K}]$. The mechanisms proving the lower bounds and the results are somewhat different between the two articles. The result of $[\mathbf{A}]$ gives a lower bound for the topological entropy $h_{\text {top }}\left(\operatorname{supp}_{0}\right)$ of the support of a quantum limit while the main result of $[\mathbf{A N}]$ gives a lower bound on the metric or Kolmogorov Sinai entropy $h_{g}=h_{K S}$ of the quantum limits. For the definition of $h_{t o p}(F)$, see (102).

THEOREM 11.5. [A] Let $\nu_{0}=\int_{S^{*} M} \nu_{0}^{x} d \nu_{0}(x)$ be the ergodic decomposition of $\nu_{0}$. Then there exists $\kappa>0$ and two continuous decreasing functions $\tau:[0,1] \rightarrow[0,1]$ and $\vartheta:(0,1] \rightarrow \mathbb{R}_{+}$with $\tau(0)=1, \vartheta(0)=\infty$, such that

$$
\nu_{0}\left[\left\{x: h_{g}\left(\nu_{0}^{x}\right) \geq \frac{\Lambda}{2}(1-\delta)\right\}\right] \geq\left(\frac{\kappa}{\vartheta(\delta)}\right)^{2}(1-\tau(\delta)) .
$$

Hence

- $h_{g}\left(\nu_{0}\right)>0$

- $h_{\text {top }}\left(\operatorname{supp} \nu_{0}\right) \geq \frac{\Lambda}{2}$.

It follows that a positive proportion of the ergodic components of $\nu_{0}$ must have KS entropy arbitrarily close to $\frac{\Lambda}{2}$.

This is proved as a consequence of a Proposition which may have other applications:

THEOREM 11.6. [A] (Proposition 2.0.4) With the same notation as in Theorem 11.5 and Remark 11.10. Let $F$ be a subset with $h_{\text {top }}(F) \leq \frac{\Lambda}{2}(1-\delta)$. Then,

$$
\nu_{0}(F) \leq\left(1-\left(\frac{\kappa}{\vartheta(\delta)}\right)^{2}\right)(1-\tau(\delta))<1 .
$$

Hence supp $\nu_{0}$ cannot equal $F$.

In [A] Theorem 1.1.2, there is a generalization to quasi-modes which introduces a constant $c$. For simplicity we only consider eigenfunctions.

In the subsequent article of Anantharaman-Nonnenmacher, $[\mathbf{A N}, \mathbf{A N K}]$ the authors obtain a quantitative lower bound on the KS entropy: 
TheOREM 11.7. [AN, ANK] Let $\mu$ be a semiclassical measure associated to the eigenfunctions of the Laplacian on $M$. Then its metric entropy satisfies

$$
h_{K S}(\mu) \geq\left|\int_{S^{*} M} \log J^{u}(\rho) d \mu(\rho)\right|-\frac{(d-1)}{2} \lambda_{\max },
$$

where $d=\operatorname{dim} M . \quad \lambda_{\max }=\lim _{|t| \rightarrow \infty} \frac{1}{t} \log \sup _{\rho \in \mathcal{E}}\left|d g_{\rho}^{t}\right|$ is the maximal expansion rate of the geodesic flow on $\mathcal{E}(5)$ and $J^{u}$ is the unstable Jacobian determinant (3).

When $M$ has constant sectional curvature -1 , the theorem implies that

$$
h_{K S}(\mu) \geq \frac{d-1}{2} .
$$

They state the conjecture

ConjeCture 11.8. [AN] Let $(M, g)$ have Anosov geodesic flow. Then for any quantum limit measure $\nu_{0}$,

$$
h_{g}\left(\nu_{0}\right) \geq \frac{1}{2}\left|\int_{S^{*} M} \log J^{u}(v) d \nu_{0}(v)\right| .
$$

This conjecture has recently been proved by G. Rivière for surfaces of negative curvature $[\mathbf{R i v}]$.

This conjecture does not imply QUE. In fact the counterexamples in $[\mathbf{F N B}, \mathbf{F N}]$ to QUE in the case of "quantum cat maps" satisfy the condition of the conjecture. So there is some chance that the conjecture is best possible for $(M, g)$ with ergodic geodesic flow.

To the author's knowledge, the corollary that quantum limits in the Anosov case cannot be pure periodic orbit measures has not been proved a simpler way that by applying the theorems above. In $\S 11.21$ we will go over the proof in that special case and see how it ties together with mass concentration of eigenfunctions around hyperbolic closed geodesics in $\S 5$.

11.9. Problem with semi-classical estimates and Bowen balls. As a first attempt to estimate KS entropies of quantum limits, one might try to study the local entropy formula as in Bourgain-Lindenstrauss. Let $B_{T}(\rho, \epsilon)$ be the small tube around $\rho \in S^{*} M$, i.e. of length $\epsilon$ in the flow direction and $e^{-T / 2}$ in the stable/unstable directions. As in the BourgainLindenstrauss bound, one would like to understand the decay of $\mu_{n}\left(B_{T}(\rho, \epsilon)\right)$ as $T \rightarrow \infty$ and $n \rightarrow \infty$. In the brief expository article [AN2], the authors give the heuristic estimate

$$
\mu_{n}\left(B_{T}(\rho, \epsilon)\right) \leq C \lambda_{n}^{\frac{d-1}{2}} e^{-\frac{(d-1) T}{2}}, \quad d=\operatorname{dim} M
$$

on the local dimensions of the quantum measures for a sequence of eigenfunctions. Unlike the uniform Bourgain-Lindenstrauss estimate, the estimate is $\lambda_{n}$-dependent and is only non-trivial for times $T>\log \lambda_{n}$. But then the ball 
radius (in the stable/unstable directions) is $e^{-T}=\lambda_{n}^{-1 / 2}$, which is just below the minimal scale allowed by the uncertainty principle (\$1.4). To obtain a useful entropy bound one needs to take $T=2 \log \lambda_{n}$ and then $e^{-T}=\lambda_{n}^{-1}$, well below the Heisenberg uncertainty threshold. So although it is attractive, the local entropy is difficult to use. Recall that Bourgain-Lindenstrauss used the many elements of the Hecke correspondences to move the small ball around so that it almost fills out the manifold, and then used the $L^{2}$ normalization to estimate the total mass. There doesn't seem to exist a similar mechanism to build up a big mass from the small balls in the general Anosov case.

11.10. Some important parameters. Two important parameters $\kappa$, $\vartheta$ appear here and in the statements and proofs in $[\mathbf{A}]$. They represent time scales relative to the Ehrenfest time:

- $\kappa$ : Semi-classical estimates only work when $n \leq \kappa|\log \hbar|$, i.e. $\kappa|\log \hbar|$ plays the role of the Ehrenfest time.

- $\vartheta$ : The main estimate is only useful when $h^{-d / 2} e^{-n \Lambda / 2}<<1$ or $n \geq \vartheta|\log \hbar|$.

11.11. Cylinder set operators in quantum mechanics. To get out of the too-small Bowen ball impasse, Anantharaman $[\mathbf{A}]$ and AnanthramanNonnenmacher study "quantum measures" of cylinder sets $\mathcal{C}$ rather than small balls. The emphasis (and results) on quantum cylinder sets is one of the key innovations in $[\mathbf{A}]$. The classical cylinder sets are not directly involved in the main estimates of $[\mathbf{A}, \mathbf{A N}]$. Rather they are quantized as cylinder set operators. Then their "quantum measure" or quantum entropy is studied. To distinguish notationally between classical and quantum objects, we put a $\hat{\imath}$ on the quantum operator.

To define quantum cylinder set operators, one quantizes the partition to define a smooth quantum partition of unity $\hat{P}_{k}$ by smoothing out the characteristic functions of $M_{k}$. Let $\alpha=\left[\alpha_{0}, \ldots, \alpha_{n-1} \in \Sigma_{n}\right.$ be a cylinder set in sequence space $\Sigma$. The corresponding quantum cylinder operator is

$$
\hat{P}_{\alpha}=\hat{P}_{\alpha_{n-1}}(n-1) \hat{P}_{\alpha_{n}-2}(n-2) \cdots \hat{P}_{\alpha_{0}},
$$

where $\alpha=\left[\alpha_{0}, \ldots, \alpha_{n-1}\right]$ where

$$
\hat{P}(k)=U^{* k} \hat{P} U^{k} .
$$

Here, $U=e^{i \sqrt{\Delta}}$ is the propagator at unit time (or in the semi-classical framework, $U=e^{i \hbar \Delta / 2}$ (see [A] (1.3.4)).

The analogue of the measure of a cylinder set in quantum mechanics is given by the matrix element of the cylinder set operator in the energy state, transported to $\Sigma$. We use the semi-classical notation of $[\mathbf{A}]$, but it is easily converted to homogeneous notation. 
Definition: (See $[\mathbf{A}],(1.3 .4)$ ) Let $\psi_{\hbar}$ be an eigenfunction of $\Delta$. Define the associated quantum "measure" of cylinder sets $\mathcal{C}=\left[\alpha_{0}, \ldots, \alpha_{n-1}\right] \in \Sigma_{n}$ by

$$
\mu_{\hbar}\left(\left[\alpha_{0}, \ldots, \alpha_{n}\right]\right)=\left\langle\hat{P}_{\alpha_{n}}(n) \cdots \hat{P}_{\alpha_{0}}(0) \psi_{\hbar}, \psi_{\hbar}\right\rangle
$$

Thus, one "quantizes" the cylinder set $\mathcal{C}=\left[\alpha_{0}, \ldots, \alpha_{n-1}\right]$ as the operator

$$
\hat{\mathcal{C}}=U^{-(n-1)} \hat{P}_{\alpha_{n-1}} U \hat{P}_{\alpha_{n-2}} \cdots U \hat{P}_{\alpha_{0}}
$$

For each eigenfunction, one obtains a linear functional

$$
\mu_{\hbar}\left(\left[\alpha_{0}, \ldots, \alpha_{n-1}\right]\right)=\rho_{\hbar}(\hat{\mathcal{C}})
$$

in the notation of states of $\S 3$. The "quantum measures" are shift invariant, i.e.

$$
\mu_{\hbar}\left(\left[\alpha_{0}, \ldots, \alpha_{n-1}\right]\right)=\mu_{\hbar}\left(\sigma^{-1}\left[\alpha_{0}, \ldots, \alpha_{n-1}\right]\right)
$$

This says,

$$
\begin{aligned}
& \left\langle U^{-(n-2)} P_{\alpha_{n-1}} U^{n-2} \cdots U P_{\alpha_{0}} U^{-1} \varphi_{\hbar}, \varphi_{\hbar}\right\rangle \\
& =\left\langle U^{-(n-1)} P_{\alpha_{n-1}} U^{n-1} \cdots U P_{\alpha_{0}} \varphi_{\hbar}, \varphi_{\hbar}\right\rangle
\end{aligned}
$$

which is true since $\rho_{h}(A)=\left\langle A \varphi_{\hbar}, \varphi_{\hbar}\right\rangle$ is an invariant state.

The matrix element $\left\langle\hat{P}_{\alpha} \psi_{\hbar}, \psi_{\hbar}\right\rangle$ is the probability (amplitude) that the particle in state $\psi_{\hbar}$ visits the phase space regions $P_{\alpha_{0}}, P_{\alpha_{1}}, \ldots, P_{\alpha_{n-1}}$ at times $0,1, \ldots, n-1$.

Thus, the states $\rho_{\hbar}$ or alternatively the Wigner distributions of $\psi_{\hbar}$ are transported to $\Sigma$ to define linear functionals $\mu_{\hbar}$ on the span of the cylinder functions on $\Sigma$. They exactly invariant under the shift map.

The functionals $\mu_{\hbar}$ are not positive measures since $\hat{\mathcal{C}}_{\hbar}$ is not a positive operator. However as in the proof of the quantum ergodicity Theorem 4.1, one has the Schwartz inequality,

$$
\left|\rho_{\hbar}(\hat{\mathcal{C}})\right|^{2} \leq \rho_{\hbar}\left(\hat{\mathcal{C}}^{*} \hat{\mathcal{C}}\right)
$$

reflecting the positivity of the state $\rho_{\hbar}$.

11.12. Main estimate for cylinder sets of $\Sigma$. The so-called main estimate of $[\mathbf{A}]$ implies that for every cylinder set $\mathcal{C} \in \Sigma_{n}$, one has

$$
\left|\mu_{\hbar}(\mathcal{C})\right| \leq C_{\beta} h^{-d / 2} e^{-n \Lambda / 2}(1+O(\epsilon))^{n}, \quad \text { uniformly for } n \leq \beta|\log \hbar| .
$$

Here, $\beta$ can be taken arbitrarily large. This estimate is similar in spirit to (106) but circumvents the uncertainty principle. It is one of the essential quantum mechanical (or semi-classical) ingredient for getting a lower bound on $h_{t o p}\left(s u p p \nu_{0}\right)$ of supports of quantum limit measures (see $\left.\S 11.15\right)$. 


\subsection{Quantization of cylinder sets versus quantized cylinder} operators. Let us explain how (113) gets around the uncertainty principle. A fundamental feature of quantization is that it is not a homomorphism. Thus, the quantization of the (smoothed) characteristic function of the cylinder set $P_{\alpha_{0}} \cap g^{-1} P_{\alpha_{1}} \cap \cdots \cap g^{-n} P_{\alpha_{n}}$ is very different from $\hat{P}_{\alpha_{n-1}} U \hat{P}_{\alpha_{n-2}} \ldots$ $U \hat{P}_{\alpha_{0}}$, the ordering which occurs in the quantum cylinder operator $\hat{P}_{\alpha}$ in (107). The problematic Bowen ball estimate (106) would arise if one quantized the Bowen ball (or cylinder set) in the first sense and then took its matrix element. Quantizing in the other order makes the main estimate possible.

11.14. Quantized cylinder operators and covering numbers. As motivation to study quantum measures of cylinder sets, Anantharaman observes that the main estimate suggests an obstruction to a quantum measure concentrating on a set $F$ with $h_{t o p}(F)<\frac{\Lambda}{2}$. For each $n$ such a set can be covered by $C e^{n\left(\frac{\Lambda}{2}(1-\delta)\right.}$ cylinder sets of length $n$. By the main estimate (113), each of the cylinder sets has quantum measure $\left|\mu_{\hbar}(\mathcal{C})\right| \leq$ $C e^{-d / 2 \log \hbar-n \Lambda / 2} \quad$ uniformly for $n \leq \kappa|\log \hbar|$ for any $\kappa$. As yet, the combination does not disprove that $\operatorname{supp} \nu_{0}=F$, i.e. $\nu_{0}(F)=1$, since the large factor of $h^{-d / 2}$ is not cancelled in the resulting estimate $\mu_{\hbar}(F) \leq$ $C e^{n\left(\frac{\Lambda}{2}(1-\delta)-d / 2 \log \hbar-n \Lambda / 2\right.}$. An additional idea is needed to decouple the large time parameter $n$ into independent time parameters. Roughly speaking, this is done by a sub-multiplicative property. In $\S 11.20$, the ideas in the above paragraph are made more precise and effective.

11.15. Upper semi-continuity, sub-multiplicativity and subadditivity. Before getting into the details of (the sketch of) the proof, it is useful to consider why there should exist a non-trivial lower bound on $h_{K S}\left(\nu_{0}\right)$ for a quantum limit measure? What constrains a quantum limit measure to have higher entropy than some given invariant measure?

First, the classical entropy of an invariant is upper semi-continuous with respect to weak convergence. This is in the right direction for proving lower entropy bounds for quantum limits. If one can obtain a lower bound on the "entropies" of the quantum measures $\mu_{n}$, then the entropy of the limit can only jump up. Of course, the quantum measures are not $g^{t}$ invariant measures and so upper semi-continuity does not literally apply. One needs to define a useful notion of entropy for the quantum measures and prove some version of upper semi-continuity for it. The USC property of the classical entropy suggests that there should exist some analogue on the quantum level.

In $[\mathbf{A}]$, entropies are studied via special covers by cylinder sets. For these, there is a sub-multiplicative estimate on the number of elements in the cover.

In $[\mathbf{A N}]$, a quite different idea is used: the authors employ a notion of quantum entropy due to Maassen-Uffink and prove a certain lower bound 
for the quantum entropy called the entropy uncertainty inequality. It is the origin of the lower bound for the quantum entropies and the KS entropy in Theorem 11.7 (see $\$ 11.17$ ). As a replacement for the USC property of the entropy, a certain sub-additivity property for the quantum entropy is proved (see $\S 11.18)$.

In the next three subsections, we go over the main ingredients in the proof. Then we give a quick sketch of the full proof.

11.16. Main estimate in more detail. The main estimate in $[\mathbf{A}]$ (Theorem 1.3.3) was refined in $[\mathbf{A N}, \mathbf{A N K}]$. To state the results, we need some further notation. Let $n_{E}(\hbar)$ denote the Ehrenfest time

$$
n_{E}(\hbar)=\left[\frac{(1-\delta)|\log \hbar|}{\lambda_{\max }}\right]
$$

where the brackets denote the integer part and $\delta$ is a certain small number arising in energy localization (for the definition of $\lambda_{\max }$ see (5)). The authors also introduce a discrete "coarse-grained" unstable Jacobian

$$
J_{1}^{u}\left(\alpha_{0}, \alpha_{1}\right):=\sup \left\{J^{u}(\rho): \rho \in T^{*} \Omega_{\alpha_{0}} \cap \mathcal{E}^{\epsilon}: g^{t} \rho \in T^{*} \Omega_{\alpha_{1}}\right\},
$$

for $\alpha_{0}, \alpha_{1}=1, \ldots, K$. Here, $\Omega_{j}$ are small open neighborhoods of the partition sets $M_{j}$. For a sequence $\alpha=\left(\alpha_{0}, \ldots, \alpha_{n-1}\right)$ of symbols of length $n$, one defines

$$
J_{n}^{u}(\alpha):=J_{1}^{u}\left(\alpha_{0}, \alpha_{1}\right) \cdots J_{1}^{u}\left(\alpha_{n-2}, \alpha_{n-1}\right) .
$$

THEOREM 11.9. (See Theorem 3.5 of $[\mathbf{A N K}]$ ) Given a partition $\mathcal{P}^{(0)}$ and $\delta, \delta^{\prime}>0$ small enough, there exists $\hbar_{\mathcal{P}^{(0)}, \delta, \delta^{\prime}}$ such that, for any $\hbar \leq \hbar_{\mathcal{P}^{(0)}, \delta, \delta^{\prime}}$, for any positive integer $n \leq n_{E}(\hbar)$, and any pair of sequences $\alpha$, $\alpha^{\prime}$ of length $n$,

$$
|| \hat{P}_{\alpha^{\prime}}^{*} U^{n} \hat{P}_{\alpha} \mathrm{Op}\left(\chi^{(n)}\right)|| \leq C \hbar^{-(d-1+c \delta)} \sqrt{J_{n}^{u}(\alpha) J_{n}^{u}\left(\alpha^{\prime}\right)} .
$$

Here, $d=\operatorname{dim} M$ and the constants $c, C$ only depend on $(M, g)$.

There is a more refined version using a sharper energy cutoff in $[\mathbf{A N}]$.

THEOREM 11.10. Given a partition $\mathcal{P}^{(0)}, \kappa>0$ and $\delta>0$ small enough, there exists $\hbar_{\mathcal{P}^{(0)}, \delta, \kappa}$ such that, for any $\hbar \leq \hbar_{\mathcal{P}^{(0)}, \delta, \kappa}$, for any positive integer $n \leq \kappa|\log \hbar|$, and any pair of sequences $\alpha$, $\alpha^{\prime}$ of length $n$,

$$
|| \hat{P}_{\alpha^{\prime}}^{*} U^{n} \hat{P}_{\alpha} \operatorname{Op}\left(\chi^{(n)}\right)|| \leq C \hbar^{-\left(\frac{d-1}{2}+\delta\right)} e^{-(d-1) n}\left(1+O\left(\hbar^{\delta}\right)\right)^{n} .
$$

Here, $d=\operatorname{dim} M$.

To prove this, one shows that any state of the form $\mathrm{Op}\left(\chi^{(*)}\right) \Psi$ can be decomposed as a superposition of essentially $\hbar^{-\frac{(d-1)}{2}}$ normalized Lagrangian states, supported on Lagrangian manifolds transverse to the stable leaves of the flow. The action of the operator $\hat{P}_{\alpha}$ on such Lagrangian states is intuitively as follows: each application of $U$ stretches the Lagrangian in the 
unstable direction (the rate of elongation being described by the unstable Jacobian) whereas each multiplication by $\hat{P}_{\alpha_{j}}$ cuts off the small piece of the Lagrangian in the $\alpha$ th cell. This iteration of stretching and cutting accounts for the exponential decay. A somewhat more detailed exposition is in $\$ 11.22$.

This estimate can be reformulated as a statement about matrix elements of the operators $\hat{P}_{\alpha}$ relative to eigenfunctions (see Theorem 3.1 of $[\mathbf{A N K}]$ )

TheOREM 11.11. Let $\hat{P}_{k}=\mathbf{1}_{M_{k}}^{\text {sm }}$. For any $\kappa>0$ there exists $\hbar_{\kappa}>0$ so that, uniformly for $\hbar<\hbar_{\kappa}$ and all $n \leq \kappa|\log \hbar|$, and for all $\alpha=$ $\left(\alpha_{0}, \ldots, \alpha_{n-1}\right) \in[1, \kappa]^{n} \cap \mathbb{Z}^{n}$,

$$
\left\|\hat{P}_{\alpha} \psi_{h}\right\| \leq 2(2 \pi \hbar)^{-d / 2} e^{-n \frac{\Lambda}{2}}(1+O(\epsilon))^{n} .
$$

The cutoff operator $O p\left(\chi^{(n)}\right)$ is supported near the energy surface $\lambda_{n}^{2}$, and the eigenfunctions are supported on their energy surfaces. Here, $\Lambda$ is the "smallest" expansion rate (see (4)).

We recall that $\left\langle\hat{P}_{\alpha} \psi_{\hbar}, \psi_{\hbar}\right\rangle$ is the probability (amplitude) that the particle in state $\psi_{\hbar}$ visits the phase space regions $P_{\alpha_{0}}, P_{\alpha_{1}}, \ldots, P_{\alpha_{n-1}}$ at times $0,1, \ldots, n-1$. The main estimates show that this probability decays exponentially fast with $n$, at rate $\frac{\Lambda}{2}$. However, the decay only starts near the Eherenfest time $n_{1}=\frac{d|\log \hbar|}{\Lambda}$.

11.17. Entropy uncertainty principle. The source of the lower bound for $h_{K S}\left(\nu_{0}\right)$ is most simply explained in $[\mathbf{A N}]$. It is based on the "entropic uncertainty principle" of Maassen- Uffink. There are several notions of quantum or non-commutative entropy, but for applications to eigenfunctions it is important to find one with good semi-classical properties.

Let $(\mathcal{H},\langle.,\rangle$.$) be a complex Hilbert space, and let \|\psi\|=\sqrt{\langle\psi, \psi\rangle}$ denote the associated norm. The quantum notion of partition is a family $\pi=$ $\left(\pi_{k}\right)_{k=1, \ldots, \mathcal{N}}$ of operators on $\mathcal{H}$ such that $\sum_{k=1}^{\mathcal{H}} \pi_{k} \pi_{k}^{*}=I d$. If $\|\psi\|=1$, the entropy of $\psi$ with respect to the partition $\pi$ is define by

$$
h_{\pi}(\psi)=-\sum_{k=1}^{\mathcal{N}}\left\|\pi_{k}^{*} \psi\right\|^{2} \log \left\|\pi_{k}^{*} \psi\right\|^{2} .
$$

We note that the quantum analogue of an invariant probability measure $\mu$ is an invariant state $\rho$, and the direct analogue of the entropy of the partition would be $\sum \rho\left(\pi_{k} \pi_{k}^{*}\right) \log \rho\left(\pi_{k} \pi_{k}^{*}\right)$. If the state is $\rho(A)=\langle A \psi, \psi\rangle$ then $\rho\left(\pi_{k} \pi_{k}^{*}\right)=\left\|\pi_{k}^{*} \psi\right\|^{2}$.

The dynamics is generated by a unitary operator $\mathcal{U}$ on $\mathcal{H}$. We now state a simple version of the entropy uncertainty inequality of Maasen-Uffink. A more elaborate version in $[\mathbf{A}, \mathbf{A N}, \mathbf{A N K}]$ gives a lower bound for a certain "pressure".

TheOREM 11.12. For any $\epsilon \geq 0$, for any normalized $\psi \in \mathcal{H}$,

$$
h_{\pi}(\mathcal{U} \psi)+h_{\pi}(\psi) \geq-2 \log c(\mathcal{U})
$$


where

$$
c(\mathcal{U})=\sup _{j, k}\left|\left\langle e_{k}, \mathcal{U} e_{j}\right\rangle\right|
$$

is the supremum of all matrix elements in the orthonormal basis $\left\{e_{j}\right\}$. In particular, $h_{\pi}(\psi) \geq-\log c(\mathcal{U})$ if $\psi$ is an eigenfunction of $\mathcal{U}$.

The intuitive idea is that if a unitary matrix has small entries, then each of its eigenvectors must have large Shannon entropy.

This uncertainty principle is applied to the entropies of the partitions defined above. For the propagator, we put $\mathcal{U}=e^{i T_{E} \sqrt{\Delta}}$ is the wave operator at the "Ehrenfest time" $T_{E}=\frac{\log \lambda}{\lambda_{\max }}$. Or in the semi-classical framework (where $h=\frac{1}{\lambda}$ ), one can use the Hamiltonian is $H=h^{2} \Delta$ and the time evolution $\mathcal{U}=e^{i n_{E}(h) h \Delta}$ with $n_{E}(h)=\frac{\log \frac{1}{h}}{\lambda_{\max }}$.

Applied to an eigenfunction of $\mathcal{U}$, one has

$$
h_{\pi}\left(\psi_{h}\right)=\sum_{|\alpha|=n_{E}}\left\|\hat{P}_{\alpha}^{*} \psi_{h}\right\|^{2} \log \left\|\hat{P}_{\alpha}^{*} \psi_{h}\right\|^{2},
$$

and obviously

$$
h_{\pi}(\mathcal{U} \psi)+h_{\pi}(\psi)=2 h_{\pi}(\psi)
$$

On the right side,

$$
c(\mathcal{U})=\max _{|\alpha|=\left|\alpha^{\prime}\right|=n_{E}}\left\|\hat{P}_{\alpha^{\prime}} U^{n_{E}} \hat{P}_{\alpha} O p\left(\chi^{\left(n_{E}\right)}\right)\right\|
$$

where $\chi^{\left(n_{E}\right)}$ is a very sharp energy cutoff supported in a tubular neighborhood $\mathcal{E}^{\epsilon}:=H^{-1}(1-\epsilon, 1+\epsilon)$ of $\mathcal{E}=S^{*} M$ of width $2 h^{1-\delta} e^{n \delta}$ for a given $\delta>0$.

11.18. Sub-additivity. Another key component in the proof is that the quantum entropy is almost sub-additive for $\hbar \leq|\log t|$.

Sub-additivity of the classical KS entropy follows from the concavity of the function $-x \log x$. It states that the sequence $\left\{h_{n}(\mu, P)\right\}$ is sub-additive, i.e.

$$
h_{n+m}(\mu, P) \leq h_{n}(\mu, P)+h_{m}(\mu, P) .
$$

However, this is false for the quantum measures.

The correct statement is as follows: There exists a function $R\left(n_{0}, \hbar\right)$ such that $\lim _{\hbar \rightarrow 0} R\left(n_{0}, \hbar\right)$ for all $n_{0} \in \mathbb{Z}$ and such that for all $n_{0}, n \in \mathbf{N}$ with $n_{0}+n=\frac{(1-\delta)|\log \hbar|}{\lambda_{\max }}$, and for any normalized eigenfunction $\varphi_{\lambda}$, one has

$$
h_{\mathcal{P}^{n_{0}+n}}\left(\varphi_{\lambda}\right) \leq h_{\mathcal{P}^{n_{0}}}\left(\varphi_{\lambda}\right)+h_{\mathcal{P}^{n}}\left(\varphi_{\lambda}\right)+R\left(n_{0}, \hbar\right) .
$$


11.19. Outline of proof of Theorem 11.7. We outline the proof from $[\mathbf{A N}]$. Let

$$
h_{\mathcal{P}^{(n)}}(\psi)=-\sum_{|\alpha|=n}\left\|\hat{P}_{\alpha}^{*} \psi\right\|^{2} \log \left\|\hat{P}_{\alpha}^{*} \psi\right\|^{2}
$$

and let

$$
h_{\mathcal{T}^{(n)}}(\psi)=-\sum_{|\alpha|=n}\left\|\hat{P}_{\alpha} \psi\right\|^{2} \log \left\|\hat{P}_{\alpha} \psi\right\|^{2},
$$

(1) Let $K=\frac{1-\delta}{\lambda_{\max }}$. By the main estimate and the entropy uncertainty inequality, one has

$$
\begin{aligned}
h_{\mathcal{T}^{(n)}}\left(\psi_{h}\right)+h_{\mathcal{P}^{(n)}}\left(\psi_{h}\right) \geq & 2(d-1) n+\frac{(d-1+2 \delta) \lambda_{\max }}{(1-\delta)} n_{E}(\hbar) \\
& +O(1) .
\end{aligned}
$$

(2) Fix $n_{0}, n_{E}(\hbar)$. Write $n=q n_{0}+r, r \leq n_{0}$. Then by sub-additivity,

$$
\frac{h_{\mathcal{P}^{(n)}}\left(\psi_{h}\right)}{n} \leq \frac{h_{\mathcal{P}^{\left(n_{0}\right)}}\left(\psi_{h}\right)}{n_{0}}+\frac{h_{\mathcal{P}^{(r)}}\left(\psi_{h}\right)}{n_{E}(\hbar)}+\frac{R\left(n_{0}, \hbar\right)}{n_{0}} .
$$

(3) As $\hbar \rightarrow 0$, this gives

$$
\begin{aligned}
\frac{h_{\mathcal{P}^{\left(n_{0}\right)}}\left(\psi_{h}\right)}{n_{0}}+\frac{h_{\mathcal{T}^{\left(n_{0}\right)}}\left(\psi_{h}\right)}{n_{0}} \geq & 2(d-1) n+\frac{(d-1+2 \delta) \lambda_{\max }}{(1-\delta)} n \\
& +O(1)-\frac{R\left(n_{0}, \hbar\right)}{2 n_{0}}+O_{n_{0}}(1 / n) .
\end{aligned}
$$

(4) Now take the limit $\hbar \rightarrow 0$. The expressions $\left\|\hat{P}_{\alpha} \varphi_{\lambda}\right\|^{2}$ and the Shannon entropies tend to their classical values. Hence, $h_{\mathcal{P}^{\left(n_{0}\right)}}\left(\psi_{h}\right)$ tends to the KS entropy of the quantum limit. Thus, the lower bound implies

$$
\frac{h_{n_{0}}(\mu)}{n_{0}} \geq d-1-\frac{(d-1+2 \delta) \lambda_{\max }}{2(1-\delta)} .
$$

Since $\delta, \delta^{\prime}$ are arbitrary one can set them equal to zero. Then let $n_{0} \rightarrow \infty$ to obtain the KS entropy of the quantum limit.

11.20. Sketch of proof of Theorem $11.5[\mathbf{A}]$. The proof of Theorem 11.5 is less structural than the proof of Theorem 11.7. There is no magic weapon like the entropic uncertainty principle. In its place there is the construction of special covers by cylinder sets and counting arguments for the number of elements in the cover. These covering arguments probably have further applications and (although quantum) seem more geometric than the argument based on the entropic uncertainty principle.

We mainly discuss the statement that $h_{t o p}\left(\operatorname{supp} \nu_{0}\right) \geq \frac{\Lambda}{2}$. We recall (§11.6) that an invariant set $F$ is with $h_{\text {top }}(F) \leq \frac{\Lambda}{2}(1-\delta)$ can be covered 
by at $C e^{N\left(\frac{\Lambda}{2}(1-\delta)\right.}$ cylinder sets of length $N$. We further recall that, by the main estimate (113), each of the cylinder sets has quantum measure $\left|\mu_{\hbar}(\mathcal{C})\right| \leq C e^{-d / 2 \log \hbar-N \Lambda / 2} \quad$ uniformly for $N \leq \vartheta|\log \hbar|$ for any $\vartheta$. We want to combine these estimates to show that $\nu_{0}(F)<1$ for any quantum limit $\nu_{0}$. But we cannot simply multiply the measure estimate for $\mathcal{C}$ in the cover times the number of cylinder sets in the cover. The main estimate being fixed, the only things left to work with are the covers.

As will be seen below, one needs to introduce special covers adapted to the eigenfunctions. The covering number of such covers has a submultiplicative property. We motivate it by going through a heuristic proof from $[\mathbf{A}]$. We also add a new step that helps explain why special covers are needed. See also $\S 11.21$ where the pitfalls are explored when $F=\gamma$ (a closed hyperbolic orbit).

11.20.1. Covers and times, $I$. Let $F \subset \Sigma$ be a shift-invariant subset with $h_{\text {top }}(F) \leq \frac{\Lambda}{2}(1-\delta)$. Let $W_{n} \subset \Sigma_{n}$ be a cover of minimal cardinality of $F$ by $n$ cylinder-sets. Now introduce a new time $N>>n$ and define

$$
\Sigma_{N}\left(W_{n}, \tau\right):=\left\{\begin{array}{l}
\left\{N-\text { cylinders }\left[\alpha_{0}, \ldots, \alpha_{N-1}\right]\right. \text { such that } \\
\frac{\#\left\{j \in[0, N-n]:\left[\alpha_{j}, \ldots, \alpha_{j+n-1}\right] \in W_{n}\right\}}{N-n+1} \geq \tau .
\end{array}\right.
$$

They correspond to orbits that spend a proportion $\geq \tau$ of their time in $W_{n}$. In terms of the (ergodic) Liouville measure (transported to $\Sigma$ ), for almost every point of $S^{*} M$ (or its image under $I$ ), the proportion of the total time its orbit spends in $W_{n}$ is the relative measure of (the union of the cylinder sets in) $W_{n}$. If this measure is smaller than $\tau$, then $\Sigma_{N}\left(W_{n}, \tau\right)$ is a rare event as $N \rightarrow \infty$. Its cylinders satisfy $\sigma^{j}(\mathcal{C}) \cap W_{n}=\mathcal{C}$ for a proportion $\tau$ of $0 \leq j \leq N$. If one imagines partitioning the elements of $W_{n}$ into $N$ cylinders, then only a (relatively) sparse subset will go into $\Sigma_{N}\left(W_{n}, \tau\right)$. The next Lemma gives an upper bound on that number:

LEMmA 11.13. (see Lemma 2.3.1 of $[\mathbf{A}]$ ) For all $n_{0}$ there exist $n \geq n_{0}$ and $N_{0}$ so that for $N \geq N_{0}$ and $\tau \in[0,1]$, the cover $W_{n}$ of $F$ of minimal cardinality satisfies,

$$
\# \Sigma_{N}\left(W_{n}, \tau\right) \leq e^{N \frac{3 \Lambda \delta}{8}} e^{N h_{t o p}(F)} e^{(1-\tau) N(1+n) \log \ell} .
$$

where $\ell$ is the number of elements in the partition.

If $\tau$ is chosen sufficiently close to 1 , this implies:

Corollary 11.14. (See (2.3.1) of $[\mathbf{A}]$ ) If $F$ is an invariant set with $h_{\text {top }}(F)<\frac{\Lambda}{2}(1-\delta)$, then

$$
\# \Sigma_{N}\left(W_{n}, \tau\right) \leq e^{N\left(\frac{\Lambda}{2}(1-\delta)\right)} e^{(1-\tau) N(1+n) \log \ell} .
$$


We now re-write this estimate in terms of the semi-classical parameter $\hbar$. If $\tau$ is sufficiently close to 1 and $\varepsilon>0$ is sufficiently small, there exists $\vartheta>0$ so that for $N \geq \vartheta|\log \hbar|$

$$
e^{N\left(\frac{\Lambda}{2}(1-\delta)\right)} e^{(1-\tau) N(1+n) \log \ell}<(1-\varepsilon) h^{d / 2} e^{N \Lambda / 2} .
$$

Indeed, let $\alpha=-\delta \frac{\Lambda}{2}+(1-\tau)(1+n) \log \ell$. For $\tau$ very close to 1 it is negative. We then choose $\vartheta$ so that $h^{-\alpha \vartheta}<(1-\varepsilon) h^{d / 2}$, i.e. so that $|\alpha \vartheta|>d / 2$.

By the main estimate (113) and (122), we obtain the following

Corollary 11.15. (see $[\mathbf{A}]$, (2.0.1))

For $\tau$ sufficiently close to 1 and $\varepsilon$ close to zero, there exists $\vartheta>0$ so that for $N \geq \vartheta|\log \hbar|$,

$$
\left|\mu_{\hbar}\left(\Sigma_{N}\left(W_{n}, \tau\right)\right)\right| \leq 1-\varepsilon
$$

Here, $\mu_{h}\left(\Sigma_{N}\left(W_{n}, \tau\right)\right)$ means $\mu_{h}\left(\bigcup_{\left.\mathcal{C} \in \Sigma_{N}\left(W_{n}, \tau\right)\right)} \mathcal{C}\right)$.

11.20.2. Heuristic Proof. We now present a heuristic proof of Theorem 11.5 from Section 2 of $[\mathbf{A}]$ that explains the need for some rather technical machinery introduced below.

We recall that $\mu_{\hbar}$ is a shift $(\sigma)$-invariant measure on $\Sigma$. Let us temporarily pretend that $\mu_{\hbar}$ is also a positive measure. Then we would have for $N \geq \vartheta|\log \hbar|$

$$
\begin{aligned}
\left|\mu_{\hbar}\left(W_{n}\right)\right| & =\left|\frac{1}{N-n} \sum_{k=0}^{N-n-1} \mu_{\hbar}\left(\sigma^{-k} W_{n}\right)\right| \\
& =\left|\mu_{\hbar}\left(\frac{1}{N-n} \sum_{k=0}^{N-n-1} \mathbf{1}_{\sigma^{-k} W_{n}}\right)\right| \\
& \leq \sum_{\mathcal{C} \in \Sigma_{N}\left(W_{n}, \tau\right)} \mu_{\hbar}(\mathcal{C})+\tau \sum_{\mathcal{C} \notin \Sigma_{N}\left(W_{n}, \tau\right)} \mu_{\hbar}(\mathcal{C}) \\
& =\mu_{\hbar}\left(\Sigma_{N}\left(W_{n}, \tau\right)\right)+\tau \mu_{\hbar}\left(\Sigma_{N}\left(W_{n}, \tau\right)^{c}\right) \\
& \leq(1-\tau)(1-\varepsilon)+\tau .
\end{aligned}
$$

Here we used (123), and that the sum of the $\mu_{h}$ measures of $\Sigma_{N}\left(W_{n}, \tau\right)$ and its complement add to one. Since $W_{n}$ is fixed, the weak convergence $\mu_{\hbar} \rightarrow \mu_{0}$ implies,

$$
\left.\left|\mu_{0}\left(W_{n}\right)\right| \leq(1-\tau)(1-\varepsilon)\right)+\tau<1 .
$$

Since $F \subset W_{n}$, the same estimate applies to $F$. So $F$ cannot be the support of $\mu_{0}$. "QED"

In the above steps, we use that $\sigma^{-k} W_{n} \subset \Sigma_{N}\left(W_{n}, \tau\right)$ for $k \leq N-n-1$ and that

$$
\frac{1}{N-n} \sum_{k=0}^{N-n-1} \mathbf{1}_{\sigma^{-k} W_{n}} \leq 1, \quad \frac{1}{N-n} \sum_{k=0}^{N-n-1} \mathbf{1}_{\sigma^{-k} W_{n}} \leq \tau \text { on } \Sigma_{N}\left(W_{n}, \tau\right)^{c} .
$$


REMARK: There are two very innovative ideas in this argument. It is somewhat reminiscent of the averaging argument of Theorem 4.1, but there is no averaging over eigenvalues and no appeal to ergodicity (since there is no reason why a weak* limit of $\mu_{\hbar}$ should be an ergodic measure). The first idea is to study the special observables $\hat{P}_{\alpha}$. They are not expressed in the form $O p_{\hbar}(a)$, i.e. in terms of a symbol, and it would be counterproductive to do so. Thus, the argument of (135) takes place entirely on the quantum level and does not make use of any properties of the weak* limit (which are unknown); the semi-classical limit is taken after the inequality is proved. The key innovation is the third step, where the sets $\Sigma_{N}\left(W_{n}, \tau\right)$ are introduced and the sum is broken up into one over $\Sigma_{N}\left(W_{n}, \tau\right)$ and one over its complement. As is evident from $(126), \Sigma_{N}\left(W_{n}, \tau\right)^{c}$ arises naturally as an approximation to the $\tau$-sublevel set of $\frac{1}{N-n} \sum_{k=0}^{N-n-1} \mathbf{1}_{\sigma^{-k} W_{n}}$. But note again that the the decomposition occurs in $\Sigma_{N}$ and is used the on the quantum level. This is very different from using symbols of the $\hat{C}$ and their time averages to to define the corresponding subsets in $S^{*} M$ !

The flaw in the argument is in the third line, where we pretended that $\mu_{\hbar}$ was a positive measure. We recall that $\mu_{\hbar}(\mathcal{C})$ converges to $\mu_{0}(\mathcal{C})$, which is a positive measure. But this is for a fixed $\mathcal{C}$. But it is also close to being a probability measure for cylinder sets of length $\leq \kappa|\log \hbar|$. But (123) is only valid for $N \geq \vartheta|\log \hbar|$. Since $\vartheta>\kappa$, one cannot use them both simultaneously (see $\S 11.10$ for $\kappa, \vartheta$ )

One can attempt to get around this problem using the positivity of the underlying state $\rho_{\hbar}$. Define the function

$$
W_{n}^{N}:=\frac{1}{N-n} \sum_{k=0}^{N-n-1} \mathbf{1}_{\sigma^{-k} W_{n}}: \Sigma \rightarrow \mathbb{R}_{+} .
$$

Then in the third line we rigorously have,

$$
\left|\mu_{\hbar}\left(W_{n}^{N}\right)\right| \leq \rho_{\hbar}\left(\left(\hat{W}_{n}^{N}\right)^{*}\left(\hat{W}_{n}^{N}\right)\right)^{1 / 2}=\left\|\hat{W}_{n}^{N} O p_{\hbar} \psi_{\hbar}\right\|
$$

Here, $\hat{W}_{n}^{N}$ is the quantization as a cylinder set operator. We further denote by $\left.\Sigma_{N} \widehat{\left(W_{n}\right.}, \tau\right)$ the operator equal to the sum of $\hat{\mathcal{C}}$ for $\mathcal{C} \in \Sigma_{N}\left(W_{n}, \tau\right)$. Then

$$
\left.\left.\Sigma_{N} \widehat{\left(W_{n}\right.}, \tau\right)+\Sigma_{N} \widehat{\left(W_{n}\right.}, \tau\right)^{c}=I
$$

So (with $O p_{\hbar}(\chi)$ the energy cutoff),

$$
\begin{aligned}
& \left.\left\|\hat{W}_{n}^{N} O p_{\hbar}(\chi) \psi_{\hbar}\right\| \leq \| \hat{W}_{n}^{N} \Sigma_{N} \widehat{\left(W_{n}\right.}, \tau\right) O p_{\hbar}(\chi) \psi_{\hbar} \| \\
& \quad+\| \hat{W}_{n}^{N} \Sigma_{N} \widehat{\left(W_{n}, \tau\right)^{c} O p_{\hbar}(\chi) \psi_{\hbar} \| .}
\end{aligned}
$$


By $(126)$,

$$
\left\{\begin{array}{l}
\left.\left.\| \hat{W}_{n}^{N} \Sigma_{N} \widehat{\left(W_{n}\right.}, \tau\right) O p_{\hbar}(\chi) \psi_{\hbar}\|\leq\| \Sigma_{N} \widehat{\left(W_{n}\right.}, \tau\right) O p_{\hbar}(\chi) \psi_{\hbar} \| \\
\left.\left.\| \hat{W}_{n}^{N} \Sigma_{N} \widehat{\left(W_{n}\right.}, \tau\right)^{c} O p_{\hbar}(\chi) \psi_{\hbar}\|\leq \tau\| \Sigma_{N} \widehat{\left(W_{n}\right.}, \tau\right)^{c} O p_{\hbar}(\chi) \psi_{\hbar} \| .
\end{array} .\right.
$$

To complete the proof of the final equality, need an estimate of the form,

$$
\left.\| \Sigma_{N} \widehat{\left(W_{n}\right.}, \tau\right) O p_{\hbar}(\chi) \psi_{\hbar} \| \leq 1-\theta
$$

We also need

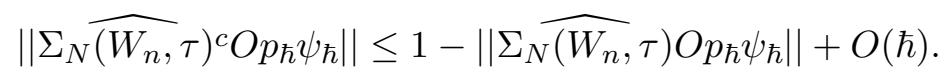

The latter holds formally since $\left.\Sigma_{N} \widehat{\left(W_{n}\right.}, \tau\right), \Sigma_{N} \widehat{\left(W_{n}, \tau\right)^{c}}$ are semi-classically complementary projections. But the time $N \geq \vartheta|\log \hbar|$ may be too large for this semi-classical estimate.

The heuristic arguments are sufficiently convincing to motivate a difficult technical detour in which a certain sub-multiplicativity theorem is used to reduce the $N$ in the argument from $N \geq \vartheta|\log \hbar|$ (above the Ehrenfest time) to $N \leq \kappa|\log \hbar|$ (below the Ehrenfest time). It is the analogue in [A] of the sub-additivity step in $[\mathbf{A N}]$, and its purpose is to allow the argument above to use only cylinder sets of length $\kappa|\log \hbar|$ (or balls of radius $>>\hbar^{1 / 2}$ ) where semi-classical analysis is possible. But one has to make sure that the covering number estimates in Lemma 11.13 and (122) do not break down. One has to choose special covers so that there are not too many elements in the cover of $F$. This step is another crucial innovation in $[\mathbf{A}]$, and in some ways the subtlest one.

11.20.3. ( $\hbar, 1-\theta, n)$-covers. The sub-multiplicativity step requires a new concept and a new parameter $\theta$. Fix $(\hbar, n, \theta)$ and consider a subset $W \subset \Sigma_{n}$.

Definition: Say that $W$ is an $(\hbar, 1-\theta, n)-$ cover of $\Sigma$ if

$$
\left\|\sum_{\mathcal{C} \in W^{c}} \hat{\mathcal{C}}_{\hbar} O p_{\hbar}(\chi) \psi_{\hbar}\right\| \leq \theta .
$$

Here, $W^{c}$ is the complement of $W ; W$ is an $(\hbar, 1-\theta, n)$ cover of $\Sigma$ if in this quantum sense the measure of $W^{c}$ is $<1$ (it is called "small" in $[\mathbf{A}]$ but in the end small means $<1$; See the Remark at the end of $\S 11.20 .4$ ). In other words, the union of the cylinder sets of $W$ might not cover all of $\Sigma$ (think $\left.S^{*} M\right)$, but the mass of the eigenfunctions $\psi_{\hbar}$ outside of the covered part is rather small. In this quantum sense, it is intuitively a union of cylinder sets whose measure is $\geq 1-\theta$. By definition, if $W$ is an $(\hbar, 1-\theta, n)-$ cover, then it is an $\left(\hbar, 1-\theta^{\prime}, n\right)-$ cover for any $\theta^{\prime}>\theta$.

The covering number of such a cover is defined by

$$
N_{\hbar}(n, \theta)=\#\{W: W \text { is a }(\hbar, 1-\theta, n)-\text { cover of } \Sigma\} .
$$


The sub-multiplicative property is

\section{LEMMA 11.16. (sub-multiplicativity)}

$$
N_{\hbar}\left(k n, k \theta\left(1+O\left(n \hbar^{\alpha}\right)\right)\right) \leq N_{\hbar}(n, \theta)^{k} .
$$

We will not attempt to explain this inequality but rather will point out its consequences. One is a lower bound on growth of counting numbers of minimal $(\hbar, 1-\theta, N)$-covers as $N$ grows (Lemma 2.2.6 of $[\mathbf{A}])$ :

LEMma 11.17. Given $\delta>0$, there exists $\vartheta$ so that:

- (i) For $N=\vartheta|\log \hbar|, N_{\hbar}(N, \theta) \geq(1-\theta) e^{N \frac{\Lambda}{2}\left(1-\frac{\delta}{16}\right)}$;

- (ii) For $N=\kappa|\log \hbar|, \kappa \leq \vartheta, N_{\hbar}\left(N, \frac{\kappa}{\vartheta} \theta\right) \geq(1-\theta)^{\frac{\kappa}{\vartheta}} e^{N \frac{\Lambda}{2}\left(1-\frac{\delta}{16}\right)}$;

Estimate (ii) follows from (i) and sub-multiplicativity. The time in (ii) is now below the Ehrenfest time. The sub-multiplicative property then has the following consequence:

LEMma 11.18. (See [A], (2.3.2); compare (131)) Let $\kappa, \vartheta$ have the meanings in $\$ 11.10$. Let $W_{n} \subset \Sigma_{n}$ be a cover of $F$ by $n$-cylinders of minimal cardinality; and let $N=\kappa|\log \hbar|$. Then for any $\theta<1$,

$$
\left\|\sum_{\mathcal{C} \in \Sigma_{N}\left(W_{n}, \tau\right)^{c}} \hat{\mathcal{C}}_{\hbar} O p_{\hbar}(\chi) \psi_{\hbar}\right\| \geq \frac{\kappa}{\vartheta} \theta .
$$

Proof. (Sketch) The inequality goes in the opposite direction from that in the definition of $(\hbar, 1-\theta, N)$-covers in (11.19). Hence the Lemma is equivalent to saying that $\Sigma_{N}(W, \tau)$ is not a $\left(\hbar, 1-\frac{\kappa}{\vartheta} \theta, N\right)$-cover. This follows by comparing the upper bound in Lemma 11.13 with the lower bound in 11.17. If $\Sigma_{N}\left(W_{n}, \tau\right)$ were such a cover, one would have $\frac{\kappa}{\vartheta}|\log (1-\theta)| \geq N \frac{N \Lambda}{2}$, which can only occur for finitely many $N$ if $\theta<1$. The cardinality of $\Sigma_{N}(W, \tau)$ is too small to be $(\hbar, 1-\theta, N)$-cover when $N=\kappa|\log \hbar|$.

The lower bound is a key step in the proof of Theorem 11.5. Intuitively, it gives a lower bound on eigenfunction mass outside the set of orbits which spend a proportion $\tau$ of their time in $W_{n}$. The lower bound on the mass outside $\Sigma_{N}\left(W_{n}, \tau\right)$ eventually prohibits the concentration of the quantum limit measure on $F$.

Using that $\hat{C}_{\hbar} O p_{\hbar}(\chi)$ are roughly orthogonal projectors with orthogonal images for distinct cylinders $\mathcal{C}$, this leads to:

LEMma 11.19. (see $[\mathbf{A}]$, (2.4.5)) Continue with the notation of Lemma 11.18. For $N=\kappa|\log \hbar|$, we have

$$
\left\{\begin{array}{l}
\sum_{\mathcal{C} \in \Sigma_{N}}\left|\mu_{\hbar}(\mathcal{C})\right|=1+O\left(\hbar^{\delta}\right) \\
\sum_{\mathcal{C} \in \Sigma_{N}\left(W_{n}, \tau\right)^{c}}\left|\mu_{\hbar}(\mathcal{C})\right| \geq\left(\frac{\kappa}{\vartheta} \theta\right)^{2}+O\left(\hbar^{\kappa}\right) \\
\Longrightarrow \sum_{\mathcal{C} \in \Sigma_{N}\left(W_{n}, \tau\right)}\left|\mu_{\hbar}(\mathcal{C})\right| \leq 1-\left(\frac{\kappa}{\vartheta} \theta\right)^{2}+O\left(\hbar^{\kappa}\right)
\end{array}\right.
$$


The second inequality of Lemma 11.19 again goes in the opposite direction to the defining inequality (131) of a $(\hbar, 1-\theta, n)$ cover.

11.20.4. Completion of Proof. We now sketch the proof of the Theorem (see $[\mathbf{A}],(2.5 .1)-(2.5 .5))$. It is similar to (124) but circumvents the positivity problem by decreasing $N$ to below the Ehrenfest time.

Proof. (Outline, quoted almost verbatim from $[\mathbf{A}]$ )

As above, the quantum measure $\mu_{\hbar}$ is shift invariant (see $[\mathbf{A}]$, Proposition 1.3.1 (ii).)

We now fix $n$ and let $N=\kappa|\log \hbar|$. The crucial point is that this is less than Ehrenfest time, unlike the choice of $N$ in the first attempt. As above, $W_{n} \subset \Sigma_{n}$ denotes an $(\hbar, 1-\theta, n)$ - cover of minimal cardinality of $F$ by $n$-cylinders. In the following, we use the notation

$$
\mu_{\hbar}\left(W_{n}\right):=\sum_{\mathcal{C} \in W_{n}} \mu_{\hbar}(\mathcal{C}), \quad \mu_{\hbar}\left(\Sigma_{N}\left(W_{n}, \tau\right)\right):=\sum_{\mathcal{C} \in \Sigma_{N}\left(W_{n}, \tau\right)} \mu_{\hbar}(\mathcal{C}) .
$$

We then run through the steps of (124) again;

$$
\begin{aligned}
\left|\mu_{\hbar}\left(W_{n}\right)\right| & =\left|\frac{1}{N-n} \sum_{k=0}^{N-n-1} \mu_{\hbar}\left(\sigma^{-k} W_{n}\right)\right| \\
& =\left|\mu_{\hbar}\left(\frac{1}{N-n} \sum_{k=0}^{N-n-1} \mathbf{1}_{\sigma^{-k} W_{n}}\right)\right| \\
& \leq \sum_{\mathcal{C} \in \Sigma_{N}\left(W_{n}, \tau\right)}\left|\mu_{\hbar}(\mathcal{C})\right|+\tau \sum_{\mathcal{C} \notin \Sigma_{N}\left(W_{n}, \tau\right)}\left|\mu_{\hbar}(\mathcal{C})\right| \\
& \leq \sum_{C \in \Sigma_{N}\left(W_{n}, \tau\right)}\left|\mu_{\hbar}(\mathcal{C})\right|+\tau+O\left(h^{\kappa}\right) \\
& \leq(1-\tau)\left(1-\left(\frac{\kappa}{\vartheta} \theta\right)^{2}\right)+\tau+O\left(h^{\kappa}\right) .
\end{aligned}
$$

Above, Lemma 11.19 is used to circumvent the positivity problem in (124). We also used (again) (126) and the main estimate (123) to bound the $N$-cylinder sets in $\Sigma_{N}\left(W_{n}, \tau\right)$ with $N=\kappa|\log \hbar|$, but with the time $n$ fixed.

Now let $\hbar \rightarrow 0$. Since $W_{n}$ is fixed, the weak convergence $\mu_{\hbar} \rightarrow \mu_{0}$ implies,

$$
\mu_{0}\left(W_{n}\right) \leq(1-\tau)\left(1-\left(\frac{\kappa}{\vartheta} \theta\right)^{2}\right)+\tau .
$$

Since $F \subset W_{n}$, the same estimate applies to $F$. Since it holds for all $\theta<1$, it follows that

$$
\mu_{0}(F) \leq(1-\tau)\left(1-\left(\frac{\kappa}{\vartheta}\right)^{2}\right)+\tau=1-\left(\frac{\kappa}{\vartheta}\right)^{2}(1-\tau)<1 .
$$

This completes the proof that supp $\nu_{0} \neq F$. QED 
To obtain the measure statement of Theorem 11.5, let

$$
I_{\delta}=\left\{x \in S^{*} M: h_{g}\left(\mu_{0}^{x}\right) \leq \frac{\Lambda}{2}(1-\delta)\right\} .
$$

It follows from the Shannon-McMillan theorem that for all $\alpha>0$, there exists $I_{\delta}^{\alpha} \subset I_{\delta}$ with $\mu_{0}\left(I_{\delta} \backslash I_{\delta}^{\alpha}\right) \leq \alpha$ such that $I_{\delta}^{\alpha}$ can be covered by $e^{n\left(\frac{\Lambda}{2}(1-\delta+\alpha)\right.}$ n-cylinders for sufficiently large $n$. It follows from (137) that

$$
\mu_{0}\left(I_{\delta}^{\alpha}\right) \leq(1-\tau(\delta-\alpha))\left(1-\left(\frac{\kappa}{\vartheta(\delta-\alpha)}\right)_{+}^{2}\right)+\tau(\delta-\alpha) .
$$

Let $\alpha \rightarrow 0$ and one has,

$$
\nu_{0}\left(S^{*} M \backslash I_{\delta}\right) \geq(1-\tau(\delta))\left(\frac{\kappa}{\vartheta(\delta)}\right)^{2} .
$$

11.21. $F=\gamma$. To help digest the proof, let us run through the proof in the simplest case where $F$ is a single closed hyperbolic geodesic $\gamma$ and $M$ is a compact hyperbolic manifold of constant curvature -1 . We would like to show that $\nu_{0} \neq \mu_{\gamma}$, or equivalently that $\nu_{0}(\gamma)<1$. What we need to show is that, if $\nu_{0}(F)=1$, then it requires a lot of cylinder sets to cover $F$. Hence we try to get a contradiction from the fact that only a few cylinder sets are needed to cover $\gamma$. In fact, for each $n, \gamma$ may be covered by one $n$-cylinder

$$
W_{n}^{0}(\gamma)=\left\{\left[\alpha_{0}, \ldots, \alpha_{0}, \ldots, \alpha_{n}\right]\right\},
$$

namely the one specified by the indices of the cells $P_{\alpha}$ that $\gamma$ passes through starting from some fixed $x_{0} \in \gamma$. To obtain a tube around the orbit, we take the union $W_{n}(\gamma):=\bigcup_{j=0}^{n-1} g^{-j} W_{n}^{0}(\gamma)$. We could use the two-sided symbols, going symmetrically forward and backward in time to obtain a transverse ball of radius $e^{-n / 2}$.

First, let us try a naive heuristic argument. The main estimate (113) gives

$$
\left|\mu_{\hbar}\left(W_{n}(\gamma)\right)\right| \leq C h^{-d / 2} e^{-n d / 2}(1+O(\epsilon))^{n}, \quad \text { uniformly for } n \leq K|\log \hbar|,
$$

for any $K>0$. It is now tempting to put $n=(1+\epsilon)|\log \hbar|$ with $\epsilon>0$ to obtain $\left|\mu_{\hbar}\left(W_{n(\hbar, \theta)}\right)\right| \leq \hbar^{\theta}$. But we cannot take the semi-classical limit of this estimate. If we did so formally (not rigorously), it would seem to imply that $\nu_{0}(\gamma)=0$, which is stronger than the rigorous result $\nu_{0}(\gamma)<1$ and possibly false in some examples (if weak scarring in fact occurs).

The problem is that the classical set $W_{n(\hbar, \theta)}$ is a kind of $\hbar$-dependent Bowen ball or tube around $\gamma$ of radius $e^{-\frac{1}{2} n(\hbar, \theta)}=\hbar^{1 / 2+\theta}$. The uncertainty principle prohibits microlocalization to tubes of radius $<h^{\frac{1}{2}}$ and (as discussed in $§ 5)$, it is difficult even to work with neighborhoods smaller than $h^{\frac{1}{2}-\delta}$ or at best $h^{\frac{1}{2}}|\log \hbar|$. In particular, we do not have control over the weak limits of $\left|\mu_{\hbar}\left(W_{n(\hbar, \theta)}\right)\right|$, and there is no obvious reason why it should 
have semi-classical asymptotics. It is not a positive measure on this length scale, so we do not know if it implies $\nu_{0}(\gamma)=0$. On the other hand, if we decrease $n$ below the Ehrenfest time, so that the matrix element has a classical limit, then the bound is trivial.

The strategy of $[\mathbf{A}]$ is to use the sub-multiplicative estimate for $(\hbar, 1-$ $\theta, N)$-covers of $\gamma$ of minimal cardinality to decrease the length $N$ below the Ehrenfest time (see Lemma 11.17). We first consider the cover $W_{n}:=W_{n}(\gamma)$, which is a minimal cover of $\gamma$ by cylinders of length $n$. We then form $\Sigma_{N}\left(W_{n}, \tau\right)$. At least intuitively, $W_{n}$ corresponds to a Bowen ball of radius $e^{-n / 2}$, i.e. a transverse cube of this radius in the stable and unstable directions. The cylinders of $\Sigma_{N}\left(W_{n}, \tau\right)$ satisfy $\sigma^{j}(\mathcal{C}) \cap W_{n}=\mathcal{C}$ for a proportion $\tau$ of $0 \leq j \leq N$. This carves out a very porous set in the stable/unstable cross section, suggesting why the lower bound in Lemma 11.19 should be true.

It would be interesting to connect the argument of Theorem 11.5 in this case to the problem in $\S 5$ (see $\S 5.1$ ) of estimating the eigenfunction mass inside a shrinking tubular neighborhood of $\gamma$, i.e. to give an upper bound for the mass $\left(O p_{\hbar}\left(\chi_{1}^{\delta}\right) \varphi_{\mu}, \varphi_{\mu}\right)$ in the shrinking tube around $\gamma$ or (better) the mass $\left(O p_{\hbar}\left(\chi_{2}^{\delta}\right) \varphi_{\mu}, \varphi_{\mu}\right)$ in a shrinking phase space tube. However, the methods of $[\mathbf{A}]$ apply equally to the cat map setting and in that case, there are eigenfunctions whose mass in shrinking tubes around a hyperbolic fixed point have the same order of magnitude as in the integrable case up to a factor of $1 / 2[\mathbf{F N B}]$. So unless one can use a property of $\Delta$-eigenfunctions which is not shared by quantum cat map eigenfunctions, one cannot expect to prove a sharper upper bound for eigenfunction mass in shrinking tubes around hyperbolic orbits in the Anosov case than one has in the integrable case.

11.22. Some ideas on the main estimate. The main estimate is independently useful. For instance, it has been used to obtain bounds on decay rates on quantum scattering $[\mathbf{N Z}]$. In this section, we sketch some ideas of the proof. We do not go so far as to explain a key point, that the estimate is valid for times $t \leq \kappa|\log \hbar|$ for any $\kappa$.

The goal is to estimate the norm of the operators $P_{\alpha^{\prime}}^{*} U^{n} P_{\alpha} \mathrm{Op}\left(\chi^{(n)}\right)$ in terms of $\hbar$ and $n$. Since $U(n)$ is unitary it suffices to estimate the norm of the cylinder set operators

$$
K_{n}:=\hat{P}_{\alpha_{n}} U \hat{P}_{\alpha_{n}-1} \cdots U \hat{P}_{\alpha_{0}} O p(\chi)
$$

Let $K_{n}(z, w)$ be the Schwartz kernel of this operator. By the Schur inequality, the operator norm is bounded above by

$$
\sup _{z} \int_{M}\left|K_{n}(z, w)\right| d V(w) \text {. }
$$

The inequality only applies when $K_{n}(z, w) \in L^{1}(M)$, but by harmless smoothing approximation we may assume this.

We may get an intuitive idea of the exponential decay of the norm of the cylinder set operators as follows. The wave group (or the semi-classical 
Schrödinger propagator) $U^{t}$ on a manifold without conjugate points can be lifted to the universal cover (see (25)) where it has a global Hadamard parametrix (17). This parametrix shows that the wave kernel on the universal cover is closely related to the spherical means operator $L_{t} f(x)=$ $\int_{S_{x}^{*} M} f\left(g^{t}(x, \omega) d \mu_{x}(\omega)\right.$, where $d \mu_{x}$ is the Euclidean surface measure on $S_{x}^{*} M$ induced by the metric. Both operators have the same wave front relation, and indeed the wave front set of $U(t, \cdot, y)$ is precisely the set of (co-)normal directions to the distance sphere $S_{t}(y)$ centered at $y$. The operator relating $U^{t}$ and $L_{t}$ is a $d$ th order (pseudo-differential) operator in $(t, x)$, and in the Fourier transformed picture in $[\mathbf{A}]$ it gives rise to the factor $h^{-d / 2}$. As time evolves, the surface volume of the distance sphere in the universal cover grows exponentially. Since $U^{t}$ is unitary, its amplitude must decay exponentially. In fact this is evident from (17) since $\Theta(x, y)$ grows exponentially in the distance from $x$ to $y$, so $\Theta^{-1 / 2}(x, y)$ decays exponentially.

The cylinder set operators $\hat{\mathcal{C}}_{\hbar}=\hat{P}_{\alpha_{n}}(n) \cdots \hat{P}_{\alpha_{1}}(1) \hat{P}_{\alpha_{0}}(0)$ are given by a sequence of alternating applications of the propagator $U$ and the cutting off operators $P_{\alpha_{j}}$ (which are multiplications). At least heuristically, in estimating the Schur integral for $K_{n}(x, y)$, one is first cutting off to a piece of the distance of the distance sphere $S_{1}(y)$ of radius one centered at $y$. Then one applies $U$ and this piece expands to $S_{2}(y)$. Then one cuts it off with $P_{\alpha_{2}}$. As this picture evolves, one ends up after $n$ iterates with a piece of the distance sphere $S_{n}(y)$ of radius $n$ centered at $y$ which is has the same size as the initial piece. But the spherical means and wave group are normalized by dividing by the volume, so the contribution to the Schur integral is exponentially decaying at the rate $e^{-n}$.

\subsection{Clarifications to $[\mathbf{A}]$.}

(1) The $\theta$ on p. 447 is a new parameter $<1$ which is implicitly defined above (2.0.1).

(2) The $\theta$ in (2.3.2) is also implicitly defined by this inequality. $W_{n_{1}}$ is not assumed to be a $\theta$ cover.

(3) In (2.5.1)-(2.5.5), the $\Sigma$ in $\sigma^{-k} \Sigma\left(W_{n_{1}}\right)$ should be removed.

(4) As mentioned above, the notation $\mu_{h}\left(\Sigma_{N}\left(W_{n}, \tau\right)\right)$ is not quite defined but means $\mu_{h}\left(\bigcup_{\left.\mathcal{C} \in \Sigma_{N}\left(W_{n}, \tau\right)\right)} \mathcal{C}\right)$.

\section{Applications to nodal hypersurfaces of eigenfunctions}

This survey has been devoted to the asymptotics of matrix elements $\left\langle A \varphi_{j}, \varphi_{j}\right\rangle$. In the final section, we address the question, "what applications does the study of these matrix elements have?"

In automorphic forms, the matrix elements are related to L-functions, Rankin-Selberg zeta functions, etc. So there is ample motivation for their study in the arithmetic case. Such applications lie outside the scope of this survey, so we refer the reader to Sarnak's expository articles [Sar2, Sar3] for 
background on and references to the large literature in arithmetic quantum chaos.

Here we are concerned with applications to the classical and geometric analysis of eigenfunctions on general Riemannian manifolds. We present an application of quantum ergodicity to the study of nodal (zero) sets of eigenfunctions on real analytic $(M, g)$ with ergodic geodesic flow. It is not implausible that one could use related methods to count critical points of eigenfunctions in the ergodic case.

The nodal hypersurface of $\varphi_{j}$ is the zero set $\mathcal{N}_{j}=\left\{x \in M: \varphi_{j}(x)=0\right\}$. We are interested in its distribution as $\lambda_{j} \rightarrow \infty$. We study the distribution through the integrals

$$
\int_{\mathcal{N}_{j}} f d S
$$

of $f$ over the nodal line with respect to the natural surface measure. If $f=1_{U}$ is the characteristic (indicator) function of an open set $U \subset M$, then

$$
\int_{\mathcal{N}_{j}} 1_{U} d s=\mathcal{H}^{n-1}\left(U \cap \mathcal{N}_{j}\right)
$$

is $(n-1)$ dimensional Hausdorff measure of the part of $\mathcal{N}_{j}$ which intersects $U$. When $U=M$ the integral (142) gives the total surface volume of the nodal set. The principal result on volumes is due to Donnelly-Fefferman [DF], proving a conjecture of S. T. Yau [Y1]:

TheOREM 12.1. [DF] (see also $[\mathbf{L i n}]$ ) Let $(M, g)$ be a compact real analytic Riemannian manifold, with or without boundary. Then there exist $c_{1}, C_{2}$ depending only on $(M, g)$ such that

$$
c_{1} \lambda \leq \mathcal{H}^{m-1}\left(Z_{\varphi_{\lambda}}\right) \leq C_{2} \lambda, \quad\left(\Delta \varphi_{\lambda}=\lambda^{2} \varphi_{\lambda} ; c_{1}, C_{2}>0\right) .
$$

The question is whether one can obtain asymptotics results by imposing a global dynamical condition on the geodesic flow.

The hypothesis of real analyticity is crucial: The analysis is based on the analytic continuation of eigenfunctions to the complexification $M_{\mathbb{C}}$ of $M$. The articles $[\mathbf{D F}, \mathbf{L i n}]$ also used analytic continuation to study volumes of nodal hypersurfaces. It is the role of ergodicity in the complex setting that gives strong equidistribution results.

12.1. Complexification of $(M, g)$ and Grauert tubes. By a theorem of Bruhat and Whitney, a real analytic manifold $M$ always possesses a unique complexification $M_{\mathbb{C}}$, an open complex manifold in which $M$ embeds $\iota: M \rightarrow M_{\mathbb{C}}$ as a totally real submanifold. Examples are real algebraic subvarieties of $\mathbb{R}^{n}$ which complexify to complex algebraic subvarieties of $\mathbb{C}^{n}$. Other simple examples include the complexification of a quotient such as $M=\mathbb{R}^{m} / \mathbb{Z}^{m}$ to $M_{\mathbb{C}}=\mathbb{C}^{m} / \mathbb{Z}^{m}$. The complexification of hyperbolic space can be defined using the hyperboloid model $\mathbb{H}^{n}=\left\{x_{1}^{2}+\cdots x_{n}^{2}-x_{n+1}^{2}=\right.$ $\left.-1, \quad x_{n}>0\right\}$. Then, $\mathbb{H}_{\mathbb{C}}^{n}=\left\{\left(z_{1}, \ldots, z_{n+1}\right) \in \mathbb{C}^{n+1}: z_{1}^{2}+\cdots z_{n}^{2}-z_{n+1}^{2}=-1\right\}$. 
The Riemannian metric determines a special kind of plurisubharmonic exhaustion function on $M_{\mathbb{C}}[\mathbf{G S 1}, \mathbf{G S 2}$, LemS1], namely the Grauert tube radius $\sqrt{\rho}=\sqrt{\rho}_{g}$ on $M_{\mathbb{C}}$, defined as the unique solution of the MongeAmpère equation

$$
(\partial \bar{\partial} \sqrt{\rho})^{m}=\delta_{M_{\mathbb{R}}, d V_{g}}, \quad \iota^{*}(i \partial \bar{\partial} \rho)=g .
$$

Here, $\delta_{M_{\mathbb{R}}, d V_{g}}$ is the delta-function on the real $M$ with respect to the volume form $d V_{g}$, i.e. $f \rightarrow \int_{M} f d V_{g}$. It is shown in [GS1] that $\sqrt{\rho}(\zeta)=i \sqrt{r^{2}(\zeta, \bar{\zeta})}$ where $r^{2}(x, y)$ is the squared distance function in a neighborhood of the diagonal in $M \times M$.

The Grauert tubes $M_{\tau}$ is defined by

$$
M_{\tau}=\left\{\zeta \in M_{\mathbb{C}}: \sqrt{\rho}(\zeta) \leq \tau\right\}
$$

The maximal value of $\tau_{0}$ for which $\sqrt{\rho}$ is well defined is known as the Grauert tube radius. For $\tau \leq \tau_{0}, M_{\tau}$ is a strictly pseudo-convex domain in $M_{\mathbb{C}}$. Using the complexified exponential map $(x, \xi) \rightarrow e_{x} p_{x} i \xi$ one may identify $M_{\tau}$ with the co-ball bundle $B_{\tau}^{*} M$ to $M_{\tau}$. Under this identification, $\sqrt{\rho}$ corresponds to $|\xi|_{g}$. The one-complex dimensional null foliation of $\partial \bar{\partial} \sqrt{\rho}$, known as the "Monge-Ampère" or Riemann foliation, are the complex curves $t+i \tau \rightarrow \tau \dot{\gamma}(t)$, where $\gamma$ is a geodesic. The geometric Grauert tube radius is the maximal radius for which the exponential map has a holomorphic extension and defines a diffeomorphism.

12.2. Analytic Continuation of eigenfunctions. The eigenfunctions are real analytic and therefore possess analytic continuations to some Grauert tube $M_{\epsilon}$ independent of the eigenvalue. To study analytic continuation of eigenfunctions, it is useful to relate them to analytic continuation of the wave group at imaginary time, $U(i \tau, x, y)=e^{-\tau \sqrt{\Delta}}(x, y)$. This is the Poisson operator of $(M, g)$. We denote its analytic continuation in the $x$ variable to $M_{\epsilon}$ by $U_{\mathbb{C}}(i \tau, \zeta, y)$. In terms of the eigenfunction expansion, one has

$$
U(i \tau, \zeta, y)=\sum_{j=0}^{\infty} e^{-\tau \lambda_{j}} \varphi_{j}^{\mathbb{C}}(\zeta) \varphi_{j}(y), \quad(\zeta, y) \in M_{\epsilon} \times M
$$

To the author's knowledge, the largest $\epsilon$ has not been determined at this time. In particular, it is not known if the radius of analytic continuation of the wave kernel and of the eigenfunctions is the same as the geometrically defined Grauert tube radius.

Since

$$
U_{\mathbb{C}}(i \tau) \varphi_{\lambda}=e^{-\tau \lambda} \varphi_{\lambda}^{\mathbb{C}}
$$

the analytic continuability of the Poisson operator to $M_{\tau}$ implies that every eigenfunction analytically continues to the same Grauert tube. It follows 
that the analytic continuation operator to $M_{\tau}$ is given by

$$
A_{\mathbb{C}}(\tau)=U_{\mathbb{C}}(i \tau) \circ e^{\tau \sqrt{\Delta}}
$$

Thus, a function $f \in C^{\infty}(M)$ has a holomorphic extension to the closed tube $\sqrt{\rho}(\zeta) \leq \tau$ if and only if $f \in \operatorname{Dom}\left(e^{\tau \sqrt{\Delta}}\right)$, where $e^{\tau \sqrt{\Delta}}$ is the backwards "heat operator" generated by $\sqrt{\Delta}$ (rather than $\Delta$ ).

Let us consider examples of holomorphic continuations of eigenfunctions:

- On the flat torus $\mathbb{R}^{m} / \mathbb{Z}^{m}$, the real eigenfunctions are $\cos \langle k, x\rangle$, $\sin \langle k, x\rangle$ with $k \in 2 \pi \mathbb{Z}^{m}$. The complexified torus is $\mathbb{C}^{m} / \mathbb{Z}^{m}$ and the complexified eigenfunctions are $\cos \langle k, \zeta\rangle, \sin \langle k, \zeta\rangle$ with $\zeta=x+i \xi$.

- On the unit sphere $S^{m}$, eigenfunctions are restrictions of homogeneous harmonic functions on $\mathbb{R}^{m+1}$. The latter extend holomorphically to holomorphic harmonic polynomials on $\mathbb{C}^{m+1}$ and restrict to holomorphic function on $S_{\mathbb{C}}^{m}$.

- On $\mathbf{H}^{m}$, one may use the hyperbolic plane waves $e^{(i \lambda+1)\langle z, b\rangle}$, where $\langle z, b\rangle$ is the (signed) hyperbolic distance of the horocycle passing through $z$ and $b$ to 0 . They may be holomorphically extended to the maximal tube of radius $\pi / 4$. See e.g. [BR].

- On compact hyperbolic quotients $\mathbf{H}^{m} / \Gamma$, eigenfunctions can be then represented by Helgason's generalized Poisson integral formula,

$$
\varphi_{\lambda}(z)=\int_{B} e^{(i \lambda+1)\langle z, b\rangle} d T_{\lambda}(b) .
$$

To analytically continue $\varphi_{\lambda}$ it suffices to analytically continue $\langle z, b\rangle$. Writing the latter as $\langle\zeta, b\rangle$, we have:

$$
\varphi_{\lambda}^{\mathbb{C}}(\zeta)=\int_{B} e^{(i \lambda+1)\langle\zeta, b\rangle} d T_{\lambda}(b) .
$$

- In the case of 1D Schrödinger operators, several authors have studied analytic continuations of eigenfunctions and their complex zeros, see e.g. [Hez, EGS].

12.3. Maximal plurisubharmonic functions and growth of $\varphi_{\lambda}^{\mathbb{C}}$. In the case of domains $\Omega \subset \mathbb{C}^{m}$, the maximal PSH (pluri-subharmonic) function (or pluri-complex Green's function) relative to a subset $E \subset \Omega$ is defined by

$$
V_{E}(\zeta)=\sup \left\{u(z): u \in P S H(\Omega),\left.u\right|_{E} \leq 0,\left.u\right|_{\partial \Omega} \leq 1\right\}
$$

An alternative definition due to Siciak takes the supremum only with respect to polynomials $p$. We denote by $\mathcal{P}^{N}$ the space of all complex analytic polynomials of degree $N$ and put $\mathcal{P}_{K}^{N}=\left\{p \in \mathcal{P}^{N}:\|p\|_{K} \leq 1,\|p\|_{\Omega} \leq e\right\}$. Then define

$$
\log \Phi_{E}^{N}(\zeta)=\sup \left\{\frac{1}{N} \log \left|p_{N}(\zeta)\right|: p \in \mathcal{P}_{E}^{N}\right\}, \quad \log \Phi_{E}=\limsup _{N \rightarrow \infty} \log \Phi_{E}^{N} .
$$


Here, $\|f\|_{K}=\sup _{z \in K}|f(z)|$. Siciak proved that $\log \Phi_{E}=V_{E}$.

On a real analytic Riemannian manifold, the natural analogue of $\mathcal{P}^{N}$ is the space

$$
\mathcal{H}^{\lambda}=\left\{p=\sum_{j: \lambda_{j} \leq \lambda} a_{j} \varphi_{\lambda_{j}}, a_{1}, \ldots, a_{N(\lambda)} \in \mathbb{R}\right\}
$$

spanned by eigenfunctions with frequencies $\leq \lambda$. Rather than using the sup norm, it is convenient to work with $L^{2}$ based norms than sup norms, and so we define

$$
\mathcal{H}_{M}^{\lambda}=\left\{p=\sum_{j: \lambda_{j} \leq \lambda} a_{j} \varphi_{\lambda_{j}}, \quad\|p\|_{L^{2}(M)}=\sum_{j=1}^{N(\lambda)}\left|a_{j}\right|^{2}=1\right\} .
$$

We define the $\lambda$-Siciak approximate extremal function by

$$
\Phi_{M}^{\lambda}(z)=\sup \left\{|\psi(z)|^{1 / \lambda}: \psi \in \mathcal{H}_{\lambda} ;\|\psi\|_{M} \leqslant 1\right\},
$$

and the extremal function by

$$
\Phi_{M}(z)=\sup _{\lambda} \Phi_{M}^{\lambda}(z) .
$$

It is not hard to show that

$$
\Phi_{M}(z)=\lim _{\lambda \rightarrow \infty} \frac{1}{\lambda} \log \Pi_{[0, \lambda}(\zeta, \bar{\zeta})=\sqrt{\rho} .
$$

12.4. Nodal hypersurfaces in the case of ergodic geodesic flow. The complex nodal hypersurface of an eigenfunction is defined by

$$
Z_{\varphi_{\lambda}^{\mathbb{C}}}=\left\{\zeta \in B_{\epsilon_{0}}^{*} M: \varphi_{\lambda}^{\mathbb{C}}(\zeta)=0\right\}
$$

There exists a natural current of integration over the nodal hypersurface in any ball bundle $B_{\epsilon}^{*} M$ with $\epsilon<\epsilon_{0}$, given by

$$
\left\langle\left[Z_{\varphi_{\lambda}^{\mathbb{C}}}^{\mathbb{C}}\right], \varphi\right\rangle=\frac{i}{2 \pi} \int_{B_{\epsilon}^{*} M} \partial \bar{\partial} \log \left|\varphi_{\lambda}^{\mathbb{C}}\right|^{2} \wedge \varphi=\int_{Z_{\varphi_{\lambda}^{\mathbb{C}}}} \varphi, \quad \varphi \in \mathcal{D}^{(m-1, m-1)}\left(B_{\epsilon}^{*} M\right) .
$$

In the second equality we used the Poincaré-Lelong formula. The notation $\mathcal{D}^{(m-1, m-1)}\left(B_{\epsilon}^{*} M\right)$ stands for smooth test $(m-1, m-1)$-forms with support in $B_{\epsilon}^{*} M$.

The nodal hypersurface $Z_{\varphi_{\lambda}^{\mathbb{C}}}$ also carries a natural volume form $\left|Z_{\varphi_{\lambda}^{\mathbb{C}}}\right|$ as a complex hypersurface in a Kähler manifold. By Wirtinger's formula, it equals the restriction of $\frac{\omega_{g}^{m-1}}{(m-1) !}$ to $Z_{\varphi_{\lambda}^{\mathbb{C}}}$.

THEOREM 12.2. [Z5] Let $(M, g)$ be real analytic, and let $\left\{\varphi_{j_{k}}\right\}$ denote a quantum ergodic sequence of eigenfunctions of its Laplacian $\Delta$. Let $\left(B_{\epsilon_{0}}^{*} M, J\right)$ be the maximal Grauert tube around $M$ with complex structure $J_{g}$ adapted to $g$. Let $\epsilon<\epsilon_{0}$. Then:

$$
\frac{1}{\lambda_{j_{k}}}\left[Z_{\varphi_{j_{k}}^{\mathbb{C}}}\right] \rightarrow \frac{i}{\pi} \partial \bar{\partial} \sqrt{\rho} \text { weakly in } \mathcal{D}^{\prime}(1,1)\left(B_{\epsilon}^{*} M\right)
$$


in the sense that, for any continuous test form $\psi \in \mathcal{D}^{(m-1, m-1)}\left(B_{\epsilon}^{*} M\right)$, we have

$$
\frac{1}{\lambda_{j_{k}}} \int_{Z_{\varphi_{j_{k}}^{\mathbb{C}}}} \psi \rightarrow \frac{i}{\pi} \int_{B_{\epsilon}^{*} M} \psi \wedge \partial \bar{\partial} \sqrt{\rho}
$$

Equivalently, for any $\varphi \in C\left(B_{\epsilon}^{*} M\right)$,

$$
\frac{1}{\lambda_{j_{k}}} \int_{Z_{\varphi_{j_{k}}^{\mathbb{C}}}} \varphi \frac{\omega_{g}^{m-1}}{(m-1) !} \rightarrow \frac{i}{\pi} \int_{B_{\epsilon}^{*} M} \varphi \partial \bar{\partial} \sqrt{\rho} \wedge \frac{\omega_{g}^{m-1}}{(m-1) !} .
$$

Corollary 12.3. Let $(M, g)$ be a real analytic with ergodic geodesic flow. Let $\left\{\varphi_{j_{k}}\right\}$ denote a full density ergodic sequence. Then for all $\epsilon<\epsilon_{0}$,

$$
\frac{1}{\lambda_{j_{k}}}\left[Z_{\varphi_{j_{k}}^{\mathbb{C}}}\right] \rightarrow \frac{i}{\pi} \partial \bar{\partial} \sqrt{\rho} \text {, weakly in } \mathcal{D}^{\prime(1,1)}\left(B_{\epsilon}^{*} M\right) \text {. }
$$

REMARK: The limit form is singular with respect to the Kähler form $i \partial \bar{\partial} \rho$ on $M_{\mathbb{C}}$ adapted to the metric. Its highest exterior power is the delta function on the zero section (i.e. the real domain $M$ ).

In outline the steps in the proof are;

(1) By the Poincaré-Lelong formula, $\left[Z_{\varphi_{\lambda}^{\mathbb{C}}}\right]=i \partial \bar{\partial} \log \left|\varphi_{\lambda}^{\mathbb{C}}\right|$. This reduces the theorem to determining the limit of $\frac{1}{\lambda} \log \left|\varphi_{\lambda}^{\mathbb{C}}\right|$.

(2) $\left|\varphi_{\lambda}^{\mathbb{C}}\right|^{2}$, when properly $L^{2}$ normalized on each $\partial M_{\tau}$ is a quantum ergodic sequence on $\partial M_{\tau}$.

(3) The ergodic property of complexified eigenfunctions implies that the $L^{2}$ norm of $\left|\varphi_{\lambda}^{\mathbb{C}}\right|^{2}$ on $\partial M_{\tau}$ is asymtotically $\sqrt{\rho}$. Thus, normalized $\log$ moduli of ergodic eigenfunctions are asympotically maximal PSH functions. They are $\lambda$-Siciak extremal functions.

(4) $\frac{1}{\lambda} \log \frac{\left|\varphi_{\lambda}^{\mathbb{C}}(\zeta)\right|^{2}}{\|\left.\varphi_{\lambda}^{\mathbb{C}}\right|_{L^{2}\left(\partial M_{\tau}\right.} ^{2}} \rightarrow 0$ for an ergodic sequence of eigenfunctions. Hence,

(5) $\frac{i}{\lambda} \partial \bar{\partial} \log \left|\varphi_{\lambda}^{\mathbb{C}}\right| \rightarrow i \partial \bar{\partial} \sqrt{\rho}$,

concluding the proof.

REMARK: We expect a similar result for nodal lines of Dirichlet or Neumann eigenfunctions of a piecewise smooth domain $\Omega \subset \mathbb{R}^{2}$ with ergodic billiards. We also expect similar results for nodal lines of such domains which touch the boundary (like the angular "spokes" of eigenfunctions of the disc). In $[\mathbf{T Z 1}$ ], we proved that the number of such nodal lines is bounded above by $C_{\Omega} \lambda$. This is rather like the Donnelly-Fefferman upper bound. However the example of the disc shows that there is no lower bound above zero. That is, there are sequences of eigenfunctions (with low angular momentum) with a uniformly bounded number of nodal lines touching the boundary. We believe that ergodicity is a mechanism producing lower bounds on numbers of zeros and critical points. It causes eigenfunctions to oscillate uniformly 
in all directions and hence to produce a lot of zeros and critical points. We expect this to be one of the future roles for ergodicity of eigenfunctions.

REMARK: Precursors to Theorem 12.2 giving limit distributions of zero sets for holomorphic eigen-sections of Kähler quantizations of ergodic symplectic maps on Kähler manifolds can be found in [NV2, SZ]. In that setting, the zeros become uniformly distributed with respect to the Kähler form. In the Riemannian setting, they become uniformly distributed with respect to a singular $(1,1)$ current relative to the Kähler form $\partial \bar{\partial} \rho$ on $M_{\mathbb{C}}$.

\section{References}

[A] N. Anantharaman, Entropy and the localization of eigenfunctions, Ann. of Math. (2) 168 (2008), no. 2, 435-475.

[A2] N. Anantharaman, Eigenfunctions of the Laplacian on negatively curved manifolds: a semi-classical approach, Lecture Notes (online at http://www.math.polytechnique.fr/ nalini/).

[ANK] N. Anantharaman, H. Koch, and S. Nonnenmacher, Entropy of eigenfunctions, arXiv:0704.1564.

[AN] N. Anantharaman and S. Nonnenmacher, Half-delocalization of eigenfunctions for the Laplacian on an Anosov manifold, Annales Inst. Fourier 57, number 6 (2007), 2465-2523 (arXiv:math-ph/0610019).

[AN2] N. Anantharaman and S. Nonnenmacher, Vibrations chaotiques et grosses balafres. Gaz. Math. No. 119 (2009), 16-32. English Version (different): Chaotic vibrations and strong scars.

[AZ] N. Anantharaman and S. Zelditch, Patterson-Sullivan distributions and quantum ergodicity. Ann. Henri Poincar 8 (2007), no. 2, 361-426.

[AS] R. Aurich and F. Steiner, Statistical properties of highly excited quantum eigenstates of a strongly chaotic system. Phys. D 64 (1993), no. 1-3, 185-214.

[B.B] V. M. Babic and V. S. Buldyrev, Short-Wavelength Diffraction Theory, Springer Series on Wave Phenomena 4, Springer-Verlag, New York (1991).

[BSS] A. Backer, R. Schubert, and P. Stifter, On the number of bouncing ball modes in billiards. J. Phys. A 30 (1997), no. 19, 6783-6795.

[Bar] A. Barnett, Asymptotic rate of quantum ergodicity in chaotic Euclidean billiards, Comm. Pure Appl. Math. 59 (2006), no. 10, 1457-1488.

[Be] P. Bérard, On the wave equation without conjugate points, Math. Zeit. 155 (1977), 249-276.

[BR] J. Bernstein and A. Reznikov, Analytic continuation of representations and estimates of automorphic forms. Ann. of Math. (2) 150 (1999), no. 1, 329-352.

[Ber] M. Berry, Regular and irregular semiclassical wavefunctions, J. Phys. A 10 (1977), 2083-2091.

[BL] J. Bourgain and E. Lindenstrauss, Entropy of quantum limits. Comm. Math. Phys. 233 (2003), no. 1, 153-171.

[BouR] A. Bouzouina and D. Robert, Uniform semiclassical estimates for the propagation of quantum observables. Duke Math. J. 111 (2002), no. 2, 223-252.

$[\mathrm{Bu}] \quad$ N. Burq, Quantum ergodicity of boundary values of eigenfunctions: A control theory approach, Canad. Math. Bull. 48 (2005), no. 1, 3-15 (math.AP/0301349).

[BZ] N. Burq and M. Zworski, Geometric control in the presence of a black box. J. Amer. Math. Soc. 17 (2004), no. 2, 443-471. 
[Chr] H. Christianson, Semiclassical non-concentration near hyperbolic orbits. J. Funct. Anal. 246 (2007), no. 2, 145-195.

[CV] Y. Colin de Verdière, Ergodicité et fonctions propres du Laplacien, Comm. Math. Phys. 102 (1985), 497-502.

[CV2] Y. Colin de Verdière, Quasi-modes sur les varietes Riemanniennes compactes, Invent. Math. 43 (1977), 15-52.

[CV3] Y. Colin de Verdière, Semi-classical measures and entropy [after Nalini Anantharaman and Stéphane Nonnenmacher]. Séminaire Bourbaki. Vol. 2006/2007. Astérisque No. 317 (2008), Exp. No. 978, ix, 393-414.

[CVP] Y. Colin de Verdière and B. Parisse, Équilibre instable en régime semi-classique. I. Concentration microlocale, Comm. in PDE 19 (1994), no. 9-10, 1535-1563.

[CR] M. Combescure and D. Robert, Semiclassical spreading of quantum wave packets and applications near unstable fixed points of the classical flow, Asymptot. Anal. 14 (1997), 377-404.

[DSj] M. Dimassi and J. Sjöstrand, Spectral asymptotics in the semi-classical limit. London Mathematical Society Lecture Note Series, 268. Cambridge University Press, Cambridge (1999).

[DF] H. Donnelly and C. Fefferman, Nodal sets of eigenfunctions on Riemannian manifolds, Invent. Math. 93 (1988), 161-183.

[Dui] J. J. Duistermaat, Oscillatory integrals, Lagrange immersions and unfolding of singularities. Comm. Pure Appl. Math. 27 (1974), 207-281.

[D.G] J.J.Duistermaat and V.Guillemin, The spectrum of positive elliptic operators and periodic bicharacteristics, Inv.Math. 24 (1975), 39-80.

[EGS] A. Eremenko, A. Gabrielov, and B. Shapiro, High energy eigenfunctions of one-dimensional Schrödinger operators with polynomial potentials. Comput. Methods Funct. Theory 8 (2008), no. 1-2, 513-529.

[EZ] L. Evans and M. Zworski, Introduction to semiclassical analysis, UC Berkeley lecture notes (2006). http://math.berkeley.edu/ zworski.

[FN] F. Faure and S. Nonnenmacher, On the maximal scarring for quantum cat map eigenstates. Comm. Math. Phys. 245 (2004), no. 1, 201-214.

[FNB] F. Faure, S. Nonnenmacher, and S. De Bièvre, Scarred eigenstates for quantum cat maps of minimal periods. Comm. Math. Phys. 239 (2003), no. 3, 449-492.

[FP] M. Feingold and A. Peres, Distribution of matrix elements of chaotic systems. Phys. Rev. A (3) 34 (1986), no. 1, 591-595.

[GL] P. Gérard and E. Leichtnam, Ergodic properties of eigenfunctions for the Dirichlet problem, Duke Math J. 71 (1993), 559-607.

[GSj] A. Grigis and J. Sjöstrand, Microlocal analysis for differential operators, London Math. Soc. Lecture Notes 196 (1994).

[GS1] V. Guillemin and M. Stenzel, Grauert tubes and the homogeneous MongeAmpère equation. J. Differential Geom. 34 (1991), no. 2, 561-570.

[GS2] V. Guillemin and M. Stenzel, Grauert tubes and the homogeneous MongeAmpère equation. II. J. Differential Geom. 35 (1992), no. 3, 627-641.

[GSt] V. Guillemin and S. Sternberg, Geometric asymptotics. Mathematical Surveys, No. 14. American Mathematical Society, Providence, R.I., (1977).

[Gut] B. Gutkin, Note on converse quantum ergodicity. Proc. Amer. Math. Soc. 137 (2009), no. 8, 2795-2800.

[HanL] Q. Han and F.H. Lin Nodal sets of solutions of elliptic differential equations, book in preparation (2007).

[Has] A. Hassell, Ergodic billiards that are not quantum unique ergodic, Appendix by A. Hassell and L. Hillairet, to appear in Ann. Math. (arXiv:0807.0666).

[HZ] A. Hassell and S. Zelditch, Quantum ergodicity of boundary values of eigenfunctions. Comm. Math. Phys. 248 (2004), no. 1, 119-168. 
[HR] D. A. Hejhal and B. N. Rackner, On the topography of Maass waveforms for PSL(2, Z). Experiment. Math. 1 (1992), no. 4, 275-305.

[Hel] S. Helgason, Topics in harmonic analysis on homogeneous spaces. Progress in Mathematics, 13. Birkhuser, Boston, Mass., (1981).

[He] E. J. Heller, Bound-state eigenfunctions of classically chaotic Hamiltonian systems: scars of periodic orbits. Phys. Rev. Lett. 53 (1984), no. 16, 1515-1518.

[He2] E. J. Heller, Wavepacket dynamics and quantum chaology. Chaos et physique quantique (Les Houches, 1989), 547-664, North-Holland, Amsterdam, (1991).

[HO] E. J. Heller and P. W. O'Connor, Quantum localization for a strongly classical chaotic system, Phys. Rev. Lett. 61 (20) (1988), 2288-2291.

[Hez] H. Hezari, Complex zeros of eigenfunctions of 1D Schrdinger operators. Int. Math. Res. Not. IMRN 2008, no. 3, Art. ID rnm148, 23 pp.

[Hol] R. Holowinsky, Sieving for mass equidistribution, to appear in Annals of Math. (arXiv:0809.1640).

[HS] R. Holowinsky and K. Soundararajan, Mass equidistribution for Hecke eigenforms, to appear in Annals of Math. (arXiv:0809.1636).

[HoI-IV] L. Hörmander, Theory of Linear Partial Differential Operators I-IV, SpringerVerlag, New York (1985).

[J] O. Jenkinson, Every ergodic measure is uniquely maximizing, Disc. Cont. Dyn. Syst. 16 (2006).

[Kl] W. Klingenberg, Lectures on Closed Geodesics, Grundlehren der. math. W. 230, Springer-Verlag (1978).

[KR] P. Kurlberg and Z. Rudnick, On the distribution of matrix elements for the quantum cat map. Ann. of Math. (2) 161 (2005), no. 1, 489-507.

[L] V. F. Lazutkin, KAM theory and semiclassical approximations to eigenfunctions. With an addendum by A. I. Shnirelman. Ergebnisse der Mathematik und ihrer Grenzgebiete (3), 24. Springer-Verlag, Berlin, (1993).

[LemS1] L. Lempert and R. Szöke, Global solutions of the homogeneous complex MongeAmpère equation and complex structures on the tangent bundle of Riemannian manifolds. Math. Ann. 290 (1991), no. 4, 689-712.

[Lin] F.H. Lin, Nodal sets of solutions of elliptic and parabolic equations. Comm. Pure Appl. Math. 44 (1991), no. 3, 287-308.

[LIND] E. Lindenstrauss, Invariant measures and arithmetic quantum ergodicity, Annals of Math. (2) 163 (2006), no. 1, 165-219.

[LIND2] E. Lindenstrauss, Adelic dynamics and arithmetic quantum unique ergodicity. Current developments in mathematics, 2004, 111-139, Int. Press, Somerville, MA, (2006).

[LS] W. Luo and P. Sarnak, Quantum ergodicity of eigenfunctions on $\mathrm{PSL}_{2}(\mathbb{Z}) \backslash \mathbf{H}^{2}$, IHES Publ. 81 (1995), 207-237.

[LS2] W. Luo and P. Sarnak, Quantum variance for Hecke eigenforms, Annales Scient. de l'École Norm. Sup. 37 (2004), p. 769-799.

[M] J. Marklof, Arithmetic quantum chaos, Encyclopedia of Mathematical Physics article 449.

[MOZ] J. Marklof and S. O'Keefe, Weyl's law and quantum ergodicity for maps with divided phase space. With an appendix "Converse quantum ergodicity" by Steve Zelditch. Nonlinearity 18 (2005), no. 1, 277-304.

[MS] S. D. Miller and W. Schmid, The highly oscillatory behavior of automorphic distributions for SL(2). Lett. Math. Phys. 69 (2004), 265-286.

[NJT] N. Nadirashvili, D. Jakobson, and J.A. Toth, Geometric properties of eigenfunctions. (Russian) Uspekhi Mat. Nauk 56 (2001), no. 6(342), 67-88; translation in Russian Math. Surveys 56 (2001), no. 6, 1085-1105.

[NV] S. Nonnenmacher and A. Voros, Eigenstate structures around a hyperbolic point. J. Phys. A 30 (1997), no. 1, 295-315. 
[NV2] S. Nonnenmacher and A. Voros, Chaotic eigenfunctions in phase space. J. Statist. Phys. 92 (1998), no. 3-4, 431-518.

[NZ] N. Nonnenmacher and M. Zworski, Quantum decay rates in chaotic scattering, to appear in Acta. Math. ( arXiv:0706.3242).

[O] J. P. Otal, Sur les fonctions propres du laplacien du disque hyperbolique. (French. English, French summary) [About eigenfunctions of the Laplacian on the hyperbolic disc] C. R. Acad. Sci. Paris Sér. I Math. 327 (1998), no. 2, 161-166.

[Ra] J. Ralston, Gaussian beams and the propagation of singularities. Studies in partial differential equations, 206-248, MAA Stud. Math., 23, Math. Assoc. America, Washington, DC, (1982).

[Ra2] J. Ralston, Approximate eigenfunctions of the Laplacian. J. Differential Geometry 12 (1977), no. 1, 87-100.

[Riv] G. Riviere, Entropy of semiclassical measures in dimension 2 (arXiv: 0809.0230).

[RS] Z. Rudnick and P. Sarnak, The behaviour of eigenstates of arithmetic hyperbolic manifolds. Comm. Math. Phys. 161 (1994), no. 1, 195-213.

[Ru] D. Ruelle, Statistical mechanics: Rigorous results. W. A. Benjamin, Inc., New York-Amsterdam (1969).

[Sar] P. Sarnak, Integrals of products of eigenfunctions. Internat. Math. Res. Notices no. 6 (1997), 251-261.

[Sar2] P. Sarnak, Arithmetic quantum chaos. The Schur lectures (1992) (Tel Aviv), 183-236, Israel Math. Conf. Proc., 8, Bar-Ilan Univ., Ramat Gan, (1995). 1926.

[Sar3] P. Sarnak, Recent progress on QUE, BAMS (to appear).

[Sch] W. Schmid, Automorphic distributions for SL $(2, \mathbb{R})$. (English summary) Confrence Mosh Flato 1999, Vol. I (Dijon), 345-387, Math. Phys. Stud., 21, Kluwer Acad. Publ., Dordrecht, (2000).

[Schu] R. Schubert, On the rate of quantum ergodicity for quantised maps. Ann. Henri Poincaré 9 (2008), no. 8, 1455-1477.

[Schu2] R. Schubert, Upper bounds on the rate of quantum ergodicity. Ann. Henri Poincaré 7 (2006), no. 6, 1085-1098.

[Schu3] R. Schubert, Semiclassical behaviour of expectation values in time evolved Lagrangian states for large times. Comm. Math. Phys. 256 (2005), no. 1, 239-254.

[Sh.1] A. I. Shnirelman, Ergodic properties of eigenfunctions, Usp. Math. Nauk. 29/6 (1974), 181-182.

[Sh.2] A. I. Shnirelman, On the asymptotic properties of eigenfunctions in the region of chaotic motion, addendum to V.F.Lazutkin, KAM theory and semiclassical approximations to eigenfunctions, Springer (1993).

[SZ] B. Shiffman and S. Zelditch, Distribution of zeros of random and quantum chaotic sections of positive line bundles. (English summary) Comm. Math. Phys. 200 (1999), no. 3, 661-683.

[Sound1] K. Soundararajan, Quantum unique ergodicity for $S L_{2}(Z) \mathbf{H}$, to appear in Annals of Math. (arXiv:0901.4060)

[Su] T. Sunada, Quantum ergodicity. Progress in inverse spectral geometry, 175-196, Trends Math., Birkhuser, Basel, 1997.

[TZ1] J. A. Toth and S. Zelditch, Counting nodal lines which touch the boundary of an analytic domain. J. Differential Geom. 81 (2009), no. 3, 649-686.

[TZ2] J. A. Toth and S. Zelditch, $L^{p}$ norms of eigenfunctions in the completely integrable case. Ann. Henri Poincaré 4 (2003), no. 2, 343-368.

[TZ3] J. A. Toth and S. Zelditch, Quantum ergodic restriction theorems, I: interior hypersurfaces in domains with ergodic billiards (preprint, 2010).

[Wat] T. Watson, Central Value of Rankin Triple L-function for Unramified Maass Cusp Forms, to appear in Annals of Math. 
[W] S. A. Wolpert, Semiclassical limits for the hyperbolic plane. Duke Math. J. 108 (2001), no. 3, 449-509.

[W2] S. A. Wolpert, Asymptotic relations among Fourier coefficients of automorphic eigenfunctions. Trans. Amer. Math. Soc. 356 (2004), no. 2, 427-456.

[W3] S. A. Wolpert, The modulus of continuity for $\Gamma_{0}(m) \backslash \mathbb{H}$ semi-classical limits, Comm. Math. Phys. 216 (2001), 313-323.

[Y1] S. T. Yau, Survey on partial differential equations in differential geometry. Seminar on Differential Geometry, pp. 3-71, Ann. of Math. Stud., 102, Princeton Univ. Press, Princeton, N.J., (1982).

[Z1] S. Zelditch, Local and global analysis of eigenfunctions, in: Handbook of Geometric Analysis, No. 1 L. Ji, P. Li, R. Schoen and L. Simon (eds.), Somerville, MA: International Press; Beijing: Higher Education Press. Advanced Lectures in Mathematics (ALM) 7, 545-658 (2008).

[Z2] S. Zelditch, Quantum ergodicity and mixing, Encyclopedia of Mathematical Physics Vol. 4, Ed. J.P. Franoise, G. Naber, S.T. Tsou (2007), 183-196.

[Z3] S. Zelditch, Uniform distribution of eigenfunctions on compact hyperbolic surfaces. Duke Math. J. 55 (1987), no. 4, 919-941.

[Z4] S. Zelditch, On the rate of quantum ergodicity. I. Upper bounds. Comm. Math. Phys. 160 (1994), no. 1, 81-92.

[Z5] S. Zelditch, Complex zeros of real ergodic eigenfunctions. Invent. Math. 167 (2007), no. 2, 419-443.

[Z6] S. Zelditch, Quantum ergodicity of $C^{*}$ dynamical systems. Comm. Math. Phys. 177 (1996), no. 2, 507-528.

[Z7] S. Zelditch, Note on quantum unique ergodicity, Proc. Amer. Math. Soc. 132 (2004), no. 6, 1869-1872.

[Z8] S. Zelditch, Mean Lindelöf hypothesis and equidistribution of cusp forms and Eisenstein series. J. Funct. Anal. 97 (1991), no. 1, 1-49.

[Z9] S. Zelditch, Quantum transition amplitudes for ergodic and for completely integrable systems. J. Funct. Anal. 94 (1990), no. 2, 415-436.

[Z10] S. Zelditch, On a "quantum chaos" theorem of R. Schrader and M. Taylor. J. Funct. Anal. 109 (1992), no. 1, 1-21.

[ZZw] S.Zelditch and M.Zworski, Ergodicity of eigenfunctions for ergodic billiards, Comm.Math. Phys. 175 (1996), 673-682.

[Zh] P. Zhao, Quantum Variance of Maass-Hecke Cusp Forms, to appear in Comm. Math. Phys.

Department of Mathematics, Johns Hopkins University, Baltimore, MD 21218, USA

E-mail address: zel@math.jhu.edu 